\title{
Stability crossing set for systems with three scalar delay channels
}

\author{
Keqin Gu $\cdot$ Xie Zheng
}

Received: 19 October 2013 / Revised: 7 March 2014 / Accepted: 20 March 2014 / Published online: 11 April 2014

(C) Springer-Verlag Berlin Heidelberg 2014

\begin{abstract}
The characteristic function of a system with three scalar delay channels contains cross terms of different delays. This article studies the parameterization and geometric structure of the stability crossing set (the set of delay combinations with at least one characteristic root on the imaginary axis) for such systems. Understanding the structure of this set is crucial to the identification of stable regions in the delay parameter space using the D-subdivision method. The presence of the cross terms significantly complicates the analysis, and requires a quite different method than the case without these cross terms, and it involves more numerical computation.
\end{abstract}

Keywords Time delay - Differential-difference equations . Stability · Quasipolynomial

\section{Introduction}

This article studies the stability problem of time-delay systems with characteristic quasipolynomial

$$
\begin{aligned}
\Delta(s)= & p_{0}(s)+p_{1}(s) \mathrm{e}^{-\tau_{1} s}+p_{2}(s) \mathrm{e}^{-\tau_{2} s}+p_{3}(s) \mathrm{e}^{-\tau_{3} s} \\
& +p_{12}(s) \mathrm{e}^{-\left(\tau_{1}+\tau_{2}\right) s}+p_{23}(s) \mathrm{e}^{-\left(\tau_{2}+\tau_{3}\right) s} \\
& +p_{31}(s) \mathrm{e}^{-\left(\tau_{3}+\tau_{1}\right) s}+p_{123}(s) \mathrm{e}^{-\left(\tau_{1}+\tau_{2}+\tau_{3}\right) s}
\end{aligned}
$$

Submitted to the Special Issue on Numerical Method in Time-Delay Systems, International Journal of Dynamics and Control.

K. Gu $(\bowtie) \cdot X$. Zheng

Department of Mechanical and Industrial Engineering, Southern Illinois University Edwardsville, Edwardsville, IL 62026-1805, USA

e-mail:kgu@siue.edu

$X$. Zheng

e-mail: xzheng@siue.edu where $p_{0}(s), p_{k}(s), p_{k l}(s), k, l=1,2,3$ and $p_{123}(s)$ are polynomials of $s$ with real coefficients. It is know that the system is of retarded type if the order of $p_{0}(s)$ is higher than all the other coefficient polynomials. If at least one of them have the same order as $p_{0}(s)$, then the system is of neutral type. If the order $p_{0}(s)$ is lower than at least one of the other ceofficient polynomials, then the system is always unstable for any positive delays.

The objective of this article is to identify the set of delay parameters $\left(\tau_{1}, \tau_{2}, \tau_{3}\right)$ such that the system is stable. The main focus is to parameterize and geometrically characterize the stability crossing set, which is defined as the set of delay combinations $\left(\tau_{1}, \tau_{2}, \tau_{3}\right)$ such that the characteristic equation has at least one imaginary solution. The importance of the stability crossing set lies in the following fact: For a large class of systems, the roots of the characteristic equation

$\Delta(s)=0$

in a neighborhood of the imaginary axis and on the right half plane are continuous functions of the delays $\left(\tau_{1}, \tau_{2}, \tau_{3}\right)$. Therefore the stability crossing set divides the threedimensional delay parameter space into regions such that the number of right-half-plane roots is fixed in each such region. By studying the direction of crossing of roots at the imaginary axis, the number of right-half-plane roots in each such region can be determined, and therefore, the stable parameter regions can also be determined as a special case.

The stability analysis based on the above principle is known as the D-subdivision method (also known as the D-decomposition or D-partition method). The readers are referred to the survey paper [1] by Gryazina, et al., for a comprehensive review on this subject. D-subdivision method is especially important for time-delay systems as direct stability analysis using, for example, the Pontryagin's Theorem [2] 
is very difficult to carry out in practice. The $\mathrm{D}$-subdivision method for time-delay systems was developed by Neimark [3], and the English coverage of the method can be found in the book [4] by El'sgol'ts and Norkin. A rich collection of D-subdivision analysis in the form of stability charts can be found in the book [5] by Stépán, which also considers systems with distributed delays.

The validity of D-subdivision method requires the continuity of characteristic roots in a neighborhood of the imaginary axis and on the right half plane. This is automatically satisfied for systems of retarded type [6]. However, this is not necessarily the case for systems of neutral type with multiple delays. Such discontinuity is intimately related to the behavior of the difference equations of continuous time, as documented in [7] and [8]. Such discontinuity has been responsible for such surprising phenomena as the wellposedness or stability at small delays $[9,10]$, instability of Smith predictor due to delay mismatch [11], and sensitivity to delay variations in the discrete implementation of distributed delay feedback control [12,13]. A review and unified discussion of these phenomena can be found in [14].

In practice, the application of the $\mathrm{D}$-subdivision method is not trivial when it is used to analyze systems with multiple delays. See, for example, [15]. In [16], Hale and Huang give a complete analysis of the first order differential-difference equation with two delays, and all the stable delay parameter regions are obtained. In the last decade or so, many analyses have been conducted on systems with two or three delays with various generality. Examples include systems with two delays without cross term [17], multiple delay systems with cross terms [18], polynomial eigenvalue approach [19], and three variable delays with additional fixed delays [20].

The main focus of this article is to obtain explicit parameterization and geometric characterization of stability crossing set of the system given in (1.1). These have been achieved for systems with two or three delays without cross terms in [21] and [22], respectively, and for systems with two delays with a cross term in [23]. The case discussed here is complicated by the presence of the cross terms such as $\mathrm{e}^{-\left(\tau_{1}+\tau_{2}\right) s}$ and $\mathrm{e}^{-\left(\tau_{1}+\tau_{2}+\tau_{3}\right) s}$ involving the sums of delays in the exponents. As will be seen later, such a system requires a very different method that involves more numerical computation than the cases mentioned above, although some ideas developed in [23] are instrumental.

Similar to the two delay case, the importance of including the cross terms can be described as follows: As argued in [24] and implied in [9], a linear system that contains three single-input-single-output delay elements may be described in a feedback configuration such that the forward subsystem does not contain any delay,

$$
\begin{aligned}
& \dot{x}(t)=A x(t)+B u(t), \\
& y(t)=C x(t)+D u(t),
\end{aligned}
$$

where

$$
\begin{aligned}
& x(t) \in \mathbb{R}^{n}, \\
& u(t)=\left(\begin{array}{l}
u_{1}(t) \\
u_{2}(t) \\
u_{3}(t)
\end{array}\right) \in \mathbb{R}^{3}, \\
& y(t)=\left(\begin{array}{l}
y_{1}(t) \\
y_{2}(t) \\
y_{3}(t)
\end{array}\right) \in \mathbb{R}^{3},
\end{aligned}
$$

and the feedback subsystem consists of three scalar delay elements

$$
\left(\begin{array}{l}
u_{1}(t) \\
u_{2}(t) \\
u_{3}(t)
\end{array}\right)=\left(\begin{array}{l}
y_{1}\left(t-\tau_{1}\right) \\
y_{2}\left(t-\tau_{2}\right) \\
y_{3}\left(t-\tau_{3}\right)
\end{array}\right)
$$

In other words, such a system can be considered as having three scalar delay channels. After substituting (1.3) and (1.4) by (1.5), the characteristic equation of the system can be easily seen as

$\Delta(s)=\operatorname{det}\left(\begin{array}{c|c}s I-A & -B E\left(\tau_{1}, \tau_{2}, \tau_{3}\right) \\ ---+-------- \\ -C & I-D E\left(\tau_{1}, \tau_{2}, \tau_{3}\right)\end{array}\right)=0$,

where

$E\left(\tau_{1}, \tau_{2}, \tau_{3}\right)=\operatorname{diag}\left(\mathrm{e}^{-\tau_{1} s}\left|\mathrm{e}^{-\tau_{2} s}\right| \mathrm{e}^{-\tau_{3} s}\right)$.

An application of the Schur determinant complement yields

$$
\begin{aligned}
\Delta(s) & =\operatorname{det}\left(\begin{array}{c|c}
s I-A & -B E\left(\tau_{1}, \tau_{2}, \tau_{3}\right) \\
---+ & ------ \\
0 & M(s)
\end{array}\right) \\
& =\operatorname{det}(s I-A) \cdot \operatorname{det}[M(s)],
\end{aligned}
$$

where

$M(s)=I-\left(D+C(s I-A)^{-1} B\right) E\left(\tau_{1}, \tau_{2}, \tau_{3}\right)$.

An expansion of $\operatorname{det}[M(s)]$ shows that $\Delta(s)$ indeed has the form (1.1) in general.

The remaining part of this article is organized as follows. Section 2 presents the problem setting and nontriviality assumptions. Section 3 presents local parameterization of stability crossing set using the frequency $\omega$ and a delay $\tau_{w}$. Section 4 defines the valid parameter range of $\left(\omega, \tau_{w}\right)$ and makes some nondegeneracy assumptions, and presents some implications of these assumptions. Section 5 discusses the boundary of the valid parameter range and the classification of each connected piece of this boundary. Section 6 discusses constant frequency curves, which are subsets of the stability crossing set corresponding to a fixed $\omega$ and a maximal 
$\tau_{w}$-interval. Section 7 discusses the calculation of the crossing frequency set $\Omega$ and its partition to maximal intervals $\Omega_{i}^{h}$ of different classes. Section 8 refines the classification of $\Omega_{i}^{h}$. Section 9 presents the parameterization and geometric characterization of the crossing frequency set corresponding to each maximal interval $\Omega_{i}^{h}$ of all possible types except one. This remaing type is presented in Sect. 10. Section 11 presents an example of stability analysis using the theory developed. Section 12 presents some concluding remarks.

\section{Problem setup}

Some notation used in this article is defined below. $\mathbb{R}, \mathbb{R}^{n}$, and $\mathbb{R}^{n \times m}$ denote the sets of real scalars, $n$-dimensional real vectors, and $(n \times m)$-dimensional real matrices, respectively. Similarly, $\mathbb{C}, \mathbb{C}^{n}$ and $\mathbb{C}^{n \times m}$ denote the corresponding sets with complex entries. For an $\alpha \in \mathbb{C}, \alpha^{*}$ represents its complex conjugage, and $\operatorname{Re}(\alpha)$ and $\operatorname{Im}(\alpha)$ represent its real part and imaginary part, respectively. $\mathbb{R}_{+}$represents the set of nonnegative real scalars, and $\mathbb{R}_{+}^{n}$ is the set of $n$-dimensional vectors with components in $\mathbb{R}_{+}$. $\mathbb{C}_{+}$and $\mathbb{C}_{-}$denote the sets of complex numbers with nonnegative real parts and strictly negative real parts, respectively. For a given set $\mathcal{S}, \mathcal{S}^{o}$ represents its interior and $\overline{\mathcal{S}}$ represents its closure. For example, $\mathbb{R}_{+}^{o}$ represents the set of strictly positive real numbers. We also define the set of integers as $\mathbb{Z}$, and $\mathbb{Z}_{3}^{+}=\{1,2,3\}$.

For an infinite number of objects indexed by one integer, $Q_{k}, k \in \mathbb{Z}$, we say there are $a$ series of such objects. Typically, these objects approximately line up along one direction in the three-dimensional space. We say there are an array of objects to refer to objects indexed by two integers, $Q_{k l}$, $k \in \mathbb{Z}, l \in \mathbb{Z}$, and they typically line up approximately along two directions. We say there are a lattice of objects when referring to objects indexed by three integers, $Q_{k l m}, k \in \mathbb{Z}$, $l \in \mathbb{Z}, m \in \mathbb{Z}$ and they typically line up approximately in three directions.

We will also freely exchange the order of the subscripts of the polynomials in $\Delta(s)$. For example, $p_{123}$ may also be written as $p_{321}$ or $p_{231}$, and $p_{12}$ may also be written as $p_{21}$. Therefore, for arbitrary $\{u, v, w\}=\mathbb{Z}_{3}^{+}$, we may write (1.1) as

$$
\begin{aligned}
\Delta(s)= & p_{0}(s)+p_{u}(s) \mathrm{e}^{-\tau_{u} s}+p_{v}(s) \mathrm{e}^{-\tau_{v} s}+p_{w}(s) \mathrm{e}^{-\tau_{w} s} \\
& +p_{u v}(s) \mathrm{e}^{-\left(\tau_{u}+\tau_{v}\right) s}+p_{v w}(s) \mathrm{e}^{-\left(\tau_{v}+\tau_{w}\right) s} \\
& +p_{w u}(s) \mathrm{e}^{-\left(\tau_{w}+\tau_{u}\right) s} \\
& +p_{u v w}(s) \mathrm{e}^{-\left(\tau_{u}+\tau_{v}+\tau_{w}\right) s} .
\end{aligned}
$$

The three delays $\left(\tau_{1}, \tau_{2}, \tau_{3}\right)$ may be considered as a point in the 3-dimensional coordinate system with coordinate unit vectors $\boldsymbol{\tau}_{1}^{0}, \boldsymbol{\tau}_{2}^{0}$ and $\boldsymbol{\tau}_{3}^{0}$, and the point may be written as a vector

$\boldsymbol{\tau}=\tau_{u} \boldsymbol{\tau}_{u}^{0}+\tau_{v} \boldsymbol{\tau}_{v}^{0}+\tau_{w} \boldsymbol{\tau}_{w}^{0}$.
As $\Delta$ depends on both $s$ and $\tau_{1}, \tau_{2}$ and $\tau_{3}$,we may sometimes denote it as $\Delta_{\tau}(s)$ or $\Delta_{\tau_{1}, \tau_{2}, \tau_{3}}(s)$ when we want to consider $\Delta$ as a function of $s$ for the given delays, and write $\Delta(s, \tau)$ or $\Delta\left(s, \tau_{1}, \tau_{2}, \tau_{3}\right)$ when we want to emphasize it as a function of both $s$ and the delays. Similar notation is also used for other functions of $s$ and $\boldsymbol{\tau}$.

For the sake of convenience, we say a system is "stable" to mean it is asymptotically stable. We will restrict ourselves to systems that satisfy the following non-triviality assumptions:

\section{Assumption I Existence of principal term}

$\operatorname{ord}\left(p_{0}\right) \geq \operatorname{ord}\left(p_{u}\right)$, for all $u \in \mathbb{Z}_{3}^{+}$;

$\operatorname{ord}\left(p_{0}\right) \geq \operatorname{ord}\left(p_{u v}\right)$, for all $u \in \mathbb{Z}_{3}^{+}, v \in \mathbb{Z}_{3}^{+}, u \neq v$;

$\operatorname{ord}\left(p_{0}\right) \geq \operatorname{ord}\left(p_{123}\right)$,

where $\operatorname{ord}(\cdot)$ denotes the order of the polynomial.

\section{Assumption II Zero frequency restriction}

$$
\begin{aligned}
& p_{0}(0)+p_{1}(0)+p_{2}(0)+p_{3}(0) \\
& \quad+p_{12}(0)+p_{23}(0)+p_{31}(0)+p_{123}(0) \neq 0
\end{aligned}
$$

\section{Assumption III Infinite-frequency restriction}

$$
\begin{aligned}
1 & >\lim _{s \rightarrow \infty} \frac{1}{\left|p_{0}(s)\right|}\left[\left|p_{1}(s)\right|+\left|p_{2}(s)\right|+\left|p_{3}(s)\right|\right. \\
& \left.+\left|p_{12}(s)\right|+\left|p_{23}(s)\right|+\left|p_{31}(s)\right|+\left|p_{123}(s)\right|\right] .
\end{aligned}
$$

The above assumptions are made to make sure the problem considered is meaningful, and are similar in spirit to the ones made in [21,22] and [23]. If Assumption I is violated, then it is well known that the system is unstable for arbitrary positive delays [2,6]. If Assumption II is violated, then 0 is a characteristic root for arbitrary delays, and therefore the system cannot be stable for any delays. Assumption III is automatically satisfied if the system is of retarded type. For system of neutral type, Assumption III is a simple condition to guarantee that the difference equation associated with this system is stable for arbitrary positive delays. Less restrictive conditions to guarantee the stability of the difference equations for arbitrary positive delays may be used instead of Assumption III. Such a condition also guarentees the continuity of the characteristic roots in the neighborhood of the imaginary axis and the right half plane. Interested readers are referred to [7,14,25-27] for more extended discussions on this issue.

The main focus of this study is the stability crossing set, which is defined as follows.

Definition 2.1 The stability crossing set, denoted as $\mathcal{T}$, is the set of all $\left(\tau_{1}, \tau_{2}, \tau_{3}\right) \in \mathbb{R}_{+}^{3}$ such that $\Delta_{\tau_{1}, \tau_{2}, \tau_{3}}(s)=0$ has at least one solution on the imaginary axis. An $\omega>0$ is known as a crossing frequency if there exists a delay combination $\left(\tau_{1}, \tau_{2}, \tau_{3}\right) \in \mathbb{R}_{+}^{3}$ such that

$\Delta\left(j \omega, \tau_{1}, \tau_{2}, \tau_{3}\right)=0$. 
The set of all such $\omega$ is known as the crossing frequency set, and is denoted as $\Omega$. For any given set $\Gamma \subset \mathbb{R}_{+}^{o}$, we define $\mathcal{T}_{\Gamma}$ as the set of $\left(\tau_{1}, \tau_{2}, \tau_{3}\right) \in \mathbb{R}^{3}$ such that (2.2) is satisfied for some $\omega \in \Gamma$. Especially, when $\Gamma$ is a singleton $\{\omega\}$, we may write $\mathcal{T}_{\omega}$ instead of $\mathcal{T}_{\{\omega\}}$.

Note that it is not necessary to consider $\omega<0$ due to the fact that (2.2) is satisfied if and only if

$\Delta\left(-j \omega, \tau_{1}, \tau_{2}, \tau_{3}\right)=0$.

Assumption II also excludes the possibility of $\omega=0$ as a crossing frequency. Therefore, it is sufficient to restrict $\Omega \subset$ $\mathbb{R}_{+}^{o}$. Obviously,

$\mathcal{T}_{\Gamma} \neq \varnothing$

if and only if

$\Omega \cap \Gamma \neq \varnothing$.

Noted also that while $\mathcal{T}$ is restricted to $\mathbb{R}_{+}^{3}, \mathcal{T}_{\Gamma}$ and $\mathcal{T}_{\omega}$ are not. As is well known [6], the system may not be stable if $\left(\tau_{1}, \tau_{2}, \tau_{3}\right) \notin \mathbb{R}_{+}^{3}$, and therefore, $\mathcal{T}$ is of practical meaning only in $\mathbb{R}_{+}^{3}$. On the other hand, the solutions to (2.2) for a given $\omega$ have some repetitive pattern that is more conveniently described if no such restriction is imposed. With this convention in mind, we have the following relation

$\mathcal{T}=\mathcal{T}_{\Omega} \bigcap \mathbb{R}_{+}^{3}=\bigcup_{\omega \in \Omega} \mathcal{T}_{\omega} \bigcap \mathbb{R}_{+}^{3}$

For a given $w \in \mathbb{Z}_{3}^{+}$, we may use $\omega$ and $\tau_{w}$ to parameterize $\mathcal{T}$. For $u$ and $v$ such that $\{u, v, w\}=\mathbb{Z}_{3}^{+}$, such a parameterization means finding all the pairs $\left(\tau_{u}, \tau_{v}\right)$ such that $(2.2)$ is satisfied.

Definition 2.2 For a given $w \in \mathbb{Z}_{3}^{+}$, and a parametric pair $\left(\omega, \tau_{w}\right) \in \mathbb{R}_{+}^{o} \times \mathbb{R}$

$$
\mathcal{T}_{\omega, \tau_{w}}^{(w)}=\left\{\left(\tau_{1}, \tau_{2}, \tau_{3}\right) \in \mathbb{R}^{3}\right.
$$

| (2.2) is satisfied for the given $\tau_{w}$ and $\left.\omega\right\}$.

A pair $\left(\omega, \tau_{w}\right)$ is known as a valid parametric pair if $\mathcal{T}_{\omega, \tau_{w}}^{(w)} \neq$ $\varnothing$. The set of all valid parametric pair is known as the valid parameter range, and is denoted as $\Lambda^{(w)}$.

It should be noted that the crossing frequency set $\Omega$ is independent of which delay $\tau_{w}$ is used with $\omega$ to parameterize $\mathcal{T}$. On the other hand, a different choice of $w$ in general gives a different valid parameter range $\Lambda^{(w)}$.

\section{Reduced form and associated function}

For any $\{u, v, w\}=\mathbb{Z}_{3}^{+}$, we may write $\Delta(s)$ in the following $w$-reduced form

$$
\begin{aligned}
\Delta(s, \boldsymbol{\tau})= & p_{0}^{(w)}(s)+p_{u}^{(w)}(s) \mathrm{e}^{-\tau_{u} s}+p_{v}^{(w)}(s) \mathrm{e}^{-\tau_{v} s} \\
& +p_{u v}^{(w)}(s) \mathrm{e}^{-\left(\tau_{u}+\tau_{v}\right) s},
\end{aligned}
$$

where

$p_{0}^{(w)}(s)=p_{0}(s)+p_{w}(s) \mathrm{e}^{-\tau_{w} s}$,

$p_{u}^{(w)}(s)=p_{u}(s)+p_{w u}(s) \mathrm{e}^{-\tau_{w} s}$,

$p_{v}^{(w)}(s)=p_{v}(s)+p_{w v}(s) \mathrm{e}^{-\tau_{w} s}$,

$p_{u v}^{(w)}(s)=p_{u v}(s)+p_{w u v}(s) \mathrm{e}^{-\tau_{w} s}$.

Obviously, the functions defined in (3.2) to (3.5) depend on $\tau_{w}$. Similar to the case of $\Delta(s)$, such dependence may be expressed explicitly. For example, we may write $p_{u, \tau_{w}}^{(w)}(s)$ or $p_{u}^{(w)}\left(s, \tau_{w}\right)$ instead of $p_{u}^{(w)}(s)$. It is also instrumental to define the following $w$-associated function

$\Delta^{(w)}(s)=P_{0}^{(w)}(s)+P_{u}^{(w)}(s) \mathrm{e}^{-\tau_{u} s}+P_{v}^{(w)}(s) \mathrm{e}^{-\tau_{v} s}$,

where

$P_{0}^{(w)}(s)=p_{u v}^{(w)}(s) p_{u v}^{(w)}(-s)-p_{0}^{(w)}(s) p_{0}^{(w)}(-s)$,
$P_{u}^{(w)}(s)=p_{u v}^{(w)}(s) p_{v}^{(w)}(-s)-p_{u}^{(w)}(s) p_{0}^{(w)}(-s)$,
$P_{v}^{(w)}(s)=p_{u v}^{(w)}(s) p_{u}^{(w)}(-s)-p_{v}^{(w)}(s) p_{0}^{(w)}(-s)$.

A useful fact that can be easily verified by direct calculation is

$$
\begin{aligned}
\left|P_{v}^{(w)}(j \omega)\right|^{2}-\left|P_{u}^{(w)}(j \omega)\right|^{2}= & P_{0}^{(w)}(j \omega)\left[\left|p_{u}^{(w)}(j \omega)\right|^{2}\right. \\
& \left.-\left|p_{v}^{(w)}(j \omega)\right|^{2}\right]
\end{aligned}
$$

Note that there is no cross term with exponent involving $\tau_{u}+\tau_{v}$ in $\Delta_{w}(s)$. Similar to the case discussed in [23], the equation

$\Delta^{(w)}\left(j \omega, \tau_{1}, \tau_{2}, \tau_{3}\right)=0$

is closely related to the Eq. (2.2) with $\Delta(s)$ written in the $w$ reduced form (3.1). A significant advantage of working with $\Delta^{(w)}(s)$ is the fact that the three terms of $\Delta^{(w)}(j \omega)$ may be viewed as three vectors with lengths $\left|P_{0}^{(w)}(j \omega)\right|,\left|P_{u}^{(w)}(j \omega)\right|$ and $\left|P_{v}^{(w)}(j \omega)\right|$, respectively, in the complex plane. This fact is instrumental in the proof of the folloiwng proposition.

Proposition 3.1 If $\omega$ and $\tau_{w}$ is such that

$P_{0}^{(w)}(j \omega) \neq 0$

then (2.2) is satisfied if and only if (3.11) is satisfied. Under the condition (3.12), there exists a pair $\left(\tau_{u}, \tau_{v}\right)$ to satisfy (2.2) if and only if 


$$
\begin{aligned}
2\left|P_{u}^{(w)}(j \omega)\right| \geq & \mid P_{0}^{(w)}(j \omega)-\left[\left|p_{u}^{(w)}(j \omega)\right|^{2}\right. \\
& \left.-\left|p_{v}^{(w)}(j \omega)\right|^{2}\right] \mid
\end{aligned}
$$

or equivalently

$$
\begin{aligned}
2\left|P_{v}^{(w)}(j \omega)\right| \geq & \mid P_{0}^{(w)}(j \omega)+\left[\left|p_{u}^{(w)}(j \omega)\right|^{2}\right. \\
& \left.-\left|p_{v}^{(w)}(j \omega)\right|^{2}\right] \mid
\end{aligned}
$$

Furthermore, the solutions to (2.2) can be expressed as

$$
\begin{gathered}
\tau_{u}=\tau_{u}^{k_{u} \pm}\left(\omega, \tau_{w}\right)=\frac{\angle P_{u}^{(w)}(j \omega) \pm \theta_{u}+\left(2 k_{u}-1\right) \pi}{\omega}, \\
k_{u}=0, \pm 1, \pm 2, \ldots ; \\
\tau_{v}=\tau_{v}^{k_{v} \pm}\left(\omega, \tau_{w}\right)=\frac{\angle P_{v}^{(w)}(j \omega) \mp \theta_{v}+\left(2 k_{v}-1\right) \pi}{\omega} \\
k_{v}=0, \pm 1, \pm 2, \ldots ;
\end{gathered}
$$

where

$\theta_{u}=\arccos \left(\frac{P_{0}^{(w)}(j \omega)-\left[\left|p_{u}^{(w)}(j \omega)\right|^{2}-\left|p_{v}^{(w)}(j \omega)\right|^{2}\right]}{2\left|P_{u}^{(w)}(j \omega)\right|}\right)$,

$\theta_{v}=\arccos \left(\frac{P_{0}^{(w)}(j \omega)+\left[\left|p_{u}^{(w)}(j \omega)\right|^{2}-\left|p_{v}^{(w)}(j \omega)\right|^{2}\right]}{2\left|P_{v}^{(w)}(j \omega)\right|}\right)$.

The proof may be carried out in a very similar manner to those for Theorem 10, Proposition 5, and Proposition 15 in [23]. A more unified proof is given below.

Proof Comparing (3.1) and (3.6), it can be verified that

$$
\begin{aligned}
\Delta^{(w)}(j \omega)= & p_{u v}^{(w)}(j \omega) \mathrm{e}^{-j \omega\left(\tau_{u}+\tau_{v}\right)} \Delta(-j \omega) \\
& -p_{0}^{(w)}(-j \omega) \Delta(j \omega) .
\end{aligned}
$$

As (2.2) implies

$\Delta^{*}(j \omega)=\Delta(-j \omega)=0$,

it is easily seen from (3.19) that (2.2) implies (3.11). On the other hand, if (3.11) is satisfied, then

$\Delta^{(w) *}(j \omega)=\Delta^{(w)}(-j \omega)=0$,

and therefore,

$$
\begin{aligned}
P_{0}^{(w)}(j \omega) \Delta(j \omega)= & p_{0}^{(w)}(j \omega) \Delta^{(w)}(j \omega) \\
& +p_{u v}^{(w)}(j \omega) \mathrm{e}^{-j \omega\left(\tau_{u}+\tau_{v}\right)} \Delta^{(w)}(-j \omega)=0,
\end{aligned}
$$

which implies (2.2) under the condition (3.12). This proves the equivalence of (2.2) and (3.11) under the condition (3.12).

Next, we will establish the equivalence of (3.13) and (3.14). This can be done by taking square on the both sides of (3.13) and then adding

$4\left(\left|P_{v}^{(w)}(j \omega)\right|^{2}-\left|P_{u}^{(w)}(j \omega)\right|^{2}\right)$

to both sides of the resulting inequality, and using (3.10). The result can be easily seen as equivalent to taking square on both sides of (3.14).

The next step is to show that (3.13) or (3.14) is necessary and sufficient for the existence of solution for (3.11) (and equivalently (2.2) when (3.12) is satisfied). The three terms of $\Delta^{(w)}(j \omega)$ in (3.6) may be viewed as three vectors in the complex plane. The Eq. (3.11) means that these three vectors must form a triangle when arranged from head to tail. For $\omega>0$, the two vectors represented by the last two terms can assume arbitrary orientations with appropriate choice of $\tau_{u}$ and $\tau_{v}$. Therefore, there exist $\tau_{u}$ and $\tau_{v}$ to satisfy (3.11) if and only if the lengths of these vectors may form a triangle, i.e.,

$$
\begin{aligned}
& \left|P_{u}^{(w)}(j \omega)\right|+\left|P_{v}^{(w)}(j \omega)\right| \geq\left|P_{0}^{(w)}(j \omega)\right|, \\
& \left|P_{0}^{(w)}(j \omega)\right|+\left|P_{v}^{(w)}(j \omega)\right| \geq\left|P_{u}^{(w)}(j \omega)\right|, \\
& \left|P_{0}^{(w)}(j \omega)\right|+\left|P_{u}^{(w)}(j \omega)\right| \geq\left|P_{v}^{(w)}(j \omega)\right| .
\end{aligned}
$$

The first two conditions (3.20) and (3.21) together may be equivalently written as

$\left|P_{v}^{(w)}(j \omega)\right| \geq|| P_{0}^{(w)}(j \omega)|-| P_{u}^{(w)}(j \omega) \|$.

Taking square of both sides and moving terms, we arrive at the following equivalent inequality

$$
\begin{aligned}
& 2\left|P_{0}^{(w)}(j \omega)\right|\left|P_{u}^{(w)}(j \omega)\right| \\
& \geq\left|P_{0}^{(w)}(j \omega)\right|^{2}+\left|P_{u}^{(w)}(j \omega)\right|^{2}-\left|P_{v}^{(w)}(j \omega)\right|^{2} .
\end{aligned}
$$

Divising $\left|P_{0}^{(w)}(j \omega)\right|$ on both sides of the above inequality and using (3.10), we obtain

$$
\begin{aligned}
& 2\left|P_{u}^{(w)}(j \omega)\right| \geq \operatorname{sgn}\left(P_{0}^{(w)}(j \omega)\right) \\
& \quad \times\left[P_{0}^{(w)}(j \omega)-\left(\left|p_{u}^{(w)}(j \omega)\right|^{2}-\left|p_{v}^{(w)}(j \omega)\right|^{2}\right)\right] .
\end{aligned}
$$

Therefore, (3.13) implies the first two inequalites (3.20) and (3.21). Furthermore, If the third inequality (3.22) is satisfied, then the right hand side of (3.23), and therefore the right hand side of (3.24), is nonnegative. Therefore, (3.20), (3.21) 
Fig. $1 \theta_{u}$ and $\theta_{v}$ are interior angles of the triangle when $P_{0}^{(w)}>0$

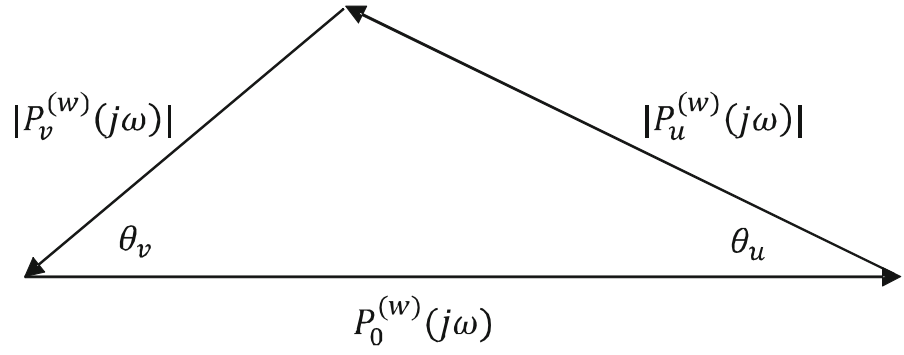

Fig. $2 \theta_{u}$ and $\theta_{v}$ are exterior angles of the triangle when $P_{0}^{(w)}>0$

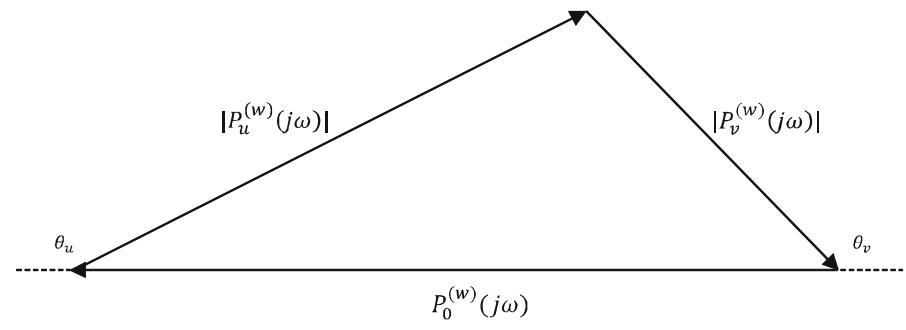

We will see in the next section that the restriction (3.12) may be removed when the system is nondegenerate.

\section{Parameter range and nondegeneracy}

When using $\omega$ and $\tau_{w}$ to parameterize $\mathcal{T}$, let the $\omega$-axis be horizontal, and $\tau_{w}$-axis be vertical. In this parametric space, the condition (3.13) or (3.14) may be used to determine the valid parameter range $\Lambda^{(w)}$, which consists of regions in $\mathbb{R}_{+}^{o} \times \mathbb{R}$ that completely parameterize $\mathcal{T}$. To be definite, we will use (3.13). Define

$$
\begin{aligned}
\phi^{(w)}\left(\omega, \tau_{w}\right)= & 4\left|P_{u}^{(w)}(j \omega)\right|^{2}-\mid P_{0}^{(w)}(j \omega) \\
& -\left.\left[\left|p_{u}^{(w)}(j \omega)\right|^{2}-\left|p_{v}^{(w)}(j \omega)\right|^{2}\right]\right|^{2} .
\end{aligned}
$$

Then, Proposition 3.1 suggests that $\left(\omega, \tau_{w}\right) \in \Lambda^{(w)}$ if and only if $\omega>0, \tau_{w} \in \mathbb{R}$ and

$\phi^{(w)}\left(\omega, \tau_{w}\right) \geq 0$.

In other words,

$\Lambda^{(w)}=\left\{\left(\omega, \tau_{w}\right) \in \mathbb{R}_{+}^{o} \times \mathbb{R} \mid \phi^{(w)}\left(\omega, \tau_{w}\right) \geq 0\right\}$.

However, the conditions in Proposition 3.1 excludes those $\left(\omega, \tau_{w}\right)$ that satisfy

$P_{0}^{(w)}\left(\omega, \tau_{w}\right)=0$.

A parametric pair $\left(\omega, \tau_{w}\right) \in \mathbb{R}_{+}^{o} \times \mathbb{R}$ that satisfies (4.4) is known as a singular parametric pair. The set of all singular parametric pairs is known as the singular parametric set and is denoted as $\Lambda_{s}^{(w)}$. We may not conclude (4.3) until we can be
Using (3.10) in the above two expressions and cancelling out $\left|P_{0}^{(w)}(j \omega)\right|$ in the numerators and the denominators, we arrive at the expressions (3.17) and (3.18). Thus the proof is complete. 
certain that it is also true on $\Lambda_{s}^{(w)}$. The following proposition does that.

Proposition 4.1 A parametric pair $\left(\omega, \tau_{w}\right) \in \mathbb{R}_{+}^{o} \times \mathbb{R}$ satisfies $\left(\omega, \tau_{w}\right) \in \Lambda^{(w)}$ if and only if it satisfies (3.13).

Proof Using (3.1), we may write

$\Delta(j \omega)=p_{0}^{(u w)}(j \omega)+p_{v}^{(u w)}(j \omega) \mathrm{e}^{-j \omega \tau_{v}}$,

where

$p_{0}^{(u w)}(j \omega)=p_{0}^{(w)}(j \omega)+p_{u}^{(w)}(j \omega) \mathrm{e}^{-j \omega \tau_{u}}$,

$p_{v}^{(u w)}(j \omega)=p_{v}^{(w)}(j \omega)+p_{u v}^{(w)}(j \omega) \mathrm{e}^{-j \omega \tau_{u}}$.

Therefore, it is obvious that (2.2) can be satisfied for some $\tau_{u}$ if and only if

$\left|p_{0}^{(u w)}(j \omega)\right|=\left|p_{v}^{(u w)}(j \omega)\right|$.

The above equation is equivalent to

$$
\begin{gathered}
\left(p_{0}^{(w)}(j \omega)+p_{u}^{(w)}(j \omega) \mathrm{e}^{-j \omega \tau_{u}}\right) \\
\cdot\left(p_{0}^{(w) *}(j \omega)+p_{u}^{(w) *}(j \omega) \mathrm{e}^{j \omega \tau_{u}}\right) \\
=\left(p_{v}^{(w)}(j \omega)+p_{u v}^{(w)}(j \omega) \mathrm{e}^{-j \omega \tau_{u}}\right) \\
\quad\left(p_{v}^{(w) *}(j \omega)+p_{u v}^{(w) *}(j \omega) \mathrm{e}^{j \omega \tau_{u}}\right) .
\end{gathered}
$$

Direct calculation indicates that the above is equivalent to

$$
\begin{aligned}
& 2\left|P_{u}^{(w)}(j \omega)\right| \cos \left(\alpha-\omega \tau_{u}\right) \\
& =\left|p_{u}^{(w)}(j \omega)\right|^{2}-\left|p_{v}^{(w)}(j \omega)\right|^{2}-P_{0}^{(w)}(j \omega),
\end{aligned}
$$

where

$\alpha=\angle P_{u}^{(w)}(j \omega)$

Obviously, (4.7) can be true for some $\tau_{u}$ if and only if (3.13) is satisfied.

Before proceeding to extend the remaining parts of Proposition 3.1 to the entire $\Lambda^{(w)}$ and further clarifying the properties of $\Lambda^{(w)}$, we introduce the following nondegeneracy assumptions.

Assumption IV Nondegeneracy of coefficients. For any $\{u, v, w\}=\mathbb{Z}_{3}^{+}$, no $\omega$ and $\tau_{w}$ may simultaneously satisfy

$P_{u}^{(w)}\left(j \omega, \tau_{w}\right)=0$,

$\left|P_{0}^{(w)}\left(j \omega, \tau_{w}\right)\right|=\left|P_{v}^{(w)}\left(j \omega, \tau_{w}\right)\right|$.
Assumption V No multiple critical delays. For a given $\omega$, if

$\phi^{(w)}\left(\omega, \tau_{w}\right)=0$,

$\frac{\partial \phi^{(w)}\left(\omega, \tau_{w}\right)}{\partial \tau_{w}}=0$

are satisfied for $\tau_{w}=\tau^{a}$ and $\tau^{b}$, then $\omega\left(\tau^{b}-\tau^{a}\right) /(2 \pi)$ must be an integer.

Assumption VI Transversality at critical freqencies. Any $\omega$ and $\tau_{w}$ that satisfy (4.10) and (4.11) must satisfy

$\frac{\partial \phi^{(w)}\left(\omega, \tau_{w}\right)}{\partial \omega} \neq 0$,
$\frac{\partial^{2} \phi^{(w)}\left(\omega, \tau_{w}\right)}{\partial \tau_{w}^{2}} \neq 0$.

Assumption VII Noncritical zero frequency. For $\omega=0$, no $\tau_{w}$ may simultaneously satisfy (4.10) and (4.11).

The above four nondegeneracy assumptions are made to reduce the number of cases to be considered while covering "almost all" cases in the sense that they are "generic" in the terminology of dynamical systems [28]. This allows us to present the main idea in a systematic way within a reasonable amount of space. Similar to Remark 3.2 in [21], cases that violate some nondegeneracy assumptions are not difficult to treat individually. The systems discussed in the rest of this article are assumed to satisfy the three nontriviality assumptions (Assumptions I to III) given in Sect. 2 and the four nondegeneracy assumptions (Assumptions IV to VII) given above.

Proposition 4.2 The valid parameter range $\Lambda^{(w)}$ has a nonempty interior that can be expressed as

$\Lambda^{(w) o}=\left\{\left(\omega, \tau_{w}\right) \in \mathbb{R}_{+}^{o} \times \mathbb{R} \mid \phi^{(w)}\left(\omega, \tau_{w}\right)>0\right\}$,

and its boundary $\partial \Lambda^{(w)}$ away from the $\tau_{w}$-axis can be characterized by

$$
\begin{aligned}
& \partial \Lambda^{(w)} \bigcap \mathbb{R}_{+}^{o} \times \mathbb{R} \\
& \quad=\left\{\left(\omega, \tau_{w}\right) \in \mathbb{R}_{+}^{o} \times \mathbb{R} \mid \phi^{(w)}\left(\omega, \tau_{w}\right)=0\right\} .
\end{aligned}
$$

Proof The Assumptions VI implies that for any $\left(\omega, \tau_{w}\right)$ that satisfies (4.10) must satisfy either (4.12) or

$\frac{\partial \phi^{(w)}\left(\omega, \tau_{w}\right)}{\partial \tau_{w}} \neq 0$,

from which the conclusions are obvious.

Proposition 4.3 For any $\left(\omega, \tau_{w}\right) \in \Lambda^{(w)}$,

$$
P_{u}^{(w)}(j \omega) \neq 0,
$$$$
P_{v}^{(w)}(j \omega) \neq 0 .
$$ 
Proof If some $\left(\omega, \tau_{w}\right) \in \Lambda^{(w)}$ violates (4.16), i.e., it satisfies (4.8), then the right hand side of the inequality (3.13) must vanish, therefore

$P_{0}^{(w)}(j \omega)=\left|p_{u}^{(w)}(j \omega)\right|^{2}-\left|p_{v}^{(w)}(j \omega)\right|^{2}$.

Multiplying both sides of the above equation by $P_{0}^{(w)}(j \omega)$ and using (3.10) and (4.8) yield

$$
\begin{aligned}
\left|P_{0}^{(w)}(j \omega)\right|^{2} & =\left|P_{v}^{(w)}(j \omega)\right|^{2}-\left|P_{u}^{(w)}(j \omega)\right|^{2} \\
& =\left|P_{v}^{(w)}(j \omega)\right|^{2} .
\end{aligned}
$$

But this violates Assumption IV. This proves (4.16). The proof of (4.17) is similar except that we use the equivalent characterization (3.14) for $\Lambda^{(w)}$ instead of (3.13), and interchange $u$ and $v$ in Assumption IV.

Proposition 4.4 For any given $\left(\omega, \tau_{w}\right) \in \Lambda^{(w)}$, all $\tau_{u}$ and $\tau_{v}$ that satisfy (2.2) can be expressed by (3.15) and (3.16).

Proof The proof is done by continuity argument. In the proof of Proposition 4.1, all the possible $\tau_{u}$ that satisfy (4.7) can be expressed as

$\tau_{u}=\frac{\alpha \pm \beta+2 k_{1} \pi}{\omega}, k_{1}=0, \pm 1, \pm 2, \ldots$,

where

$\beta=\arccos \frac{\left|p_{u}^{(w)}(j \omega)\right|^{2}-\left|p_{v}^{(w)}(j \omega)\right|^{2}-P_{0}^{(w)}(j \omega)}{2\left|P_{u}^{(w)}(j \omega)\right|}$.

Any such $\tau_{u}$ achieves (4.6). If

$\left|p_{v}^{(u w)}(j \omega)\right| \neq 0$,

then we see from (4.5) that all $\tau_{v}$ that satisfy (2.2) can be expressed as

$\tau_{v}=\frac{\angle p_{v}^{(u w)}(j \omega)-\angle p_{0}^{(u w)}(j \omega)+\left(2 k_{2}+1\right) \pi}{\omega}$,
$k_{2}=0, \pm 1, \pm 2, \ldots$

We will show that (4.19) is indeed true by contradiction. Assume the opposite, i.e.,

$p_{v}^{(u w)}(j \omega)=p_{v}^{(w)}(j \omega)+p_{u v}^{(w)}(j \omega) \mathrm{e}^{-j \omega \tau_{u}}=0$.

Then according to (4.6), we must also have

$p_{0}^{(v w)}(-j \omega)=p_{0}^{(w)}(-j \omega)+p_{u}^{(w)}(-j \omega) \mathrm{e}^{j \omega \tau_{u}}=0$.
Multiplying (4.21) by $p_{0}^{(w)}(-j \omega)$, and multiplying (4.22) by $p_{u v}^{(w)}(j \omega) \mathrm{e}^{-j \omega \tau_{u}}$, and subtracting the resulting equations, we obtain

$p_{u v}^{(w)}(j \omega) p_{u}^{(w)}(-j \omega)-p_{v}^{(w)}(j \omega) p_{0}^{(w)}(-j \omega)=0$.

The left hand side of (4.23) equals to $P_{v}^{(w)}(j \omega)$. Therefore, (4.23) is not possible according to Proposition 4.3. Thus we have shown that (4.18) and (4.20) indeed represent all the solutions to (2.2).

Next, we need to show that for $\left(\omega, \tau_{w}\right) \in \Lambda^{(w)}$, the set of $\left(\tau_{u}, \tau_{v}\right)$ expressed by (4.18) and (4.20) must be identical to the set expressed by (3.15) and (3.16). For $\left(\omega, \tau_{w}\right) \in$ $\Lambda^{(w)} \backslash \Lambda_{s}^{(w)}$, this is true as both represent all the solutions to (2.2). Because (4.18) and (4.20) are continuous functions of $\omega$ and $\tau_{w}$ for fixed $k_{1}, k_{2}$, for any given $\left(\omega, \tau_{w}\right) \in \Lambda_{s}^{(w)}$, we may find a sequence $\left(\omega_{k}, \tau_{w k}\right) \in \Lambda^{(w)} \backslash \Lambda_{s}^{(w)}, k=1,2,3, \ldots$ such that $\left(\omega_{k}, \tau_{w k}\right) \rightarrow\left(\omega, \tau_{w}\right)$ as $k \rightarrow \infty$. Then, all $\left(\tau_{u}, \tau_{v}\right)$ that correspond to $\left(\omega, \tau_{w}\right)$ given in (4.18) and (4.20) may alternatively be expressed as the limit of the sequence formed by (4.18) and (4.20) with $\left(\omega, \tau_{w}\right)$ replaced by $\left(\omega_{k}, \tau_{w k}\right)$. This sequence is equal to the one formed by (3.15) and (3.16) with $\left(\omega, \tau_{w}\right)$ replaced by $\left(\omega_{k}, \tau_{w k}\right)$. The continuity of (3.15) and (3.16) allows us to conclude that the set of $\left(\tau_{u}, \tau_{v}\right)$ given by (3.15) and (3.16) is identical to the set given by (4.18) and (4.20) for all $\left(\omega, \tau_{w}\right) \in \Lambda_{s}^{(w)}$.

It is interesting to point out that for $\left(\omega, \tau_{w}\right) \in \Lambda_{s}^{(w)}$, the Eq. (3.11) has additional solutions that cannot be expressed by (3.15) and (3.16). In other words, although the solutions (3.15) and (3.16) were derived through the associated function $\Delta^{(w)}(j \omega, \boldsymbol{\tau})$, they represent all the solutions to the original characteristic equation $\Delta(j \omega, \tau)=0$, but not $\Delta^{(w)}(j \omega, \boldsymbol{\tau})=0$ if $\omega \in \Lambda_{s}^{(w)}$. We will not discuss this point further in this article, and interested readers are referred to [23].

\section{Boundaries of parameter range}

As indicated in Proposition 4.2, the boundary of the valid parameter range away from the $\tau_{w}$-axis can be characterized by

$\phi^{(w)}\left(\omega, \tau_{w}\right)=0$.

In other words, any $\left(\omega, \tau_{w}\right) \in \partial \Lambda^{(w)} \cap\left(\mathbb{R}_{+}^{o} \times \mathbb{R}\right)$ must satisfy one of the following two equations

$$
\begin{aligned}
& 2\left|P_{u}^{(w)}(j \omega)\right| \\
& =P_{0}^{(w)}(j \omega)-\left[\left|p_{u}^{(w)}(j \omega)\right|^{2}-\left|p_{v}^{(w)}(j \omega)\right|^{2}\right], \text { or }
\end{aligned}
$$




$$
\begin{aligned}
& -2\left|P_{u}^{(w)}(j \omega)\right| \\
& =P_{0}^{(w)}(j \omega)-\left[\left|p_{u}^{(w)}(j \omega)\right|^{2}-\left|p_{v}^{(w)}(j \omega)\right|^{2}\right] .
\end{aligned}
$$

It is impossible to satisfy both (5.1) and (5.2) simultaneously in view of Proposition 4.3. Using a similar method to show the equivalence between (3.13) and (3.14) in Proposition 3.1, we can easily show that (3.13) and (3.14) with " $\geq$ " replaced by "=" are also equivalent. Therefore such an $\left(\omega, \tau_{w}\right)$ must also satisfy one and only one of the following two equations

$$
\begin{aligned}
& 2\left|P_{v}^{(w)}(j \omega)\right| \\
& =P_{0}^{(w)}(j \omega)+\left[\left|p_{u}^{(w)}(j \omega)\right|^{2}-\left|p_{v}^{(w)}(j \omega)\right|^{2}\right], \text { or } \\
& -2\left|P_{v}^{(w)}(j \omega)\right| \\
& =P_{0}^{(w)}(j \omega)+\left[\left|p_{u}^{(w)}(j \omega)\right|^{2}-\left|p_{v}^{(w)}(j \omega)\right|^{2}\right] .
\end{aligned}
$$

These allow us to divide the boundary points into four different types. The following proposition reduces the conditions from two equatoins to one equation for each type.

Proposition 5.1 Each parametric pair in the boundary of valid parameter range $\left(\omega, \tau_{w}\right) \in \partial \Lambda^{(w)} \cap\left(\mathbb{R}_{+}^{o} \times \mathbb{R}\right)$ must belong to one and only one of the following four types:

$$
\begin{array}{ll}
\text { Type 1 : } & \frac{\left|p_{v}^{(w)}\left(\omega, \tau_{w}\right)\right|^{2}-\left|p_{u}^{(w)}\left(\omega, \tau_{w}\right)\right|^{2}}{\left|P_{u}^{(w)}\left(\omega, \tau_{w}\right)\right|+\left|P_{v}^{(w)}\left(\omega, \tau_{w}\right)\right|}=-1, \\
\text { Type 2 : } & \frac{\left|p_{v}^{(w)}\left(\omega, \tau_{w}\right)\right|^{2}-\left|p_{u}^{(w)}\left(\omega, \tau_{w}\right)\right|^{2}}{\left|P_{u}^{(w)}\left(\omega, \tau_{w}\right)\right|+\left|P_{v}^{(w)}\left(\omega, \tau_{w}\right)\right|}=1, \\
\text { Type 3 : } & \frac{P_{0}^{(w)}\left(\omega, \tau_{w}\right)}{\left|P_{u}^{(w)}\left(\omega, \tau_{w}\right)\right|+\left|P_{v}^{(w)}\left(\omega, \tau_{w}\right)\right|}=-1, \\
\text { Type 4 : } & \frac{P_{0}^{(w)}\left(\omega, \tau_{w}\right)}{\left|P_{u}^{(w)}\left(\omega, \tau_{w}\right)\right|+\left|P_{v}^{(w)}\left(\omega, \tau_{w}\right)\right|}=1 .
\end{array}
$$

The corresponding $\theta_{u}$ and $\theta_{v}$ calculated by (3.17) and (3.18) are

Type 1: $\theta_{u}=0, \theta_{v}=\pi$,

Type 2: $\theta_{u}=\pi, \theta_{v}=0$,

Type 3: $\theta_{u}=\pi, \theta_{v}=\pi$,

Type 4: $\theta_{u}=0, \theta_{v}=0$.

It is worth noting that for a given $\left(\omega, \tau_{w}\right) \in \partial \Lambda^{(w)} \cap$ $\left(\mathbb{R}_{+}^{o} \times \mathbb{R}\right)$, type 1 satisfies (5.1) and (5.4), type 2 satisfies (5.2) and (5.3), type 3 satisfies (5.2) and (5.4), and type 4 satisfies (5.1) and (5.3).
Proof An $\left(\omega, \tau_{w}\right) \in \partial \Lambda^{(w)} \cap\left(\mathbb{R}_{+}^{o} \times \mathbb{R}\right)$ must satisfy either (5.1) or (5.2) but not both, and either (5.3) or (5.4) but not both. If (5.1) and (5.4) are satisfied, we may subtract (5.4) from (5.1) and divide the resulting equation by $\left|P_{u}^{(w)}\left(\omega, \tau_{w}\right)\right|+\left|P_{v}^{(w)}\left(\omega, \tau_{w}\right)\right|$ to arrive at (5.5). We may also apply the relations (5.1) and (5.4) in (3.17) and (3.18) to obtain (5.9). The proof for other types are similar.

The remaining fact to be proven is that no $\left(\omega, \tau_{w}\right) \in$ $\partial \Lambda^{(w)} \cap\left(\mathbb{R}_{+}^{o} \times \mathbb{R}\right)$ may satisfy two Eqs. in (5.5) to (5.8) simultaneously. It is obvious that (5.5) contradicts (5.6), and (5.7) contradicts (5.8). Next we will show that (5.5) and (5.7) may not be satisfied simultaneously by contradiction. If (5.5) and (5.7) are both satisfied, then multiplying these two equations and using (3.10) yield

$$
\frac{\left|P_{u}^{(w)}\left(\omega, \tau_{w}\right)\right|^{2}-\left|P_{v}^{(w)}\left(\omega, \tau_{w}\right)\right|^{2}}{\left(\left|P_{u}^{(w)}\left(\omega, \tau_{w}\right)\right|+\left|P_{v}^{(w)}\left(\omega, \tau_{w}\right)\right|\right)^{2}}=1 .
$$

Cancelling out the factor $\left|P_{u}^{(w)}\left(\omega, \tau_{w}\right)\right|+\left|P_{v}^{(w)}\left(\omega, \tau_{w}\right)\right|$ results in

$\frac{\left|P_{u}^{(w)}\left(\omega, \tau_{w}\right)\right|-\left|P_{v}^{(w)}\left(\omega, \tau_{w}\right)\right|}{\left|P_{u}^{(w)}\left(\omega, \tau_{w}\right)\right|+\left|P_{v}^{(w)}\left(\omega, \tau_{w}\right)\right|}=1$.

But the above equation requires

$P_{v}^{(w)}\left(\omega, \tau_{w}\right)=0$,

which contradicts Proposition 4.3. The proof for the remaining possible combinations (5.5) and (5.8), (5.6) and (5.7), (5.6) and (5.8) are similar.

Corollary 5.2 The type of all the parametric pairs $\left(\omega, \tau_{w}\right)$ on a continuous curve of $\partial \Lambda^{(w)} \cap\left(\mathbb{R}_{+}^{o} \times \mathbb{R}\right)$ must be identical.

Proof The proof of Proposition 5.1 made it clear that it is impossible to continuously change from one type to another.

Example 5.3 Consider a system with the characteristic quasipolynomial $\Delta(s)$ in the form of (1.1) with

$$
\begin{aligned}
& p_{0}(s)=s^{6}+3 s^{5}+8.5 s^{4}+13.5 s^{3}+10 s^{2}+10.5 s+2.5, \\
& p_{1}(s)=s^{4}+3 s^{3}+7.5 s^{2}+10.5 s+2.5, \\
& p_{2}(s)=2 s^{4}+2 s^{3}+12 s^{2}+2 s+10, \\
& p_{3}(s)=3 s^{4}+6 s^{3}+4.5 s^{2}+6 s+1.5, \\
& p_{12}(s)=7 s^{2}+2 s+1, \quad p_{13}(s)=3 s+4, \\
& p_{23}(s)=5 s+1, \quad p_{123}(s)=1 .
\end{aligned}
$$

Using $\left(\omega, \tau_{3}\right)$ to parameterize $\mathcal{T}$, the parameter range $\Lambda^{(3)}$ is shown in Fig. 3, along with the boundary types. 
Fig. $3 \Lambda^{(3)}$ and its boundary types in Example 5.3

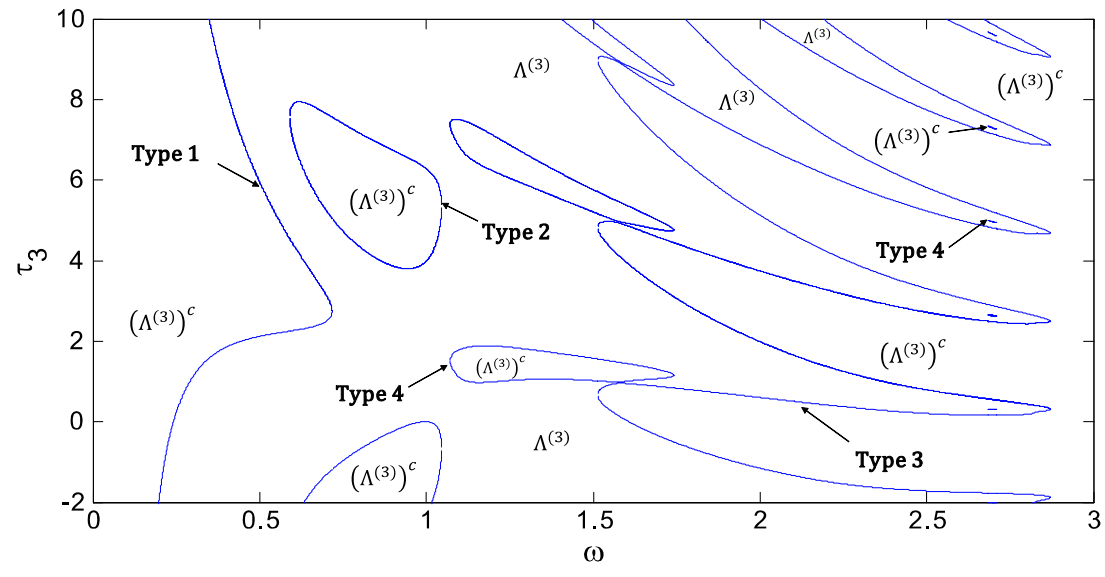

A few words is in order on the numerical process of generating the diagram in the above example. Direct calculation indicates that $\phi^{(w)}\left(\omega, \tau_{w}\right)$ may be expressed in the following form

$$
\begin{aligned}
\phi^{(w)}\left(\omega, \tau_{w}\right)= & C_{0}(\omega)+C_{1}(\omega) \mathrm{e}^{j \omega \tau_{w}}+C_{1}^{*}(\omega) \mathrm{e}^{-j \omega \tau_{w}} \\
& +C_{2}(\omega) \mathrm{e}^{j 2 \omega \tau_{w}}+C_{2}^{*}(\omega) \mathrm{e}^{-j 2 \omega \tau_{w}},
\end{aligned}
$$

where $C_{0}(\omega)$ is a real polynomial of $\omega$, and $C_{1}(\omega)$ and $C_{2}(\omega)$ are complex polynomials. Obviously, $\phi^{(w)}\left(\omega, \tau_{w}\right)$ is a periodic function of $\tau_{w}$ with period $2 \pi / \omega$.

For a fixed $\omega, \phi^{(w)}\left(\omega, \tau_{w}\right)$ is a function of $\tau_{w}$. Let

$z=\mathrm{e}^{j \omega \tau_{w}}$.

Then equation

$\phi^{(w)}\left(\omega, \tau_{w}\right)=0$

becomes a fourth order equation of $z$,

$C_{2}(\omega) z^{4}+C_{1}(\omega) z^{3}+C_{0}(\omega) z^{2}+C_{1}^{*}(\omega) z+C_{2}^{*}(\omega)=0$.

There are four solutions of $z$ to this equation. However, only those solutions on the unit circle correspond to real $\tau_{w}$ and are meaningful for our purpose. There may be either four solutions, two solutions, or no solution on the unit circle. For each solution $z$ on the unit circle, let $\tau_{w}$ satisfy (5.14), then $\tau_{w}+2 \pi k / \omega$ for any integer $k$ also satisfy (5.14).

Therefore, the diagram in the above example may be numerically generated by the following procedure: Let $\omega$ sweep through an interval $\left[0, \omega_{\max }\right]$ for a sufficiently large $\omega_{\max }$ with a sufficiently small increment. For each such $\omega$, solve (5.16) for $z$ in the unit circle and the corresponding $\tau_{w}$. This generates the boundary $\partial \Lambda^{(w)} \cap\left(\mathbb{R}_{+}^{o} \times \mathbb{R}\right)$ of the valid parameter range $\Lambda^{(w)}$. In order to judge which side of the boundary is in $\Lambda^{(w)}$, we may evaluate $\phi^{(w)}\left(\omega, \tau_{w}\right)$ at a few strategic points away from the boundary to test if it is nonnegative. Alternatively, we may test the sign of its partial derivative with respect to $\omega$ or $\tau_{w}$ at a few strategic points on the boundary. The type of each continuous piece of boundary may be obtained by testing which equation among (5.5) to (5.8) is satisfied.

Once $\Lambda^{(w)}$ is given, the stability crossing set $\mathcal{T}$ may be generated by (3.15) and (3.16) with restriction to $\mathbb{R}_{+}^{3}$. As will be seen later on, the geometric characteristics of $\mathcal{T}$ is largely determined by the structure of $\Lambda^{(w)}$ and its boundary types.

\section{Constant frequency curves}

We start this section with some concepts.

Definition 6.1 Let $\mathbf{C}: \mathbb{R} \rightarrow \mathbb{R}^{3}$, be such that

$\mathbf{C}\left(\alpha+\alpha_{0}\right)=\mathbf{C}(\alpha)+\mathbf{a}$

for some $\alpha_{0} \in \mathbb{R}_{+}^{o}$ and

$\mathbf{a}=a_{1} \boldsymbol{\tau}_{1}^{0}+a_{2} \boldsymbol{\tau}_{2}^{0}+a_{3} \boldsymbol{\tau}_{3}^{0} \in \mathbb{R}^{3}$.

Then the curve

$\mathcal{C}=\{\mathbf{C}(\alpha) \mid \alpha \in \mathbb{R}\}$

is known as a spiral, $\beta$ a for any $\beta \in \mathbb{R}, \beta \neq 0$ is known as its axis, and

$\|\mathbf{a}\|=\sqrt{a_{1}^{2}+a_{2}^{2}+a_{3}^{2}}$

is its pitch.

Obviously, a spiral is completely defined by $\mathbf{C}(\alpha)$ in an interval $\left[0, \alpha_{0}\right]$ since

$\mathcal{C}=\bigcup_{k=-\infty}^{+\infty}\left\{\mathbf{C}(\alpha)+k \mathbf{a} \mid \alpha \in\left[0, \alpha_{0}\right]\right\}$ 
Definition 6.2 For a given $\omega \in \Omega$, an interval $\left[\tau_{w}^{a}, \tau_{w}^{b}\right]$ is known as a maximal $\tau_{w}$-interval if $\left(\omega, \tau_{w}\right) \in \Lambda^{(w) o}$ for all $\tau_{w}^{a}<\tau_{w}<\tau_{w}^{b}$, and $\left(\omega, \tau_{w}^{a}\right) \in \partial \Lambda^{(w)},\left(\omega, \tau_{w}^{b}\right) \in \partial \Lambda^{(w)}$. If $\omega \in \Omega$ is such that $\left(\omega, \tau_{w}\right) \in \Lambda^{(w) o}$ for all $\tau_{w} \in \mathbb{R}$, then $(-\infty,+\infty)$ is also defined as a maximal $\tau_{w}$-interval.

Using Proposition 4.2, it can be seen that $\left[\tau_{w}^{a}, \tau_{w}^{b}\right]$ is a maximal $\tau_{w}$-interval if and only if

$\phi^{(w)}\left(\omega, \tau_{w}\right)>0$ for $\tau_{w} \in\left(\tau_{w}^{a}, \tau_{w}^{b}\right)$,

and

$\phi^{(w)}\left(\omega, \tau_{w}^{a}\right)=0, \phi^{(w)}\left(\omega, \tau_{w}^{b}\right)=0$.

If

$\phi^{(w)}\left(\omega, \tau_{w}\right)>0$ for all $\tau_{w} \in \mathbb{R}$,

then $(-\infty,+\infty)$ is the only maximal $\tau_{w}$-interval corresponding to this $\omega$.

Definition 6.3 For an $\omega \in \Omega$ and a maximal $\tau_{w}$-interval $\mathcal{I}$, the constant frequency set (corresponding to $\omega$ and $\mathcal{I}$ ) is

$\mathcal{T}_{\omega, \mathcal{I}}^{(w)}=\bigcup_{\tau_{w} \in \mathcal{I}} \mathcal{T}_{\omega, \tau_{w}}^{(w)}$

If the valid parameter range $\Lambda^{(w)}$ is plotted in a $\omega-\tau_{w}$ coordinate system as was done in Example 5.3, a maximal $\tau_{w}$-interval corresponds to a vertical line segment in $\Lambda^{(w)}$ that touches the boundary on both ends. The boundary types of the two ends determine some important geometric characteristics of the corresponding constant frequency set as is stated in the following proposition.

Proposition 6.4 Let $\omega \in \Omega$ be given, and let $\left[\tau_{w}^{a}, \tau_{w}^{b}\right]$ be a maximal $\tau_{w}$-interval, then

i) if

$\operatorname{Type}\left(\omega, \tau_{w}^{a}\right)=\operatorname{Type}\left(\omega, \tau_{w}^{b}\right)$,

then the corresponding constant frequency set consists of an array of identical closed curves;

ii) otherwise, it consists of a series of identical spirals.

In the following, we will list all the possibilities, which also serves as the proof for the above proposition. The following notation will be used below

$$
\begin{aligned}
\mathcal{T}_{\omega, \mathcal{I}}^{(w) k_{u} k_{v}+} & =\bigcup_{\tau_{w} \in \mathcal{I}}\left\{\left(\tau_{1}, \tau_{2}, \tau_{3}\right) \mid \tau_{u}=\tau_{u}^{k_{u}+}\left(\omega, \tau_{w}\right),\right. \\
\tau_{v} & \left.=\tau_{v}^{k_{v}+}\left(\omega, \tau_{w}\right)\right\},
\end{aligned}
$$

$$
\begin{aligned}
\mathcal{T}_{\omega, \mathcal{I}}^{(w) k_{u} k_{v}-} & =\bigcup_{\tau_{w} \in \mathcal{I}}\left\{\left(\tau_{1}, \tau_{2}, \tau_{3}\right) \mid \tau_{u}=\tau_{u}^{k_{u}-}\left(\omega, \tau_{w}\right),\right. \\
\tau_{v} & \left.=\tau_{v}^{k_{v}-}\left(\omega, \tau_{w}\right)\right\},
\end{aligned}
$$

where $\tau_{u}^{k_{u} \pm}\left(\omega, \tau_{w}\right)$ and $\tau_{v}^{k_{v} \pm}\left(\omega, \tau_{w}\right)$ are given in (3.15) and (3.16), respectively. It is noted that $\mathcal{T}_{\omega, \mathcal{I}}^{(w) k_{u} k_{v}+}$ for different $k_{u}$ and $k_{v}$ have an identical shape, and can be obtained from any one of them by moving a multiple of $2 \pi / \omega$ in $\boldsymbol{\tau}_{u}^{0}$ and $\boldsymbol{\tau}_{v}^{0}$ directions. The same thing can be said about $\mathcal{T}_{\omega, \mathcal{I}}^{(w) k_{u} k_{v}-}$.

Case i) Type $\left(\omega, \tau_{w}^{a}\right)=\operatorname{Type}\left(\omega, \tau_{w}^{b}\right)$ :

a. Type $\left(\omega, \tau_{w}^{a}\right)=\operatorname{Type}\left(\omega, \tau_{w}^{b}\right)=1$. Using (5.9), it is easily seen that for both $\tau_{w}=\tau_{w}^{a}$ and $\tau_{w}^{b}$,

$\tau_{u}^{k_{u}+}\left(\omega, \tau_{w}\right)=\tau_{u}^{k_{u}-}\left(\omega, \tau_{w}\right)$, $\tau_{v}^{k_{v}+}\left(\omega, \tau_{w}\right)=\tau_{v}^{k_{v}-1,-}\left(\omega, \tau_{w}\right)$.

This means that $\mathcal{T}_{\omega,\left[\tau_{w}^{u}, \tau_{w}^{b}\right]}^{(w) k_{u} k_{v}+}$ is connected with $\mathcal{T}_{\omega,\left[\tau_{w}^{a}, \tau_{w}^{b}\right]}^{(w) k_{u}, k_{v}-1,-}$ at both ends, and form a closed curve

$\mathcal{T}_{\omega,\left[\tau_{w}^{a}, \tau_{w}^{b}\right]}^{(w) k_{u} k_{v}}=\mathcal{T}_{\omega,\left[\tau_{w}^{a}, \tau_{w}^{b}\right]}^{(w) k_{u} k_{v}+} \bigcup \mathcal{T}_{\omega,\left[\tau_{w}^{a}, \tau_{w}^{b}\right]}^{(w) k_{k}, k_{v}-1,-}$.

Obviously, $\mathcal{T}_{\omega,\left[\tau_{w}^{a}, \tau_{w}^{b}\right]}^{(w) k_{v} k_{v}}$ for different $k_{u}$ and $k_{v}$ have an identical shape, and may be obtained from any one of them by moving a multiple of $2 \pi / \omega$ in $\boldsymbol{\tau}_{u}^{0}$ and $\boldsymbol{\tau}_{v}^{0}$ directions. The constant frequency set consists of an array of such closed curves

$$
\mathcal{T}_{\omega,\left[\tau_{w}^{a}, \tau_{w}^{b}\right]}^{(w)}=\bigcup_{k_{u}=-\infty}^{+\infty} \bigcup_{k_{v}=-\infty}^{+\infty} \mathcal{T}_{\omega,\left[\tau_{w}^{a}, \tau_{w}^{b}\right]}^{(w) k_{u} k_{v}}
$$

b. Type $\left(\omega, \tau_{w}^{a}\right)=\operatorname{Type}\left(\omega, \tau_{w}^{b}\right)=2$. Then,

$$
\mathcal{T}_{\omega,\left[\tau_{w}^{a}, \tau_{w}^{b}\right]}^{(w) k_{u} k_{v}}=\mathcal{T}_{\omega,\left[\tau_{w}^{a}, \tau_{w}^{b}\right]}^{(w) k_{k} k_{v}+} \bigcup \mathcal{T}_{\omega,\left[\tau_{w}^{a}, \tau_{w}^{b}\right]}^{(w) k_{u}+1, k_{v},-}
$$

is a closed curve for any $k_{u}, k_{v} \in \mathbb{Z}$. These curves are $2 \pi / \omega$ apart in both $\boldsymbol{\tau}_{u}^{0}$ and $\boldsymbol{\tau}_{v}^{0}$ directions. The constant frequency set $\mathcal{T}_{\omega,\left[\tau_{w}^{a}, \tau_{w}^{b}\right]}^{(w)}$ can again be expressed as (6.4).

c. Type $\left(\omega, \tau_{w}^{a}\right)=\operatorname{Type}\left(\omega, \tau_{w}^{b}\right)=3$. Then

$$
\mathcal{T}_{\omega,\left[\tau_{w}^{a}, \tau_{w}^{b}\right]}^{(w) k_{k} k_{v}}=\mathcal{T}_{\omega,\left[\tau_{w}^{a}, \tau_{w}^{b}\right]}^{(w) k_{k} k_{v}+} \bigcup \mathcal{T}_{\omega,\left[\tau_{w}^{a}, \tau_{w}^{b}\right]}^{(w) k_{u}+1, k_{v}-1,-}
$$

is a closed curve with the same repetition pattern.

d. Type $\left(\omega, \tau_{w}^{a}\right)=\operatorname{Type}\left(\omega, \tau_{w}^{b}\right)=4$. Then

$\mathcal{T}_{\omega,\left[\tau_{w}^{a}, \tau_{w}^{b}\right]}^{(w) k_{u} k_{v}}=\mathcal{T}_{\omega,\left[\tau_{w}^{a}, \tau_{w}^{b}\right]}^{(w) k_{k} k_{v}+} \bigcup \mathcal{T}_{\omega,\left[\tau_{w}^{a}, \tau_{w}^{b}\right]}^{(w) k_{u} k_{v}-}$

form a closed curve with the same repetition pattern. 
Case ii) $\operatorname{Type}\left(\omega, \tau_{w}^{a}\right) \neq \operatorname{Type}\left(\omega, \tau_{w}^{b}\right)$ :

a. Type $\left(\omega, \tau_{w}^{a}\right)=1$, and $\operatorname{Type}\left(\omega, \tau_{w}^{b}\right)=2$. In this case, $\mathcal{T}_{\omega,\left[\tau_{w}^{a}, \tau_{w}^{b}\right]}^{(w) k_{k}+}$ is connected with $\mathcal{T}_{\omega,\left[\tau_{w}^{a}, \tau_{w}^{b}\right]}^{(w) k_{u}, k_{v}-1,-}$ at $\tau_{w}^{a}$, which is in turn connected with $\mathcal{T}_{\omega,\left[\tau_{w}^{a}, \tau_{w}^{b}\right]}^{(w) k_{v}-1,+}$ at $\tau_{w}^{b}$, which is further connected with $\mathcal{T}_{\omega,\left[\tau_{w}^{a}, \tau_{w}^{b}\right]}^{(w) k_{u}-1, k_{v}-2,-}$ at $\tau_{w}^{a}$, and so on. This forms a spiral. Each such spiral may be expressed as

$$
\mathcal{T}_{\omega,\left[\tau_{w}^{a}, \tau_{w}^{b}\right]}^{(w) k_{u v}}=\bigcup_{k=-\infty}^{+\infty}\left(\mathcal{T}_{\omega,\left[\tau_{w}^{a}, \tau_{w}^{b}\right]}^{(w) k, k_{u v}+k,+} \cup \mathcal{T}_{\omega,\left[\tau_{w}^{a}, \tau_{w}^{b}\right]}^{(w) k, k_{k v}+k-1,-}\right)
$$

for some $k_{u v} \in \mathbb{Z}$. These spirals have an identical shape, and different spirals can be obtained from any one by moving along the $\boldsymbol{\tau}_{u}^{0}$ or $\boldsymbol{\tau}_{v}^{0}$ direction by multiples of $2 \pi / \omega$. They have a common axis

$\boldsymbol{\tau}=\boldsymbol{\tau}_{u}^{0}+\boldsymbol{\tau}_{v}^{0}$

The constant frequency set consists of a series of such spirals

$\mathcal{T}_{\omega,\left[\tau_{w}^{a}, \tau_{w}^{b}\right]}^{(w)}=\bigcup_{k_{u v}=-\infty}^{+\infty} \mathcal{T}_{\omega,\left[\tau_{w}^{a}, \tau_{w}^{b}\right]}^{(w) k_{u v}}$.

If we reverse the types, i.e., if $\left(\omega, \tau_{w}^{a}\right)$ is type 2 , and $\left(\omega, \tau_{w}^{b}\right)$ is type 1 , then $\mathcal{T}_{\omega,\left[\tau_{w}^{a}, \tau_{w}^{b}\right]}^{(w) k_{u} k_{v}+}$ is still connected with $\mathcal{T}_{\omega,\left[\tau_{w}^{a}, \tau_{w}^{b}\right]}^{(w) k_{u}, k_{v}-1,-}$, but at $\tau_{w}^{b}$ instead $\tau_{w}^{a}$, and so on. Therefore (6.8) still represents a spiral, but the connections are at the opposite ends. The remaining subcases are analogous when the type is reversed, and will not be explicitly discussed.

b. Type $\left(\omega, \tau_{w}^{a}\right)=1$, and Type $\left(\omega, \tau_{w}^{b}\right)=3$. Then $\mathcal{T}_{\omega,\left[\tau_{w}^{a}, \tau_{w}^{b}\right]}^{(w) k_{v} k_{v}+}$ is connected with $\mathcal{T}_{\omega,\left[\tau_{w}^{a}, \tau_{w}^{b}\right]}^{(w) k_{u}, k_{v}-1,-}$ at $\tau_{w}^{a}$, which is in turn is connected with $\mathcal{T}_{\omega,\left[\tau_{w}^{a}, \tau_{w}^{b}\right]}^{(w) k_{v},+}$ at $\tau_{w}^{b}$, and so on. This pattern allows us to conclude that $\mathcal{T}_{\omega,\left[\tau_{w}^{a}, \tau_{w}^{b}\right]}^{(w)}$ consists of a seris of the spirals

$$
\begin{gathered}
\mathcal{T}_{\omega,\left[\tau_{w}^{a}, \tau_{w}^{b}\right]}^{(w) k_{u v}}=\bigcup_{k=-\infty}^{+\infty}\left(\mathcal{T}_{\omega,\left[\tau_{w}^{a}, \tau_{w}^{b}\right]}^{(w) k k_{u v}+} \cup \mathcal{T}_{\omega,\left[\tau_{w}^{a}, \tau_{w}^{b}\right]}^{(w) k, k_{u v}-1,-}\right), \\
k_{u v}=0, \pm 1, \pm 2, \ldots
\end{gathered}
$$

The axis of the spirals is

$$
\tau=\tau_{u}^{0}
$$

Different spirals can be obtained from any one by moving along the $\boldsymbol{\tau}_{v}^{0}$ direction by multiples of $2 \pi / \omega$.

c. Type $\left(\omega, \tau_{w}^{a}\right)=1$, and Type $\left(\omega, \tau_{w}^{b}\right)=4$. Then $\mathcal{T}_{\omega,\left[\tau_{w}^{a}, \tau_{w}^{b}\right]}^{(w) k_{u} k_{v}+}$ is connected with $\mathcal{T}_{\omega,\left[\tau_{w}^{a}, \tau_{w}^{b}\right]}^{(w) k_{u}, k_{v}-1,-}$ at $\tau_{w}^{a}$, which is in turn connected with $\mathcal{T}_{\omega,\left[\tau_{w}^{a}, \tau_{w}^{b}\right]}^{(w) k_{u}, k_{v}-1,+}$ at $\tau_{b}$, and so on. Therefore, $\mathcal{T}_{\omega,\left[\tau_{w}^{a}, \tau_{w}^{b}\right]}^{(w)}$ consists of the spirals

$$
\begin{gathered}
\mathcal{T}_{\omega,\left[\tau_{w}^{a}, \tau_{w}^{b}\right]}^{(w) k_{u v}}=\bigcup_{k=-\infty}^{+\infty}\left(\mathcal{T}_{\omega,\left[\tau_{w}^{a v}, \tau_{w}^{b}\right]}^{(w) k_{u}, k,+} \cup \mathcal{T}_{\omega,\left[\tau_{w}^{a}, \tau_{w}^{b}\right]}^{(w) k_{u v}, k-1,-}\right), \\
k_{u v}=0, \pm 1, \pm 2, \ldots
\end{gathered}
$$

The axis of the spirals is

$$
\boldsymbol{\tau}=\boldsymbol{\tau}_{v}^{0}
$$

From one spiral, the remaining spirals can be generated by moving along the $\boldsymbol{\tau}_{u}^{0}$ direction by multiples of $2 \pi / \omega$.

d. Type $\left(\omega, \tau_{w}^{a}\right)=2$, and $\operatorname{Type}\left(\omega, \tau_{w}^{b}\right)=3$. In this case, $\mathcal{T}_{\omega,\left[\tau_{w}^{a}, \tau_{w}^{b}\right]}^{(w) k_{v}+}$ is connected with $\mathcal{T}_{\omega,\left[\tau_{w}^{a}, \tau_{w}^{b}\right]}^{(w) k_{v}+k_{v},-}$ at $\tau_{w}^{a}$, which in turn is connected with $\mathcal{T}_{\omega,\left[\tau_{w}^{a}, \tau_{w}^{b}\right]}^{(w) k_{u}, k_{v}+1,+}$ at $\tau_{w}^{b}$, and so on. Therefore, $\mathcal{T}_{\omega,\left[\tau_{w}^{a}, \tau_{w}^{b}\right]}^{(w)}$ consists of the spirals

$$
\begin{gathered}
\mathcal{T}_{\omega,\left[\tau_{w}^{u}, \tau_{w}^{b}\right]}^{(w) k_{u v}}=\bigcup_{\substack{k=-\infty \\
k_{u v}}}^{+\infty}\left(\mathcal{T}_{\omega,\left[\tau_{w}^{u}, \tau_{w}^{b}\right]}^{(w) k_{u v} k+} \cup \mathcal{T}_{\omega,\left[\tau_{w}^{a}, \tau_{w}^{b}\right]}^{(w) k_{u v}+1, k,-}\right) \\
\end{gathered}
$$

The axis of the spirals is

$\boldsymbol{\tau}=\boldsymbol{\tau}_{v}^{0}$.

From one spiral, the remaining spirals can be obtained by moving along the $\boldsymbol{\tau}_{u}^{0}$ direction by multiples of $2 \pi / \omega$.

e. Type $\left(\omega, \tau_{w}^{a}\right)=2$, and Type $\left(\omega, \tau_{w}^{b}\right)=4$. In this case, $\mathcal{T}_{\omega,\left[\tau_{w}^{a}, \tau_{w}^{b}\right]}^{(w) k_{v}+}$ is connected with $\mathcal{T}_{\omega,\left[\tau_{w}^{a}, \tau_{w}^{b}\right]}^{(w) k_{v}+k_{v},-}$ at $\tau_{w}^{a}$, which in turn is connected with $\mathcal{T}_{\omega,\left[\tau_{w}^{a}, \tau_{w}^{b}\right]}^{(w) k_{u}+1, k_{v},+}$ at $\tau_{w}^{b}$, and so on. Therefore, $\mathcal{T}_{\omega,\left[\tau_{w}^{a}, \tau_{w}^{b}\right]}^{(w)}$ consists of the spirals

$$
\begin{gathered}
\mathcal{T}_{\omega,\left[\tau_{w}^{u}, \tau_{w}^{b}\right]}^{(w) k_{k v}}=\bigcup_{\begin{array}{c}
k=-\infty \\
k_{u v}
\end{array}=0, \pm 1, \pm 2, \ldots}^{+\infty}\left(\mathcal{T}_{\omega,\left[\tau_{w}^{a}, \tau_{w}^{b}\right]}^{(w) k k_{w v}+} \cup \mathcal{T}_{\omega,\left[\tau_{w}^{a}, \tau_{w}^{b}\right]}^{(w) k+1, k_{u v},-}\right),
\end{gathered}
$$

The axis of the spirals is

$\tau=\tau_{u}^{0}$.

From one spiral, the remaining spirals can be obtained by moving along the $\boldsymbol{\tau}_{v}^{0}$ direction by multiples of $2 \pi / \omega$.

f. Type $\left(\omega, \tau_{w}^{a}\right)=3$, and Type $\left(\omega, \tau_{w}^{b}\right)=4$. In this case, $\mathcal{T}_{\omega,\left[\tau_{w}^{a}, \tau_{w}^{b}\right]}^{(w) k_{k},}$ is connected with $\mathcal{T}_{\omega,\left[\tau_{w}^{a}, \tau_{w}^{b}\right]}^{(w) k_{v}+1, k_{v}-1,-}$ at $\tau_{w}^{a}$, which in turn is connected with $\mathcal{T}_{\omega,\left[\tau_{w}^{a}, \tau_{w}^{b}\right]}^{(w) k_{v}+1,+}$ at 
$\tau_{w}^{b}$, and so on. Therefore, $\mathcal{T}_{\omega,\left[\tau_{w}^{a}, \tau_{w}^{b}\right]}^{(w)}$ consists of the spirals

$$
\begin{gathered}
\mathcal{T}_{\omega,\left[\tau_{w}^{a}, \tau_{w}^{b}\right]}^{(w) k_{u v}}=\bigcup_{k=-\infty}^{+\infty}\left(\mathcal{T}_{\omega,\left[\tau_{w}^{a}, \tau_{w}^{b}\right]}^{(w) k, k_{u v}-k,+} \cup \mathcal{T}_{\omega,\left[\tau_{w}^{a}, \tau_{w}^{b}\right]}^{(w) k+1, k_{u v}-k-1,-}\right), \\
k_{u v}=0, \pm 1, \pm 2, \ldots
\end{gathered}
$$

The axis of the spirals is

$$
\boldsymbol{\tau}=\boldsymbol{\tau}_{u}^{0}-\boldsymbol{\tau}_{v}^{0}
$$

From one spiral, the remaining spirals can be obtained by moving along the $\boldsymbol{\tau}_{u}^{0}$ or $\boldsymbol{\tau}_{v}^{0}$ direction by multiples of $2 \pi / \omega$.

We will call the closed curve $\mathcal{T}_{\omega,\left[\tau_{w}^{a}, \tau_{w}^{b}\right]}^{(w) k_{u} k_{v}}$ or the spiral $\mathcal{T}_{\omega,\left[\tau_{w}^{a}, \tau_{w}^{b}\right]}^{(w) k_{u v}}$ a constant frequency curve. Then we can say that the constant frequency set consists of an array of constant frequency curves (closed curves) in case i), and a series of constant frequency curves (spirals) in case ii) above.

It should be pointed out that if $\left[\tau_{w}^{a}, \tau_{w}^{b}\right]$ is a maximal $\tau_{w^{-}}$ interval, then $\left[\tau_{w}^{a}+k_{w} 2 \pi / \omega, \tau_{w}^{b}+k_{w} 2 \pi / \omega\right]$ for any integer $k_{w}$ is also a maximal $\tau_{w}$-interval. It can be easily seen that $\mathcal{T}_{\omega,\left[\tau_{w}^{a}+k_{w} 2 \pi / \omega, \tau_{w}^{b}+k_{w} 2 \pi / \omega\right]}^{(w) k_{u v}}$ may be obtained by moving $\mathcal{T}_{\omega,\left[\tau_{w}^{a}, \tau_{w}^{b}\right]}^{(w) k_{u v}}$ a distance of $k_{w} 2 \pi / \omega$ in the $\boldsymbol{\tau}_{w}^{0}$ direction.

For case ii) in Proposition 6.4, it is interesting to note that the spirals are more conveniently parameterized by a different delay than $\tau_{w}$. For example, in the case ii-b), the complete spiral $\mathcal{T}_{\omega,\left[\tau_{w}^{a}, \tau_{w}^{b}\right]}^{(w) k}$ may be parameterized by $\tau_{u} \in \mathbb{R}$ without the need to divide into different segments. This can be done by using a $u$-reduced form instead of $w$-reduced form in (3.1). Similarly, in the case ii-a), the spiral $\mathcal{T}_{\omega,\left[\tau_{w}^{a}, \tau_{w}^{b}\right]}^{(w)}$ may be completely parameterized by either $\tau_{u}$ or $\tau_{v}$ in the range of $(-\infty,+\infty)$, which can be accomplished by either using the $u$-reduced form or the $v$-reduced form. On the other hand, the geometric characterization in this parameterization requires considering the case with an infinitely long maximal delay interval, which will be discussed next. An important tool for this purpose is the rotation index defined below.

\section{Definition 6.5 Let}

$F\left(\tau_{w}\right)=A+B \mathrm{e}^{j \omega \tau_{w}}+C \mathrm{e}^{-j \omega \tau_{w}}$,

where $A, B, C \in \mathbb{C}$. If

$F\left(\tau_{w}\right) \neq 0$ for all $\tau_{w} \in[0,2 \pi / \omega]$,

then as $\tau_{w}$ increases from 0 to $2 \pi / \omega$, let the corresponding increment of $\angle F\left(\tau_{w}\right)$ be $\Delta \angle F\left(\tau_{w}\right)$. Then the rotation index of $F$ is defined as

$\delta_{\tau_{w}}(F)=\left\{\begin{aligned} 0 & \text { if } \Delta \angle F\left(\tau_{w}\right)=0 \\ 1 & \text { if } \Delta \angle F\left(\tau_{w}\right)=2 \pi \\ -1 & \text { if } \Delta \angle F\left(\tau_{w}\right)=-2 \pi\end{aligned}\right.$

The method to evaluate the rotation index is stated in the following proposition.

Proposition 6.6 Let $F\left(\tau_{w}\right)$ be defined as (6.15). Let

$\psi=\frac{\angle B+\angle C}{2}$.

Then,

i) If

$|B| \neq|C|$

define

$\Theta=\left[\frac{\operatorname{Re}\left(A \mathrm{e}^{-j \psi}\right)}{|B|+|C|}\right]^{2}+\left[\frac{\operatorname{Im}\left(A \mathrm{e}^{-j \psi}\right)}{|B|-|C|}\right]^{2}$.

Then the condition (6.16) is satisfied if and only if

$\Theta \neq 1$,

in which case,

$\delta_{\tau_{w}}(F)=\left\{\begin{array}{cl}0 & \text { if } \Theta>1, \\ 1 & \text { if } \Theta<1 \text { and }|B|>|C|, \\ -1 & \text { if } \Theta<1 \text { and }|B|<|C| .\end{array}\right.$

ii) On the other hand, if

$|B|=|C|$,

then the condition (6.16) is satisfied if and only if either

$\operatorname{Im}\left(A \mathrm{e}^{-j \psi}\right) \neq 0$

or

$\left|\operatorname{Re}\left(A \mathrm{e}^{-j \psi}\right)\right|>|B|+|C|$

are satisfied, in which case

$\delta_{\tau_{w}}(F)=0$. 
Proof It can be shown that

$$
\begin{aligned}
\mathrm{e}^{-j \psi} F\left(\tau_{w}\right)= & {\left[\operatorname{Re}\left(A \mathrm{e}^{-j \psi}\right)+(|B|+|C|) \cos \left(\omega \tau_{w}+\phi\right)\right] } \\
& +j\left[\operatorname{Im}\left(A \mathrm{e}^{-j \psi}\right)+(|B|-|C|) \sin \left(\omega \tau_{w}+\phi\right)\right],
\end{aligned}
$$

where

$\phi=\frac{\angle B-\angle C}{2}$.

If $|B| \neq|C|$, the Eq. (6.19) means that $F\left(\tau_{w}\right)$ traces out an ellipse as $\tau_{w}$ increases by $2 \pi / \omega$. This ellipse is centered at $A$, its major semi-axis is $(|B|+|C|)$ oriented in the $\mathrm{e}^{j \psi}$ direction, and its minor semi-axis is $|(|B|-|C|)|$ oriented in the perpendicular direction. Therefore, this ellipse passes through the origin (i.e., (6.16) is violated) if and only if $\Theta=1$. If $\Theta>1$, the origin is outside of this ellipse, and there is no net increase of $\angle F\left(\tau_{w}\right)$ as we go through this ellipse and return to the original position. If $\Theta<1$, then the origin is inside of this ellipse, in which case the rotation is counterclockwise as $\tau_{w}$ increases by $2 \pi / \omega$ if $|B|>|C|$, resulting in a net increase of $\angle F\left(\tau_{w}\right)$ by $2 \pi$; the rotation is clockwise if $|B|<|C|$, resulting in a net increase by $-2 \pi$.

If $|B|=|C|$, then $F\left(\tau_{w}\right)$ traces out a line segment with $\mathrm{e}^{j \psi}\left[A \mathrm{e}^{-j \psi} \pm(|B|+|C|)\right]$ as its two end points. The origin is on this line segment if and only if both (6.17) and (6.18) are violated. If the origin is not on the line segment, then there is no net increase of $\angle F\left(\tau_{w}\right)$ as $F\left(\tau_{w}\right)$ returns to the original value.

Using the rotation index in Definition 6.5, the constant frequency curve corresponding to a maximal $\tau_{w}$-interval of infinite length may be described below.

Proposition 6.7 Let $\omega \in \Omega$ be given such that $\left(\omega, \tau_{w}\right) \in$ $\Lambda^{(w)}$ for all $\tau_{w} \in \mathbb{R}$. Then the corresponding constant frequency set $\mathcal{T}_{\omega,(-\infty,+\infty)}^{(w)}$ can be expressed as

$\mathcal{T}_{\omega,(-\infty,+\infty)}^{(w)}=\bigcup_{k_{u}, k_{v} \text { integers }}\left(\mathcal{T}_{\omega,(-\infty,+\infty)}^{(w) k_{u} k_{v}+} \bigcup \mathcal{T}_{\omega,(-\infty,+\infty)}^{(w) k_{u} k_{v}-}\right)$

where $\mathcal{T}_{\omega,(-\infty,+\infty)}^{(w) k_{u} k_{v} \pm}$ are spirals defined as

$\mathcal{T}_{\omega,(-\infty,+\infty)}^{(w) k_{u} k_{v} \pm}=\left\{\begin{array}{l|l}\left(\tau_{1}, \tau_{2}, \tau_{3}\right) & \begin{array}{l}\tau_{w} \in(-\infty,+\infty) \\ \tau_{u}=\tau_{u}^{k_{u} \pm}\left(\omega, \tau_{w}\right) \\ \tau_{v}=\tau_{v}^{k_{v} \pm}\left(\omega, \tau_{w}\right)\end{array}\end{array}\right\}$

and $\tau_{u}^{k_{u} \pm}\left(\omega, \tau_{w}\right)$ and $\tau_{v}^{k_{v} \pm}\left(\omega, \tau_{w}\right)$ are defined in (3.15) and (3.16), respectively. Each $\mathcal{T}_{\omega,(-\infty,+\infty)}^{(w) k_{u} k_{v}+}$ or $\mathcal{T}_{\omega,(-\infty,+\infty)}^{(w) k_{u} k_{v}-}$ is a spiral with axis

$\boldsymbol{\tau}=\delta_{\tau_{w}}\left(P_{u}^{(w)}(\omega, \cdot)\right) \boldsymbol{\tau}_{u}^{0}+\delta_{\tau_{w}}\left(P_{v}^{(w)}(\omega, \cdot)\right) \boldsymbol{\tau}_{v}^{0}+\boldsymbol{\tau}_{w}^{0}$
$\mathcal{T}_{\omega,(-\infty,+\infty)}^{(w) k_{u} k_{v}+}$ is identical in shape for different $k_{u}$ and $k_{v}$. Given any one, all the others may be obtained by moving $a$ multiple of $2 \pi / \omega$ distance in the $\boldsymbol{\tau}_{u}^{0}$ direction then moving a multiple of $2 \pi / \omega$ distance in the $\tau_{v}^{0}$ direction. The same can be said about $\mathcal{T}_{\omega,(-\infty,+\infty)}^{(w) k_{u} k_{v}-}$.

Proof From Proposition 4.3, the condition (6.16) is satisfied for $F=P_{u}^{(w)}(\omega, \cdot)$ or $P_{v}^{(w)}(\omega, \cdot)$ in Definition 6.5. Therefore, $\delta_{\tau_{w}}\left(P_{u}^{(w)}(\omega, \cdot)\right)$ and $\delta_{\tau_{w}}\left(P_{v}^{(w)}(\omega, \cdot)\right)$ in (6.20) are well defined. In order to show the spirals and their axes, let $\tau_{w}$ increase $2 \pi / \omega$. Because both $P_{u}^{(w)}\left(\omega, \tau_{w}\right)$ and $P_{v}^{(w)}\left(\omega, \tau_{w}\right)$ may be written in the form of (6.15) for a given $\omega, \angle P_{u}^{(w)}\left(\omega, \tau_{w}\right)$ and $\angle P_{v}^{(w)}\left(\omega, \tau_{w}\right)$ increase $2 \pi \delta_{\tau_{w}}\left(P_{u}^{(w)}(\omega, \cdot)\right)$ and $2 \pi \delta_{\tau_{w}}\left(P_{v}^{(w)}(\omega, \cdot)\right)$, respectively. The remaing terms in (3.15) and (3.16) return to the original values. Therefore, $\tau_{u}$ and $\tau_{v}$ increase by $2 \pi \delta_{\tau_{w}}\left(P_{u}^{(w)}(\omega, \cdot)\right) / \omega$ and $2 \pi \delta_{\tau_{w}}\left(P_{v}^{(w)}(\omega, \cdot)\right) / \omega$, respectively. This shows that $\mathcal{T}_{\omega,(-\infty,+\infty)}^{(w) k_{u} k_{v} \pm}$ for each given $k_{u}, k_{v}$ and + or - may be completely determined by one segment parameterized by $\tau_{w}$ in an interval of length $2 \pi / \omega$. The other segments may be obtained by moving this segment in the direction $\tau$ given in (6.20). Therefore, they are indeed spirals with the common axis $\boldsymbol{\tau}$. The other conclusions can be seen directly from (3.15) and (3.16).

\section{Crossing frequency set and its partition}

Consider again the Eq. (5.15), or (5.16) after the variable transformation (5.14). For a fixed $\omega$, recall that there may be zero, two, or four solutions to (5.16) for $z$ on the unit circle, which corresponds to zero, two, or four real solutions to $(5.15)$ for $\tau_{w}$ in a $2 \pi / \omega$ range. When the number of such solutions change, it is necessary that

$\frac{\partial \phi^{(w)}\left(\omega, \tau_{w}\right)}{\partial \tau_{w}}=0$

Using (5.13) and (5.14), the Eq. (7.1) becomes

$2 C_{2}(\omega) z^{4}+C_{1}(\omega) z^{3}-C_{1}^{*}(\omega) z-2 C_{2}^{*}=0$.

Unlike (5.16), the structure of the Eq. (7.2) means that there are at least two solutions to (7.2) for $z$ on the unit circle for a fixed $\omega$. These two solutions correspond to the maximum and minimum of $\phi^{(w)}\left(\omega, \tau_{w}\right)$ as a function of $\tau_{w}$. When (7.2) has four solutions on the unit circle, the remaining two solutions correspond to a local maximum and a local minimum of $\phi^{(w)}\left(\omega, \tau_{w}\right)$.

For a fixed $\omega$, the four solutions to (7.2) on the unit circle correspond to four real $\tau_{w}$ within a $2 \pi / \omega$ range. We denote these solutons as $\tau_{w}^{G M}, \tau_{w}^{L M}, \tau_{w}^{L V}$ and $\tau_{w}^{G V}$ such that, 


$$
\begin{aligned}
& \phi^{(w)}\left(\omega, \tau_{w}^{G M}\right) \geq \phi^{(w)}\left(\omega, \tau_{w}^{L M}\right) \geq \phi^{(w)}\left(\omega, \tau_{w}^{L V}\right) \\
& \quad \geq \phi^{(w)}\left(\omega, \tau_{w}^{G V}\right) .
\end{aligned}
$$

Let

$M_{g}=\phi^{(w)}\left(\omega, \tau_{w}^{G M}\right)$,

$V_{g}=\phi^{(w)}\left(\omega, \tau_{w}^{G V}\right)$,

$M_{l}=\phi^{(w)}\left(\omega, \tau_{w}^{L M}\right)$,

$V_{l}=\phi^{(w)}\left(\omega, \tau_{w}^{L V}\right)$.

Then $M_{g}$ is the global maximum, $V_{g}$ is the global minimum, $M_{l}$ is the local maximum, and $V_{l}$ is the local minimum. When (7.2 ) has only two unit circle solutions, $\tau_{w}^{L M}$ and $\tau_{w}^{L V}$ do not exist. The continuity of solutions with respect to the polynomial coefficients implies that we may always find four solutions to (7.1), say, $\tau_{w 1}, \tau_{w 2}, \tau_{w 3}$ and $\tau_{w 4}$, as continuous functions of $\omega$ as long as $C_{2}(\omega) \neq 0$. In general, some of them may not be real. Even when they are all real, $\tau_{w}^{G M}$, $\tau_{w}^{L M}, \tau_{w}^{L V}$ and $\tau_{w}^{G V}$ may not be continuous functions of $\omega$ when $M_{g}, V_{g}, M_{l}$ and $V_{l}$ are not all distinct. For example, it is possible that

$\phi^{(w)}\left(\omega, \tau_{w 1}\right)=M_{g}(\omega)$,

$\phi^{(w)}\left(\omega, \tau_{w 2}\right)=M_{l}(\omega)$,

for $\omega \in\left[\omega^{*}-\varepsilon, \omega^{*}\right]$ and

$\phi^{(w)}\left(\omega, \tau_{w 2}\right)=M_{g}(\omega)$,

$\phi^{(w)}\left(\omega, \tau_{w 1}\right)=M_{l}(\omega)$,

for $\omega \in\left(\omega^{*}, \omega^{*}+\varepsilon\right]$. In this case, $\tau_{w}^{G M}$ and $\tau_{w}^{L M}$ may be discontinous at $\omega^{*}$ as they switch between $\tau_{w 1}$ and $\tau_{w 2}$ at $\omega^{*}$. This discontinuity is caused by

$M_{g}\left(\omega^{*}\right)=M_{l}\left(\omega^{*}\right)$.

On the other hand, $M_{g}$ and $V_{g}$ are still continuous functions of $\omega$, and so are $M_{l}(\omega)$ and $V_{l}(\omega)$ where they are defined. In addition, $M_{l}\left(\omega^{*}\right)=V_{l}\left(\omega^{*}\right)$ if $M_{l}(\omega)$ and $V_{l}(\omega)$ exist in $\left(\omega^{*}-\varepsilon, \omega^{*}\right]$ and do not exist in $\left(\omega^{*}, \omega^{*}+\varepsilon\right)$, or vice versa.

For the convenience of further development, we introduce the following concept.

Definition 7.1 For a given set $\Omega^{\prime} \subset \mathbb{R}_{+}^{o}$, an interval $\mathcal{I}$ is known as a maximal interval of $\Omega^{\prime}$ if $\mathcal{I} \subset \Omega^{\prime}$, and for any interval $\mathcal{I}^{\prime} \supset \mathcal{I}, \mathcal{I}^{\prime} \neq \mathcal{I}$, there exists an $\omega \in \mathcal{I}^{\prime}$ such that $\omega \notin \Omega^{\prime}$.

We may plot $M_{g}, M_{l}, V_{l}$ and $V_{g}$ against $\omega$ to obtain four curves. Then according to Assumptions V to VII, no two curves may intersect with the $\omega$-axis at the same point, no curve may be tangent to the $\omega$-axis, and no curve may pass through the origin. It can be shown that Assumption III implies that there exists a $\omega_{u b}$ such that

$M_{g}(\omega)<0$ for all $\omega>\omega_{u b}$.
This, together with Assumptions V to VII, implies that each of these four curves intersect with the $\omega$-axis a finite number of times. Using this for $M_{g}(\omega)$ alone, we may arrive at the following proposition.

Proposition 7.2 The crossing frequency set $\Omega$ may be obtained from the function $M_{g}(\omega)$ :

$\Omega=\left\{\omega>0 \mid M_{g}(\omega) \geq 0\right\}$.

The set $\Omega$ may be decomposed into a finite number of its maximal intervals $\Omega_{i}$ of finite length,

$\Omega=\bigcup_{i=1}^{K} \Omega_{i}$

where $\Omega_{i}$ is ordered from the left to the right with increasing i. All $\Omega_{i}$ are closed

$\Omega_{i}=\left[\omega_{i}^{l}, \omega_{i}^{r}\right]$,

with the possible exception of $i=1$ when $M_{g}(0)>0$, in which case

$\Omega_{1}=\left(\omega_{1}^{l}, \omega_{1}^{r}\right]=\left(0, \omega_{1}^{r}\right]$.

An $\omega$ is an end point of some $\Omega_{i}$, i.e., $\omega=\omega_{i}^{l}$ or $\omega=\omega_{i}^{r}$, if and only if

$M_{g}(\omega)=0$,

with the possible exception of $\omega=\omega_{1}^{l}=0$, in which case $M_{g}(\omega)=M_{g}(0)>0$.

Using the remaining functions $M_{l}, V_{l}$ and $V_{g}$ in additional to $M_{g}$, we may define the following subsets of $\Omega$,

$\Omega_{G 1}=\left\{\omega \in \mathbb{R}_{o}^{+} \mid V_{g}(\omega)>0\right\}$,

$\Omega_{G 2 M}=\left\{\omega \in \mathbb{R}_{o}^{+} \mid V_{l}(\omega)<0<M_{l}(\omega)\right\}$,

and

$$
\begin{aligned}
\Omega_{N 1}= & \left\{\omega \in \mathbb{R}_{o}^{+} \mid M_{l}(\omega) \leq 0 \leq M_{g}(\omega)\right\}, \\
\Omega_{N 2}= & \left\{\omega \in \mathbb{R}_{o}^{+} \mid V_{g}(\omega) \leq 0 \leq V_{l}(\omega)\right\}, \\
\Omega_{N 3}= & \left\{\omega \in \mathbb{R}_{o}^{+}\right. \\
& \left.\mid V_{l}(\omega) \text { and } M_{l}(\omega) \text { do not exist, } V_{g}(\omega) \leq 0 \leq M_{g}(\omega)\right\} .
\end{aligned}
$$

Furthermore, we define

$\Omega_{G M}=\Omega_{G 1} \cup \Omega_{G 2 M}$,

$\Omega_{N}=\Omega_{N 1} \cup \Omega_{N 2} \cup \Omega_{N 3}$.

We may state the following proposition. 
Fig. $4 M_{g}(\omega), M_{l}(\omega), V_{l}(\omega)$ and $V_{g}(\omega)$ for the system given in Example 7.4. More detailed views are given in Fig. 5 and Fig. 6

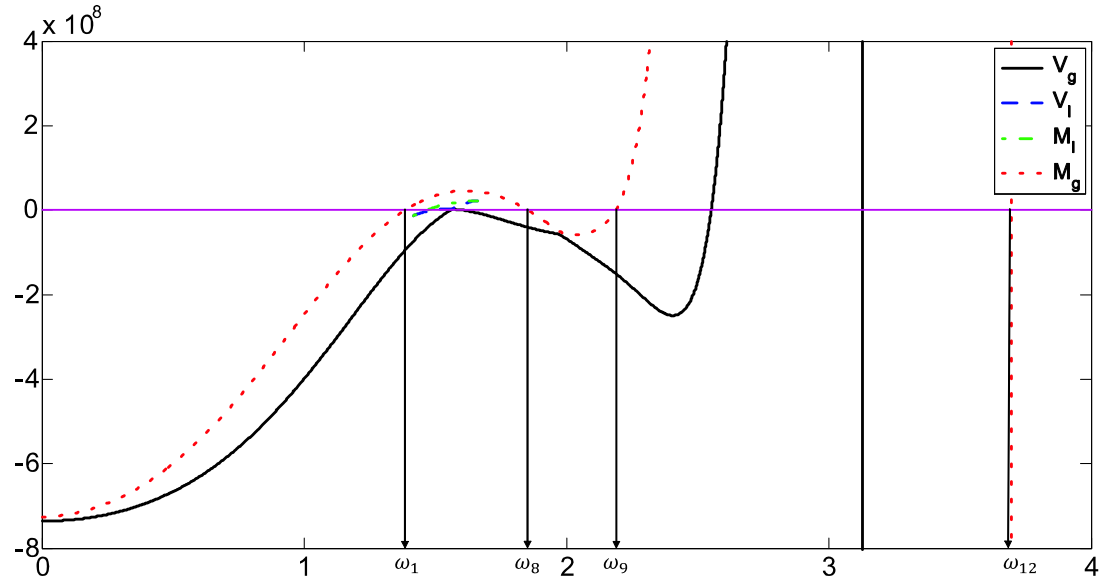

Proposition 7.3 The sets $\Omega_{G 1}, \Omega_{G 2 M}, \Omega_{N 1}, \Omega_{N 2}$ and $\Omega_{N 3}$ are disjoint, and

$\Omega=\Omega_{G M} \cup \Omega_{N}$.

Each maximal interval $\Omega_{i}$ of $\Omega$ may be further partitioned as

$\Omega_{i}=\bigcup_{h=1}^{K_{i}} \Omega_{i}^{h}$,

where each $\Omega_{i}^{h}$ is a maximal interval of either $\Omega_{G M}$ or $\Omega_{N}$, and $\Omega_{i}^{h}$ are ordered from left to right with increasing $h$. If $\Omega_{i}^{h} \subset \Omega_{G M}$, then it is an open interval

$\Omega_{i}^{h}=\left(\omega_{i}^{h l}, \omega_{i}^{h r}\right)$.

If $\Omega_{i}^{h} \subset \Omega_{N}$, then it is a closed interval

$\Omega_{i}^{h}=\left[\omega_{i}^{h l}, \omega_{i}^{h r}\right]$,

with the possible exception of $i=1, h=1$ and $\omega_{1}^{1 l}=0$, in which case it is a semi-open interval

$\Omega_{1}^{1}=\left(\omega_{1}^{1 l}, \omega_{1}^{1 r}\right]=\left(0, \omega_{1}^{1 r}\right]$.

The right most interval must be a maximal interval of $\Omega_{N}$, i.e., $\Omega_{i}^{K_{i}} \subset \Omega_{N}$. The remaing $\Omega_{i}^{h}$ within $\Omega_{i}$ alternates between maximal intervals of $\Omega_{G M}$ and those of $\Omega_{N}$, i.e., $\Omega_{i}^{K_{i}-1} \subset$ $\Omega_{G M}$ (if it exists), $\Omega_{i}^{K_{i}-2} \subset \Omega_{N}$ (if it exists), $\Omega_{i}^{K_{i}-3} \subset \Omega_{G M}$ (if it exists) and so on. If the left most interval $\Omega_{i}^{1}$ is such that $\omega_{i}^{1 l} \neq 0$, then $\Omega_{i}^{1} \subset \Omega_{N}$.

Proof This is obvious from the definitions.

Obviously, the curves $M_{g}(\omega), M_{l}(\omega), V_{l}(\omega)$ and $V_{g}(\omega)$ depend on which $\tau_{w}$ is used with $\omega$ to parameterize $\mathcal{T}$. On the other hand, it will become clear later on that the sets $\Omega_{N}$ and
$\Omega_{G M}$ are independent of the choice of $w$. This also means that the partition of $\Omega$ to $\Omega_{i}$ and $\Omega_{i}^{h}$ are intrinsic property of the system, and is independent of the choice of parameterization.

It should be pointed out that if $\Omega_{i}^{h} \subset \Omega_{G M}$, then $\Omega_{i}^{h}$ must also be a maximal interval of either $\Omega_{G 1}$ or $\Omega_{G 2 M}$. However, if $\Omega_{i}^{h} \subset \Omega_{N}, \Omega_{i}^{h}$ does not have to be a maximal interval of $\Omega_{N 1}$ or $\Omega_{N 2}$ or $\Omega_{N 3}$, as illustrated in Example 7.4 next.

Example 7.4 Consider $\Delta(s)$ in the form of (1.1) with

$$
\begin{aligned}
& p_{0}(s)=\left(-s^{2}+4\right)\left(s^{2}+2 s+0.5\right)\left(s^{2}+s+5\right), \\
& p_{1}(s)=0.5\left(s^{2}+2 s+0.5\right)\left(s^{2}+5 s+6\right) \\
& p_{2}(s)=7\left(s^{2}+4\right)\left(s^{2}+5 s+6\right) \\
& p_{3}(s)=4\left(s^{2}+4\right)\left(s^{2}+2 s+0.5\right) \\
& p_{12}(s)=\left(s^{2}+1\right)(s+0.4)(s+0.7) \\
& p_{23}(s)=(s+0.4)(s+0.7)(s+0.3) \\
& p_{13}(s)=\left(s^{2}+1\right)(s+0.6)(s+0.4) \\
& p_{123}(s)=\left(s^{2}+4\right)(s+1)(s+2)(s+4)
\end{aligned}
$$

The functions $M_{g}(\omega), M_{l}(\omega), V_{l}(\omega)$ and $V_{g}(\omega)$ for this system are plotted in Fig. 4 , with more detailed view given in Fig. 5 and Fig. 6. The approximate numerical values of $\omega_{i}$ are:

$$
\begin{aligned}
& \omega_{1}=1.3873, \omega_{2}=1.4174, \omega_{3}=1.4695 \\
& \omega_{4}=1.4955, \omega_{5}=1.5607, \omega_{6}=1.6017 \\
& \omega_{7}=1.6619, \omega_{8}=1.8537, \omega_{9}=2.1879 \\
& \omega_{10}=2.5516, \omega_{11}=3.1286, \omega_{12}=3.6937
\end{aligned}
$$

In this case,

$\Omega=\Omega_{1} \cup \Omega_{2}=\left[\omega_{1}, \omega_{8}\right] \cup\left[\omega_{9}, \omega_{12}\right]$.

The maximal intervals $\Omega_{1}$ and $\Omega_{2}$ of $\Omega$ are further partitioned into maximal intervals $\Omega_{i}^{h}$ of either $\Omega_{N}$ or $\Omega_{G M}$. Specifically, 
Fig. 5 Detailed view of Fig. 4 for $\omega$ in the range of $[1.3,2.6]$

Fig. 6 Detailed view of Fig. 4 for $\omega$ in the range of $[3,3.8]$
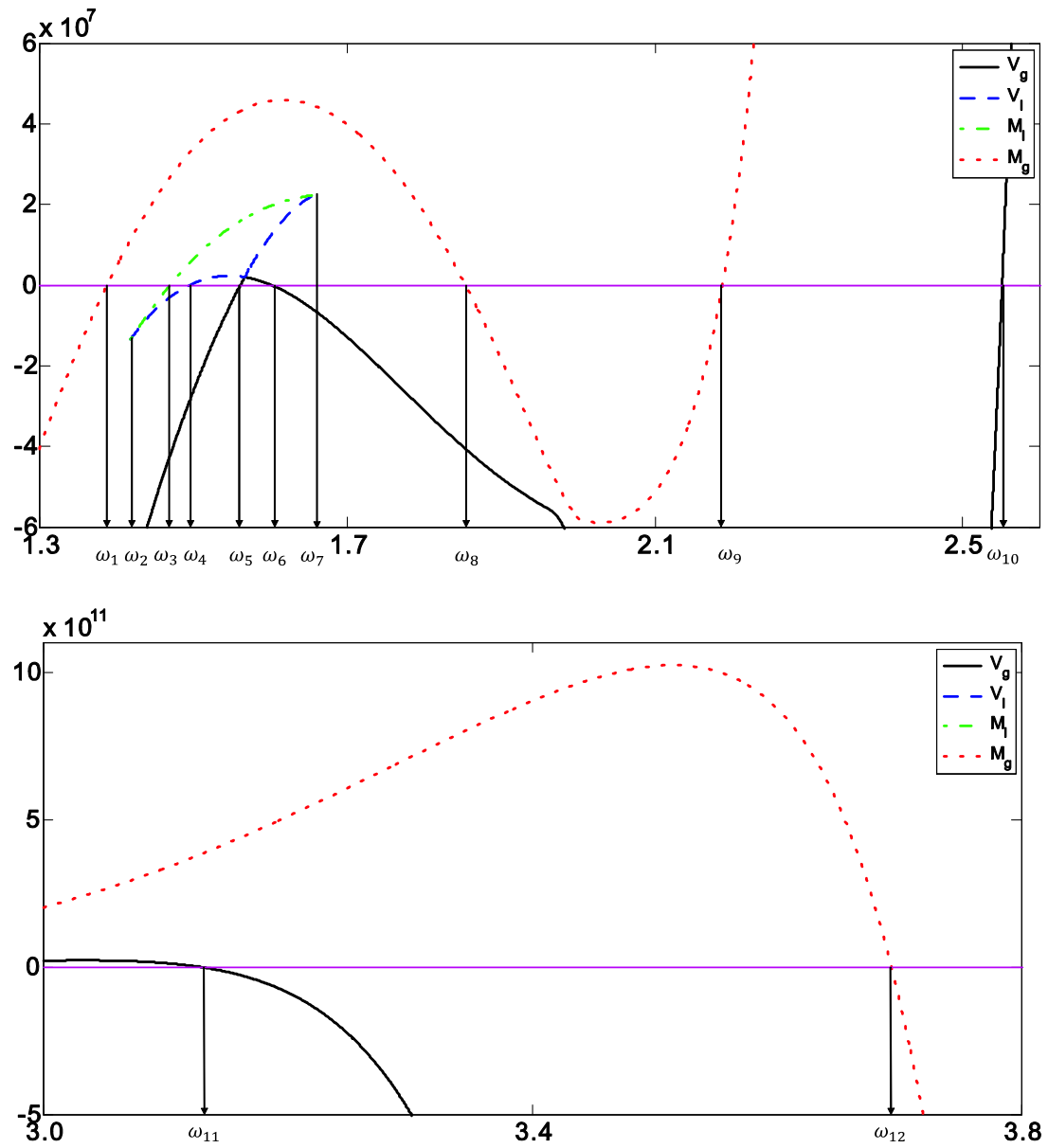

$\Omega_{1}=\bigcup_{h=1}^{5} \Omega_{1}^{h}$,

$\Omega_{2}=\bigcup_{h=1}^{3} \Omega_{2}^{h}$,

where

$\Omega_{1}^{1}=\left[\omega_{1}, \omega_{3}\right] \subset \Omega_{N}, \Omega_{1}^{2}=\left(\omega_{3}, \omega_{4}\right) \subset \Omega_{G 2 M}$,

$\Omega_{1}^{3}=\left[\omega_{4}, \omega_{5}\right] \subset \Omega_{N}, \Omega_{1}^{4}=\left(\omega_{5}, \omega_{6}\right) \subset \Omega_{G 1}$,

$\Omega_{1}^{5}=\left[\omega_{6}, \omega_{8}\right] \subset \Omega_{N}$;

$\Omega_{2}^{1}=\left[\omega_{9}, \omega_{10}\right] \subset \Omega_{N}, \Omega_{2}^{2}=\left(\omega_{10}, \omega_{11}\right) \subset \Omega_{G 1}$,

$\Omega_{2}^{3}=\left[\omega_{11}, \omega_{12}\right] \subset \Omega_{N}$.

It should be noted that $\Omega_{1}^{1}$ and $\Omega_{1}^{5}$ are not maximal intervals of $\Omega_{N 1}$ or $\Omega_{N 2}$ or $\Omega_{N 3}$. Indeed, $\Omega_{1}^{1}=\left[\omega_{1}, \omega_{2}\right) \cup\left[\omega_{2}, \omega_{3}\right]$, where $\left[\omega_{1}, \omega_{2}\right) \subset \Omega_{N 3},\left[\omega_{2}, \omega_{3}\right] \subset \Omega_{N 1}$. Similarly, $\Omega_{1}^{5}=$ $\left[\omega_{6}, \omega_{7}\right] \cup\left(\omega_{7}, \omega_{8}\right]$ where $\left[\omega_{6}, \omega_{7}\right] \subset \Omega_{N 2},\left(\omega_{7}, \omega_{8}\right] \subset \Omega_{N 3}$.

If $\Omega_{i}^{h} \subset \Omega_{G 1}$, then for any given $\omega \in \Omega_{i}^{h}, \phi\left(\omega, \tau_{w}\right)$ as a function of $\tau_{w}$ can be illustrated as in Fig. 7. In this case, $\left(\omega, \tau_{w}\right) \in \Lambda^{(w) o}$ for all $\tau_{w} \in \mathbb{R}$, and $M_{l}(\omega)$ and $V_{l}(\omega)$ may or may not exist for any given $\omega \in \Omega_{i}^{h}$.

If $\Omega_{i}^{h} \subset \Omega_{G 2 M}$, then for any given $\omega \in \Omega_{i}^{h}, \phi\left(\omega, \tau_{w}\right)$ as a function of $\tau_{w}$ can be illustrated as in Fig. 8. In this

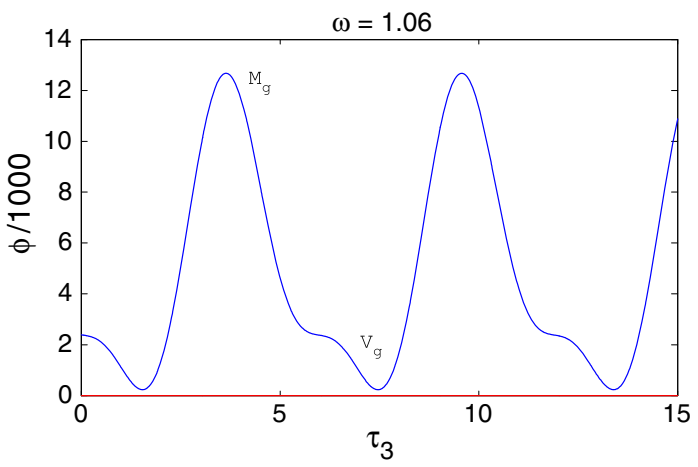

Fig. 7 A typical $\phi\left(\omega, \tau_{w}\right)$ vs. $\tau_{w}$ for $\omega \in \Omega_{G 1}$. This figure is generated from the system given in Example 5.3 with $\omega=1.06$

case, an appropriate $2 \pi / \omega$ range contains two maximal $\tau_{w}$ -intervals. Let these two intervals be denoted as $\left[\tau_{w}^{a}, \tau_{w}^{b}\right]$ and $\left[\tau_{w}^{c}, \tau_{w}^{d}\right]$. Then, $\tau_{w}^{a}, \tau_{w}^{b}, \tau_{w}^{c}$ and $\tau_{w}^{d}$ may be chosen as continuous functions of $\omega$ within $\Omega_{i}^{h}$. Furthermore, the types of $\left(\omega, \tau_{w}^{a}\right),\left(\omega, \tau_{w}^{b}\right),\left(\omega, \tau_{w}^{c}\right)$ and $\left(\omega, \tau_{w}^{d}\right)$ remain unchanged as $\omega$ varies within $\Omega_{i}^{h}$ in view of Corallary 5.2.

If $\Omega_{i}^{h} \subset \Omega_{N}$, then for any given $\omega \in \Omega_{i}^{h o}=\left(\omega_{i}^{h l}, \omega_{i}^{h r}\right)$ (i.e., $\omega$ in the interior of $\left.\Omega_{i}^{h}\right), \phi\left(\omega, \tau_{w}\right)$ as a function of $\tau_{w}$ may be either as shown in Fig. 9 (when $\omega \in \Omega_{N 1}$ ), or as shown 


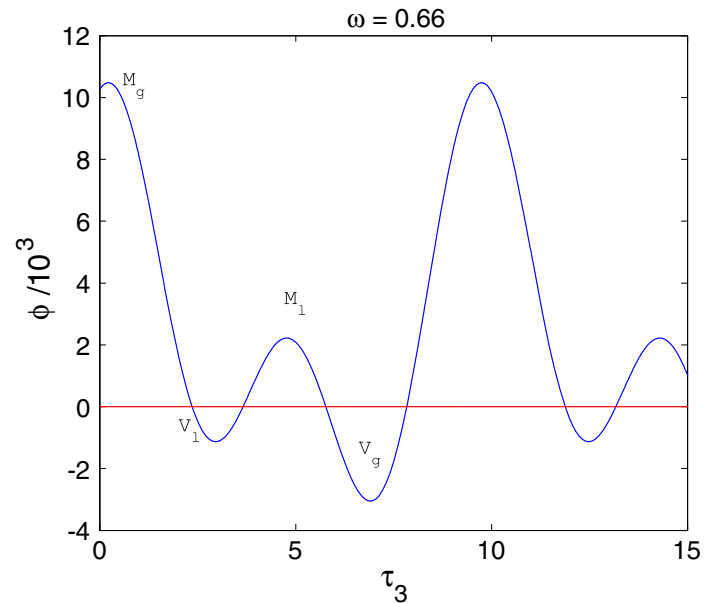

Fig. 8 A typical $\phi\left(\omega, \tau_{w}\right)$ vs. $\tau_{w}$ for $\omega \in \Omega_{G 2 M}$. This figure is generated from the system given in Example 5.3 with $\omega=0.66$

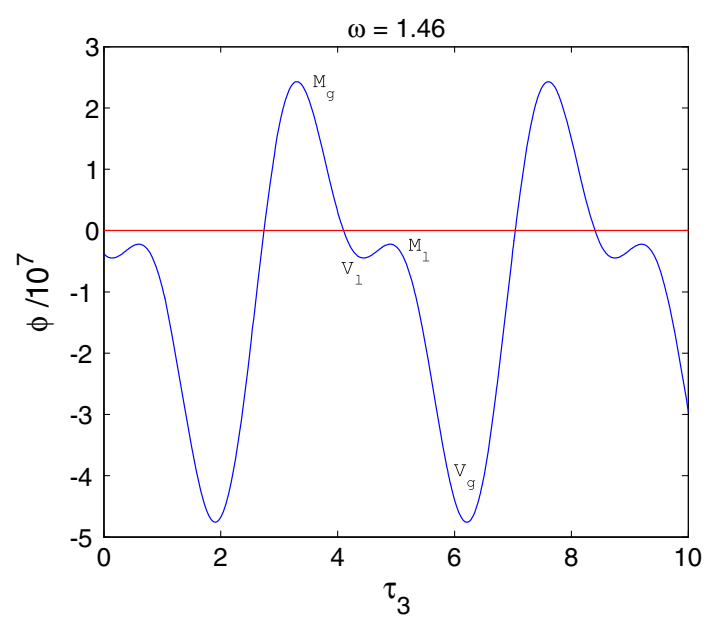

Fig. 9 A typical $\phi\left(\omega, \tau_{w}\right)$ vs. $\tau_{w}$ for $\omega \in \Omega_{N 1}$. This figure is generated from the system given in Example 7.4. with $\omega=1.46$

in Fig. 10 (when $\omega \in \Omega_{N 2}$ ), or as shown in Fig. 11 (when $\omega \in \Omega_{N 3}$ ). In this case, an appropriate $2 \pi / \omega$ range contains one maximal $\tau_{w}$-interval. Let this interval be $\left[\tau_{a}, \tau_{b}\right]$. Then $\tau_{a}$ and $\tau_{b}$ may be chosen as continuous functions of $\omega$ within $\Omega_{i}^{h o}$, and the types of $\left(\omega, \tau_{w}^{a}\right)$ and $\left(\omega, \tau_{w}^{b}\right)$ remain unchanged as $\omega$ varies within $\Omega_{i}^{\text {ho }}$ in view of Corallary 5.2.

It should be pointed out that if $\omega \in \Omega_{N}$, and $\left[\tau_{w}^{a}, \tau_{w}^{b}\right]$ is a maximal $\tau_{w}$-interval, then any maximal $\tau_{w}$-interval can be expressed as $\left[\tau_{w}^{a}+2 \pi k / \omega, \tau_{w}^{b}+2 \pi k / \omega\right]$ for an appropriate integer $k$. Similarly, for an $\omega \in \Omega_{G 2 M}$, let $\left[\tau_{w}^{a}, \tau_{w}^{b}\right]$ and $\left[\tau_{w}^{c}, \tau_{w}^{d}\right]$ be two maximal $\tau_{w}$-intervals within a $2 \pi / \omega$ range, then any maximal $\tau_{w}$-interval can be expressed as either $\left[\tau_{w}^{a}+2 \pi k / \omega, \tau_{w}^{b}+2 \pi k / \omega\right]$ or $\left[\tau_{w}^{c}+2 \pi k / \omega, \tau_{w}^{d}+2 \pi k / \omega\right]$. Furthermore, the type invariance in a continuous piece of boundary (Corollary 5.2) imposes some constraints between the types of the end points of these maximal $\tau_{w}$-intervals as specified in thefollowing proposition.

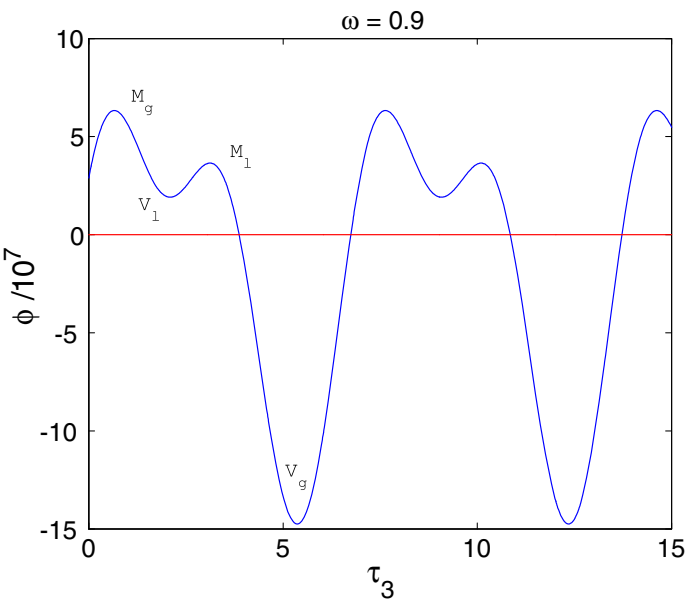

Fig. 10 A typical $\phi\left(\omega, \tau_{w}\right)$ vs. $\tau_{w}$ for $\omega \in \Omega_{N 2}$. This figure is generated from the system given in Example 5.3 with $\omega=0.9$

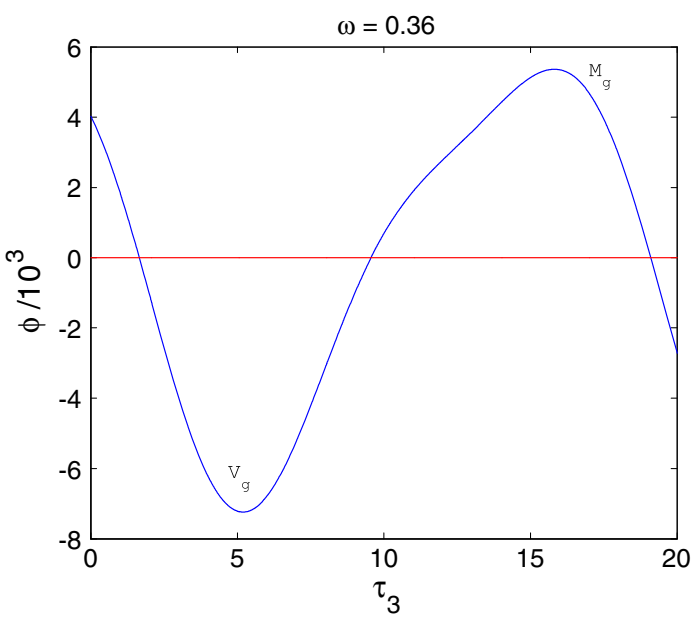

Fig. 11 A typical $\phi\left(\omega, \tau_{w}\right)$ vs. $\tau_{w}$ for $\omega \in \Omega_{N 3}$. This figure is generated from the system given in Example 5.3 with $\omega=0.36$

Proposition 7.5 If $\omega \in \Omega_{i}^{h o} \subset \Omega_{N}$, and $\left[\tau_{w}^{a}, \tau_{w}^{b}\right]$ is a maximal $\tau_{w}$-interval, then

$\operatorname{Type}\left(\omega, \tau_{w}^{a}\right)=\operatorname{Type}\left(\omega, \tau_{w}^{b}\right)$.

If $\omega \in \Omega_{G 2 M}$, and $\left[\tau_{w}^{a}, \tau_{w}^{b}\right]$ and $\left[\tau_{w}^{c}, \tau_{w}^{d}\right]$ are two maximal $\tau_{w}$-intervals such that

$\tau_{w}^{a}<\tau_{w}^{b}<\tau_{w}^{c}<\tau_{w}^{d}<\tau_{w}^{a}+2 \pi / \omega$,

then either

$\operatorname{Type}\left(\omega, \tau_{w}^{a}\right)=\operatorname{Type}\left(\omega, \tau_{w}^{d}\right)$,

$\operatorname{Type}\left(\omega, \tau_{w}^{b}\right)=\operatorname{Type}\left(\omega, \tau_{w}^{c}\right)$,

or

$\operatorname{Type}\left(\omega, \tau_{w}^{a}\right)=\operatorname{Type}\left(\omega, \tau_{w}^{b}\right)$,

$\operatorname{Type}\left(\omega, \tau_{w}^{c}\right)=\operatorname{Type}\left(\omega, \tau_{w}^{d}\right)$. 
Proof For $\omega \in \Omega_{i}^{h o}$, if $\Omega_{i}^{h} \subset \Omega_{N}$, let Type $\left(\omega, \tau_{w}^{a}\right)=a$, Type $\left(\omega, \tau_{w}^{b}\right)=b$. If $\Omega_{i}^{h} \subset \Omega_{G 2 M}$, let also Type $\left(\omega, \tau_{w}^{c}\right)=c$, Type $\left(\omega, \tau_{w}^{d}\right)=d$. The invariance of boundary type means that $a, b, c$ and $d$ do not change within $\Omega_{i}^{h o}$. Similarly, if $h \neq K_{i}$ let $\omega^{+} \in \Omega_{i}^{h+1}$. Then if $\Omega_{i}^{h+1} \subset \Omega_{N}$, let $\left[\tau_{w}^{a+}, \tau_{w}^{b+}\right]$ be a maximal $\tau_{w}$-interval, and Type $\left(\omega^{+}, \tau_{w}^{a+}\right)=$ $a^{+}$, Type $\left(\omega^{+}, \tau_{w}^{b+}\right)=b^{+}$, and if $\Omega_{i}^{h+1} \subset \Omega_{G 2 M}$ let $\left[\tau_{w}^{c+}, \tau_{w}^{d+}\right]$ be another maximal $\tau_{w}$-interval such that

$\tau_{w}^{a+}<\tau_{w}^{b+}<\tau_{w}^{c+}<\tau_{w}^{d+}<\tau_{w}^{a+}+2 \pi / \omega$

and Type $\left(\omega^{+}, \tau_{w}^{c+}\right)=c^{+}$, Type $\left(\omega^{+}, \tau_{w}^{d+}\right)=d^{+}$. We will use induction to prove the proposition as $h$ decreases from $K_{i}$ : first show that the conclusion is true for $h=K_{i}$, then show that it is true for $h$ under the assumption that it is true for $h+1$. These two conclusions imply that the conclusion is true for all $1 \leq h \leq K_{i}$.

For $h=K_{i}$, then $\Omega_{i}^{h} \subset \Omega_{N}$ (Proposition 7.3), and $M_{g}\left(\omega_{i}^{h r}\right)=0$. In this case, $\tau_{w}^{b}-\tau_{w}^{a} \downarrow 0$ as $\omega \uparrow \omega_{i}^{h r}$. This implies $a=b$ according to type invariance (Corollary 5.2). Therefore, the proposition is true for $h=K_{i}$. It remains to be shown that the conclusion is true for $\Omega_{i}^{h}$ under the assumption that it is true for $\Omega_{i}^{h+1}$. There are three cases to prove.

i) $\Omega_{i}^{h+1} \subset \Omega_{N}$. In this case, the inductive assumption is $a^{+}=b^{+}$, and Proposition 7.3 requires either $\Omega_{i}^{h} \subset \Omega_{G 1}$, or $\Omega_{i}^{h} \subset \Omega_{G 2 M}$.

a) $\Omega_{i}^{h} \subset \Omega_{G 1}$. Then there is nothing to prove, and the proposition is vacuously true.

b) $\Omega_{i}^{h} \subset \Omega_{G 2 M}$. Then, either $M_{l}\left(\omega_{i}^{h r}\right)=0$ or $V_{l}\left(\omega_{i}^{h r}\right)=0$.

1) If $M_{l}\left(\omega_{i}^{h r}\right)=0$, then as $\omega \uparrow \omega_{i}^{h r}$, one of the two intervals $\left[\tau_{w}^{a}, \tau_{w}^{b}\right]$ and $\left[\tau_{w}^{c}, \tau_{w}^{d}\right]$, say $\left[\tau_{w}^{c}, \tau_{w}^{d}\right]$, is reduced to one point, and the other, say $\left[\tau_{w}^{a}, \tau_{w}^{b}\right]$, continues to become $\left[\tau_{w}^{a+}+2 \pi k / \omega, \tau_{w}^{b+}+\right.$ $2 \pi k / \omega]$ for some integer $k$. We may thus conclude (7.9) and (7.10) from type invariance (Corollary 5.2) and the inductive assumption.

2) If $V_{l}\left(\omega_{i}^{h r}\right)=0$, then as $\omega \uparrow \omega_{i}^{h r}=\omega_{i}^{h+1, l}$, either $\left[\tau_{w}^{a}, \tau_{w}^{b}\right]$ and $\left[\tau_{w}^{c}, \tau_{w}^{d}\right]$ merge into one interval $\left[\tau_{w}^{a+}+2 \pi k / \omega, \tau_{w}^{b+}+2 \pi k / \omega\right]$ for some $k$, or $\left[\tau_{w}^{c}, \tau_{w}^{d}\right]$ and $\left[\tau_{w}^{a}+2 \pi / \omega, \tau_{w}^{b}+2 \pi / \omega\right]$ merge into one interval. In the first case,

$$
\begin{aligned}
\lim _{\omega \uparrow \omega_{i}^{h r}} \tau_{w}^{b} & =\lim _{\omega \uparrow \omega_{i}^{h r}} \tau_{w}^{c}, \\
\lim _{\omega \uparrow \omega_{i}^{h r}} \tau_{w}^{a} & =\lim _{\omega \downarrow \omega_{i}^{h+1, l}}\left(\tau_{w}^{a+}+2 \pi k / \omega\right), \\
\lim _{\omega \uparrow \omega_{i}^{h r}} \tau_{w}^{d} & =\lim _{\omega \downarrow \omega_{i}^{h+1, l}}\left(\tau_{w}^{b+}+2 \pi k / \omega\right),
\end{aligned}
$$

which imply (Corollary 5.2)

$b=c$,

$a=a^{+}$,

$d=b^{+}$.

The above and the inductive assumption $a^{+}=$ $b^{+}$imply (7.7) and (7.8). In the second case,

$$
\begin{aligned}
& \lim _{\omega \uparrow \omega_{i}^{h r}} \tau_{w}^{d}=\lim _{\omega \uparrow \omega_{i}^{h r}}\left(\tau_{w}^{a}+2 \pi / \omega\right), \\
& \lim _{\omega \uparrow \omega_{i}^{h r}} \tau_{w}^{c}=\lim _{\omega \downarrow \omega_{i}^{h+1, l}}\left(\tau_{w}^{a+}+2 \pi k / \omega\right), \\
& \lim _{\omega \uparrow \omega_{i}^{h r}}\left(\tau_{w}^{b}+2 \pi / \omega\right)=\lim _{\omega \downarrow \omega_{i}^{h+1, l}}\left(\tau_{w}^{b+}+2 \pi k / \omega\right) .
\end{aligned}
$$

Similar to the first case, we may conclude (7.7) and (7.8) from inductive assumption and the type invariance.

ii) $\Omega_{i}^{h+1} \subset \Omega_{G 1}$. In this case, Proposition 7.3 requires $\Omega_{i}^{h} \subset \Omega_{N}$, and $V_{g}\left(\omega_{l}^{h r}\right)=0$. As $\omega \uparrow \omega_{i}^{h r}$, all maximal $\tau_{w}$-intervals merge together. Especially, $\left[\tau_{w}^{a}, \tau_{w}^{b}\right]$ merges with $\left[\tau_{w}^{a}+2 \pi / \omega, \tau_{w}^{b}+2 \pi / \omega\right]$, i.e., $\tau_{w}^{b}-\tau_{w}^{a} \uparrow 2 \pi / \omega$. As the functions defining boundary types on the left hand side of (5.5) to (5.8) are all periodic functions, we may conclude (7.5).

iii) $\Omega_{i}^{h+1} \subset \Omega_{G 2 M}$. In this case, Proposition 7.3 requires $\Omega_{i}^{h} \subset \Omega_{N}$. Then, either $M_{l}\left(\omega_{i}^{h r}\right)=0$ or $V_{l}\left(\omega_{i}^{h r}\right)=0$. The inductive assumptions are either

$a^{+}=d^{+}$,
$b^{+}=c^{+}$,

or

$a^{+}=b^{+}$,

$c^{+}=d^{+}$.

We need to show

$a=b$.

a) $M_{l}\left(\omega_{i}^{h r}\right)=0$. Then as $\omega \downarrow \omega_{i}^{h r}$, one of the two maximal $\tau_{w}$-intervals, say $\left[\tau_{w}^{a+}, \tau_{w}^{b+}\right]$, reduces to one point, the other, say $\left[\tau_{w}^{c+}, \tau_{w}^{d+}\right]$, continues to become $\left[\tau_{w}^{a}+2 \pi k / \omega, \tau_{w}^{b}+2 \pi k / \omega\right]$ for some integer $k$. This means

$a^{+}=b^{+}$,

which, together with the inductive assumptions, imply $c^{+}=d^{+}$, which in turn means (7.16) in view of type invariance (Corollary 5.2). 
b) $V_{l}\left(\omega_{i}^{h r}\right)=0$. Then as $\omega \downarrow \omega_{i}^{h r}$, two maximal $\tau_{w^{-}}$ intervals $\left[\tau_{w}^{a+}, \tau_{w}^{b+}\right]$ and $\left[\tau_{w}^{c+}, \tau_{w}^{d+}\right]$ or $\left[\tau_{w}^{c+}, \tau_{w}^{d+}\right]$ and $\left[\tau_{w}^{a+}+2 \pi / \omega, \tau_{w}^{b+}+2 \pi / \omega\right]$ merge into one interval $\left[\tau_{w}^{a}+2 \pi k / \omega, \tau_{w}^{b}+2 \pi k / \omega\right]$. In the first case,

$c^{+}=b^{+}$,

which together with the inductive assumptions, implies $a^{+}=d^{+}$. As

$$
\begin{aligned}
\lim _{\omega \downarrow \omega_{i}^{h+1, l}} \tau_{w}^{a+} & =\lim _{\omega \uparrow \omega_{i}^{h r}}\left(\tau_{w}^{a}+2 \pi k / \omega\right), \\
\lim _{\omega \downarrow \omega_{i}^{h+1, l}} \tau_{w}^{d+} & =\lim _{\omega \uparrow \omega_{i}^{h r}}\left(\tau_{w}^{b}+2 \pi k / \omega\right),
\end{aligned}
$$

we conclude (7.16) by type invariance (Corollary 5.2). For the second case,

$a^{+}=d^{+}$,

which together with the inductive assumptions implies $c^{+}=b^{+}$, from which we can again conclude $a=b$ from type invariance (Corollary 5.2).

All the possibilities have now been exhausted, and the proof is complete.

\section{Maximal interval classification}

In this section, we will classify all $\Omega_{i}^{h}$. It turns out that some critical geometric characteristics of the corresponding stability crossing set is completely determined by the type of the interval, as will be shown in the next section.

First, we need to divide $\Omega_{G 2 M}$ into two separate sets. Recall that for a given $\omega \in \Omega_{G 2 M}$, we may find two maximal $\tau_{w}$-intervals $\left[\tau_{w}^{a}, \tau_{w}^{b}\right]$ and $\left[\tau_{w}^{c}, \tau_{w}^{d}\right]$ such that

$\tau_{w}^{a}<\tau_{w}^{b}<\tau_{w}^{c}<\tau_{w}^{d}<\tau_{w}^{a}+2 \pi / \omega$.

The division of $\Omega_{G 2 M}$ is according to the types of the end points of the maximal $\tau_{w}$-intervals,

$\Omega_{G 2}=\left\{\omega \in \Omega_{G 2 M} \mid \operatorname{Type}\left(\omega, \tau_{w}^{a}\right) \neq \operatorname{Type}\left(\omega, \tau_{w}^{b}\right)\right\}$,

$\Omega_{M}=\left\{\omega \in \Omega_{G 2 M} \mid \operatorname{Type}\left(\omega, \tau_{w}^{a}\right)=\operatorname{Type}\left(\omega, \tau_{w}^{b}\right)\right\}$.

Define also

$\Omega_{G}=\Omega_{G 1} \cup \Omega_{G 2}$.

Then

$\Omega=\Omega_{G} \cup \Omega_{N} \cup \Omega_{M}$
It is not difficult to see that any maximal interval $\Omega_{i}^{h}$ defined in Proposition 7.2 is also a maximal interval of either $\Omega_{G}$, or $\Omega_{N}$, or $\Omega_{M}$. We will call $\Omega_{G}$ the Grashof set, $\Omega_{N}$ the non-Grashof set of the first kind, and $\Omega_{M}$ the non-Grashof set of the second kind. These terms are adapted from the case without the cross terms discussed in [22] although they no longer have any direct correspondence with the four-bar linkage theory discussed in [29].

From the discussions in Sect. 6, for an $\omega \in \Omega_{G}$, the corresponding constant frequency curves are spirals. For a given

$\boldsymbol{\tau}=\delta_{1} \boldsymbol{\tau}_{1}^{0}+\delta_{2} \tau_{2}^{0}+\delta_{3} \tau_{3}^{0}$,

where

$\delta_{i} \in\{-1,0,1\}, i=1,2,3$, and at least one $\delta_{i} \neq 0$,

we define $\Omega_{G}^{\tau}$ as those $\omega \in \Omega_{G}$ that has constant frequency curves with axis $\tau$. From the discussions in Sect. 6, we have $\delta_{3}=1$ for all $\omega \in \Omega_{G 1}$, and $\delta_{3}=0$ for all $\omega \in \Omega_{G 2}$. Obviously

$\Omega_{G}=\bigcup_{\tau} \Omega_{G}^{\tau}$,

where the union includes all $\boldsymbol{\tau}$ with the expression (8.1) with $\delta_{i}, i=1,2,3$ going through the set described by (8.2). We also consider $\Omega_{G}^{-\tau}$ the same set as $\Omega_{G}^{\tau}$ as $-\tau$ and $\tau$ represent the same axis.

Proposition 8.1 A maximal interval $\Omega_{i}^{h}$ of $\Omega_{G 1}$ or $\Omega_{G 2}$ is also a maximal interval of $\Omega_{G}^{\tau}$ for some $\boldsymbol{\tau}$.

Proof For a maximal interval $\Omega_{i}^{h}$ of $\Omega_{G 1}$ or $\Omega_{G 2}$, it is sufficient to show that $\boldsymbol{\tau}$ is independent of $\omega$ within $\Omega_{i}^{h}$.

Consider first the case of $\Omega_{i}^{h} \subset \Omega_{G 1}$. Recall for each $\omega \in \Omega_{i}^{h}$,

$\boldsymbol{\tau}=\delta_{t_{w}}\left(P_{u}^{(w)}(\omega, \cdot)\right) \boldsymbol{\tau}_{u}^{0}+\delta_{t_{w}}\left(P_{v}^{(w)}(\omega, \cdot)\right) \boldsymbol{\tau}_{v}^{0}+\boldsymbol{\tau}_{w}^{0}$.

From Proposition 6.6, as $\omega$ changes continuously, the rotation index $\delta_{t_{w}}\left(P_{u}^{(w)}(\omega, \cdot)\right)$ or $\delta_{t_{w}}\left(P_{v}^{(w)}(\omega, \cdot)\right)$ may change its value only at those values of $\omega$ such that

$P_{u}^{(w)}\left(\omega, \tau_{w}\right)=0$, or

$P_{v}^{(w)}\left(\omega, \tau_{w}\right)=0$

for some $\tau_{w}$. However, this is not possible for $\omega \in \Omega_{i}^{h}$ in view of Proposition 4.3 because $\left(\omega, \tau_{w}\right) \in \Lambda^{(w)}$ for all $\omega \in \Omega_{i}^{h}$ and $\tau_{w} \in \mathbb{R}$. Therefore, $\boldsymbol{\tau}$ is indeed identical for all $\omega \in \Omega_{i}^{h}$.

We now consider the other case, $\Omega_{i}^{h} \subset \Omega_{G 2}$. Since the types of $\left(\omega, \tau_{a}\right)$ and $\left(\omega, \tau_{b}\right)$ do not change with $\omega$ within $\Omega_{i}^{h}$, according to discussion of Case ii) after Proposition 6.4, the axes of spirals (which are the constant frequency curves) do not change with $\omega$, either. 
For an $\omega \in \Omega_{i}^{h o} \subset \Omega_{N}$, let $\left[\tau_{w}^{a}, \tau_{w}^{b}\right]$ be a maximal $\tau_{w^{-}}$ interval. Recall

$\operatorname{Type}\left(\omega, \tau_{w}^{a}\right)=\operatorname{Type}\left(\omega, \tau_{w}^{b}\right)$.

For a given $k \in\{1,2,3,4\}$, we define $\Omega_{N}^{k o}$ as the set of such $\omega$ with Type $\left(\omega, \tau_{w}^{a}\right)=k$, and

$\Omega_{N}^{k}=\overline{\Omega_{N}^{k o}}$

Obviously,

$\Omega_{N}=\bigcup_{k=1}^{4} \Omega_{N}^{k}$,

and a maximal interval of $\Omega_{N}$ is also a maximal interval of $\Omega_{N}^{k}$ for some $k$. The process of defining $\Omega_{N}^{k o}$ first then taking the closure is necessary because some $\omega=\omega_{i}^{h l}$ or $\omega_{i}^{h r}$ may satisfy $V_{l}(\omega)=0$ or $M_{l}(\omega)=0$, and there are two maximal $\tau_{w}$-intervals within a $2 \pi / \omega$ range in such cases.

For an $\omega \in \Omega_{M}$, let $\left[\tau_{w}^{a}, \tau_{w}^{b}\right]$ and $\left[\tau_{w}^{c}, \tau_{w}^{d}\right]$ be two maximal $\tau_{w}$-intervals within a $2 \pi / \omega$ range. For given $k, l \in$ $\{1,2,3,4\}$, we define

$\Omega_{M}^{k l}=\left\{\omega \in \Omega_{M} \mid \operatorname{Type}\left(\omega, \tau_{w}^{a}\right)=k, \operatorname{Type}\left(\omega, \tau_{w}^{c}\right)=l\right\}$.

According to the definition of $\Omega_{M}$, we also have

$\operatorname{Type}\left(\omega, \tau_{w}^{b}\right)=k$,

$\operatorname{Type}\left(\omega, \tau_{w}^{d}\right)=l$.

As $\left[\tau_{w}^{c}, \tau_{w}^{d}\right]$ and $\left[\tau_{w}^{a}+2 \pi / \omega, \tau_{w}^{b}+2 \pi / \omega\right]$ are also within a $2 \pi / \omega$ range,

$\Omega_{M}^{k l}=\Omega_{M}^{l k}$

Obviously,

$\Omega_{M}=\bigcup_{k=1}^{4} \bigcup_{l=1}^{k} \Omega_{M}^{k l}$

and a maximal interval of $\Omega_{M}$ is also a maximal interval of $\Omega_{M}^{k l}$ for some $k$ and $l$.

Again, any maximal interval $\Omega_{i}^{h}$ of $\Omega_{G M}$ or $\Omega_{N}$ is also a maximal interval of $\Omega_{G}^{\tau}$ for some $\tau$, or $\Omega_{N}^{k}$ for some $k$, or $\Omega_{M}^{k l}$ for some $k$ and $l$. Next, we will classify these $\Omega_{i}^{h}$ according to which subsets $\Omega_{i}^{h}$ and its neighbors belong. The subset that $\Omega_{i}^{h}$ belongs to is represented by the letter $G, N$ or $M$ with appropriate supercript. The two subscripts represent the subsets that $\Omega_{i}^{h-1}$ and $\Omega_{i}^{h+1}$ belong to, or if $\Omega_{i}^{h}$ is the left most or the right most subinterval in $\Omega_{i}$. Specifically:
For an $\Omega_{i}^{h} \subset \Omega_{G}^{\tau}$, if $\Omega_{i}^{h-1} \subset \Omega_{N}^{k}, \Omega_{i}^{h+1} \subset \Omega_{N}^{l}$, then we say $\Omega_{i}^{h}$ is of type $G_{k l}^{\tau}$. If $\omega_{i}^{h l}=0$ instead, then its type is $G_{<l}^{\boldsymbol{\tau}}$.

For an $\Omega_{i}^{h} \subset \Omega_{M}^{m n}$, if $\Omega_{i}^{h-1} \subset \Omega_{N}^{k}, \Omega_{i}^{h+1} \subset \Omega_{N}^{l}$, then $\Omega_{i}^{h}$ is said to be of type $M_{k l}^{m n}$. If $\omega_{i}^{h l}=0$ instead, then its type is $M_{<l}^{m n}$.

For an $\Omega_{i}^{h} \subset \Omega_{N}^{k}$, if $\Omega_{i}^{h-1} \subset \Omega_{G}, \Omega_{i}^{h+1} \subset \Omega_{G}$, then we say $\Omega_{i}^{h}$ is of type $N_{G G}^{k}$. If $\Omega_{i}^{h+1} \subset \Omega_{M}$ instead, then $\Omega_{i}^{h}$ is of type $N_{G M}^{k}$. We may define type $N_{M G}^{k}$ and $N_{M M}^{k}$ analogously. If $\omega_{i}^{h l}=0$ and $\Omega_{i}^{h+1} \subset \Omega_{G}$, then $\Omega_{i}^{h}$ is of type $N_{<G}^{k}$. If $\omega_{i}^{h l}=\omega_{i}^{l} \neq 0$ instead, then $\Omega_{i}^{h}$ is of type $N_{* G}^{k}$. If $\Omega_{i}^{h-1, o} \subset \Omega_{G}$ and $\omega_{i}^{h r}=\omega_{i}^{r}$, then $\Omega_{i}^{h}$ is of type $N_{G *}^{k}$. The types $N_{<M}^{k}, N_{<*}^{k}, N_{* M}^{k}, N_{* *}^{k}$, and $N_{M *}^{k}$ are defined analogously.

The above list is exhaustive according to Proposition 7.3.

\section{Geometric Characterization}

Define

$\mathcal{T}_{i}^{h o}=\bigcup_{\omega \in \Omega_{i}^{h o}} \mathcal{T}_{\omega}$,

$\mathcal{T}_{i}^{h}=\overline{\mathcal{T}_{i}^{h o}}$

Then

$\mathcal{T}=\bigcup_{i=1}^{K} \bigcup_{h=1}^{K_{i}} \mathcal{T}_{i}^{h} \bigcap \mathbb{R}_{+}^{3}=\bigcup_{i=1}^{K} \mathcal{T}_{i} \bigcap \mathbb{R}_{+}^{3}$,

where

$\mathcal{T}_{i}=\bigcup_{h=1}^{K_{i}} \mathcal{T}_{i}^{h}$

The key to understanding the geometric characteristics of the stability crossing set $\mathcal{T}$ lies in the understanding of $\mathcal{T}_{i}^{h}$ and how it is connected with the remaining part of $\mathcal{T}_{i}$. It turns out that the most important geometric characteristics of $\mathcal{T}_{i}^{h}$ are completely determined by the type of $\Omega_{i}^{h}$ as stated in the next theorem.

Theorem 9.1 If $\Omega_{i}^{h}$ is of type $G_{k k}^{\tau}$, then $\mathcal{T}_{i}^{h}$ consists of an array of pipes with axis $\tau$ and variable cross sections, each of them also contains two series of holes. If $\Omega_{i}^{h}$ is of type $G_{k l}^{\tau}, k \neq l$, then $\mathcal{T}_{i}^{h}$ is a series of wavy sheets, each of them contains an array of holes. If $\Omega_{i}^{h}$ is of type $G_{<k}^{\tau}$, then $\mathcal{T}_{i}^{h}$ consists of an array of open surfaces, each of them extends to infinity and contains a series of holes. If $\Omega_{i}^{h}$ is of type $N_{G G}^{k}, N_{G M}^{k}, N_{M G}^{k}$, or $N_{M M}^{k}$, then $\mathcal{T}_{i}^{h}$ consists of a lattice of connectors, each of them connects a hole in $\mathcal{T}_{i}^{h-1}$ and a hole in $\mathcal{T}_{i}^{h+1}$. If $\Omega_{i}^{h}$ is of type $N_{* G}^{k}, N_{G *}^{k}, N_{* M}^{k}$ or $N_{M *}^{k}$, then $\mathcal{T}_{i}^{h}$ 
consists of a lattice of caps, each fits a hole in either $\mathcal{T}_{i}^{h-1}$ or $\mathcal{T}_{i}^{h+1}$. If $\Omega_{i}^{h}$ is of type $N_{* *}^{k}$, then $\mathcal{T}_{i}^{h}$ consists of a lattice of closed surfaces. If $\Omega_{i}^{h}$ is of type $N_{<*}^{k}$, then $\mathcal{T}_{i}^{h}$ consists of a lattice of open caps that extend to infinity. If $\Omega_{i}^{h}$ is of type $N_{<G}^{k}$ or $N_{<M}^{k}$, then $\mathcal{T}_{i}^{h}$ consists of a lattice of semi-open pipes, each fits a hole of $\mathcal{T}_{i}^{h+1}$ at one end, and extends to infinity at the other end. If $\Omega_{i}^{h}$ is of type $M_{k k}^{m n}$ with $m \neq n$, then $\mathcal{T}_{i}^{h}$ consists of a lattice of closed surfaces and a lattice of connectors, each connector connectes a hole in $\mathcal{T}_{i}^{h-1}$ and a hole in $\mathcal{T}_{i}^{h+1}$. If $\Omega_{i}^{h}$ is of type $M_{k l}^{m n}$ with $m \neq n, k \neq l$, then $\mathcal{T}_{i}^{h}$ consists of two lattices of caps, one lattice of caps fit the holes of $\mathcal{T}_{i}^{h-1}$, the others fit the holes of $\mathcal{T}_{i}^{h+1}$. If $\Omega_{i}^{h}$ is of type $M_{<l}^{m n}, m \neq n$, then $\mathcal{T}_{i}^{h}$ consists of a lattice of open caps and a lattice of semi-open pipes; each open cap extends to infinity, and each semi-open pipe contains a hole at one end that fits a hole in $\mathcal{T}_{i}^{h+1}$ and the other end extends to infinity.

The above theorem may be summarized in the following table

\begin{tabular}{|c|c|c|c|c|}
\hline$\Omega_{i}^{h}$ & $\Omega_{i}^{h-1}$ & $\Omega_{i}^{h+1}$ & Type & $\mathcal{T}_{i}^{h}$ \\
\hline \multirow[t]{2}{*}{$\Omega_{G}^{\tau}$} & $\Omega_{N}^{k}$ & $\Omega_{N}^{l}$ & $G_{k l}^{\tau}$ & $\begin{array}{l}\text { Pipes if } k=l \\
\text { Wavy sheets if } \\
k \neq l\end{array}$ \\
\hline & 0 & $\Omega_{N}^{k}$ & $G_{<k}^{\boldsymbol{\tau}}$ & Open surfaces \\
\hline \multirow[t]{6}{*}{$\Omega_{N}^{k}$} & $\Omega_{G M}$ & $\Omega_{G M}$ & $\begin{array}{l}N_{G G}^{k}, N_{G M}^{k}, \\
\quad N_{M G}^{k} \text {, or } N_{M M}^{k}\end{array}$ & Connectors \\
\hline & $\omega_{i}^{l}$ & $\Omega_{G M}$ & $N_{* G}^{k}$ or $N_{* M}^{k}$ & Caps \\
\hline & $\Omega_{G M}$ & $\omega_{i}^{r}$ & $N_{G *}^{\hat{k}}$ or $N_{M *}^{\hat{k}}$ & Caps \\
\hline & $\omega_{i}^{l}$ & $\omega_{i}^{r}$ & $N_{* *}^{k}$ & Closed surfaces \\
\hline & 0 & $\omega_{i}^{r}$ & $N_{<*}^{k}$ & Open caps \\
\hline & 0 & $\Omega_{G M}$ & $N_{<G}^{k}$ or $N_{<M}^{k}$ & Semi-open pipes \\
\hline \multirow[t]{2}{*}{$\Omega_{M}^{m n} m \neq n$} & $\Omega_{N}^{k}$ & $\Omega_{N}^{l}$ & $M_{k l}^{m n}$ & $\begin{array}{l}\text { Closed surfaces } \\
\& \text { connectors } \\
\text { if } k=l \\
\text { Two caps if } k \neq l\end{array}$ \\
\hline & 0 & $\Omega_{N}^{l}$ & $M_{<l}^{m n}$ & $\begin{array}{l}\text { Open caps \& } \\
\text { semi-open } \\
\text { pipes }\end{array}$ \\
\hline
\end{tabular}

Note that the case $\Omega_{i}^{h} \subset \Omega_{M}^{k k}$ has not been addressed in the above theorem. This case is rather special as the geometry of such a case may not be completely determined by its neighbors. We will discuss this case in the appendix.

In the remaining part of this section, we will provide specific details of all the cases given in Theorem 9.1. These details also serve as a proof of the theorem.

Corresponding to each maximal interval $\Omega_{i}^{h}$ of various types covered in Theorem 9.1, the stability crossing set $\mathcal{T}_{i}^{h}$ consists of different pieces of surfaces that can be easily parameterized by $\left(\omega, \tau_{w}\right)$ based on the constant frequency curves discussed in Sect. 6. A close observation of these surfaces indicates that some of them are connected to form a larger surface of certain geometric shapes mentioned in Theorem 9.1. While these are derived using parameters $\left(\omega, \tau_{w}\right)$, the geometric characteristics are instrinsic in nature, and are independent of parameterization. Indeed, in some cases, it is more convenient to describe these surfaces using parameters $\left(\omega, \tau_{u}\right)$ or $\left(\omega, \tau_{v}\right)$ rather than $\left(\omega, \tau_{w}\right)$, similar to the description of constant frequency curves in Case ii) in Proposition 6.4. In the following, we will carry out these descriptions for all the cases covered in Theorem 9.1. The description is divided in three cases: Case i) is for $\Omega_{i}^{h} \subset \Omega_{N}^{k}$, Case ii) is for $\Omega_{i}^{h} \subset \Omega_{M}^{m n}$, and Case iii) is for $\Omega_{i}^{h} \subset \Omega_{G}^{\tau}$. Each case also contains several subcases.

Case i) $\Omega_{i}^{h} \subset \Omega_{N}^{k}$,

From (6.1) and (6.2) in Sect. 6, a curve corresponding to an $\omega \in \Omega_{i}^{h o}$ and a maximal $\tau_{w}$-iinterval $\left[\tau_{w}^{a}, \tau_{w}^{b}\right]$ is

$$
\begin{aligned}
\mathcal{T}_{\omega,\left[\tau_{w}^{a}, \tau_{w}^{b}\right]}^{(w) k_{u} k_{v} \pm} & =\bigcup_{\tau_{w}^{a} \leq \tau_{w} \leq \tau_{w}^{b}}\left\{\left(\tau_{1}, \tau_{2}, \tau_{3}\right) \mid \tau_{u}\right. \\
& \left.=\tau_{u}^{k_{u} \pm}\left(\omega, \tau_{w}\right), \tau_{v}=\tau_{v}^{k_{v} \pm}\left(\omega, \tau_{w}\right)\right\}
\end{aligned}
$$

for a given $k_{u}, k_{v}$ and + or - . In the above, $\tau_{w}^{a}$ and $\tau_{w}^{b}$ also depends on $\omega$. Recognizing $\left[\tau_{w}^{a}+2 k_{w} \pi / \omega, \tau_{w}^{b}+2 k_{w} \pi / \omega\right]$ is also a maximal $\tau_{w}$-interval, we define

$\mathcal{T}_{\omega,\left[\tau_{w}^{a}, \tau_{w}^{b}\right]}^{(w) k_{u} k_{v} k_{w} \pm}=\mathcal{T}_{\omega,\left[\tau_{w}^{a}+2 k_{w} \pi / \omega, \tau_{w}^{b}+2 k_{w} \pi / \omega\right]}^{(w) k_{k} k_{v} \pm}$

From

$\tau_{u}^{k_{u} \pm}\left(\omega, \tau_{w}+2 k_{w} \pi / \omega\right)=\tau_{u}^{k_{u} \pm}\left(\omega, \tau_{w}\right)$,

$\tau_{v}^{k_{v} \pm}\left(\omega, \tau_{w}+2 k_{w} \pi / \omega\right)=\tau_{v}^{k_{v} \pm}\left(\omega, \tau_{w}\right)$,

we see that $\mathcal{T}_{\omega,\left[\tau_{w}^{a}, \tau_{w}^{b}\right]}^{(w) k_{k} k_{v} k_{w} \pm}$ can be obtained from $\mathcal{T}_{\omega,\left[\tau_{w}^{a}, \tau_{w}^{b}\right]}^{(w) k_{k} k_{v} \pm}$ by moving $2 k_{w} \pi / \omega$ along the $\boldsymbol{\tau}_{w}^{0}$-direction. We can define a patch of surface

$\mathcal{T}_{i}^{h(w) k_{u} k_{v} k_{w} \pm}=\overline{\bigcup_{\omega \in \Omega_{i}^{h o}} \mathcal{T}_{\omega,\left[\tau_{w}^{a}, \tau_{w}^{b}\right]}^{(w) k_{u} k_{v} k_{w} \pm}}$

where we agree that $\tau_{w}^{a}$ and $\tau_{w}^{b}$ are chosen such that they are continuous functions of $\omega$ within $\Omega_{i}^{h}$. Due to the difference of $\omega$ within $\Omega_{i}^{h}, \mathcal{T}_{i}^{h(w) k_{u} k_{v} k_{w} \pm}$ for different $k_{u}, k_{v}$ and $k_{w}$ have similar shapes but are not identical. Furthermore, $\mathcal{T}_{i}^{h(w) k_{u} k_{v} k_{w}+}$ and $\mathcal{T}_{i}^{h(w) k_{u}^{\prime} k_{v}^{\prime} k_{w}-}$ may be connected to form a larger surface for some $k_{u}^{\prime}$ and $k_{v}^{\prime}$ depending on the value of $k$ in a way very similar to the constant frequency curves discussed in Case i) after Proposition 6.4. We will call this larger surface $\mathcal{T}_{i}^{h(w) k_{u} k_{v} k_{w}}$. Specifically,

$$
\begin{aligned}
& \mathcal{T}_{i}^{h(w) k_{u} k_{v} k_{w}} \\
& =\mathcal{T}_{i}^{h(w) k_{u} k_{v} k_{w}+} \bigcup \mathcal{T}_{i}^{h(w) k_{u}, k_{v}-1, k_{w}-} \text { for } \Omega_{i}^{h} \subset \Omega_{N}^{1}, \\
& \mathcal{T}_{i}^{h(w) k_{u} k_{v} k_{w}} \\
& =\mathcal{T}_{i}^{h(w) k_{u} k_{v} k_{w}+} \bigcup \mathcal{T}_{i}^{h(w) k_{u}+1, k_{v}, k_{w}-} \text { for } \Omega_{i}^{h} \subset \Omega_{N}^{2},
\end{aligned}
$$


Fig. 12 Connector

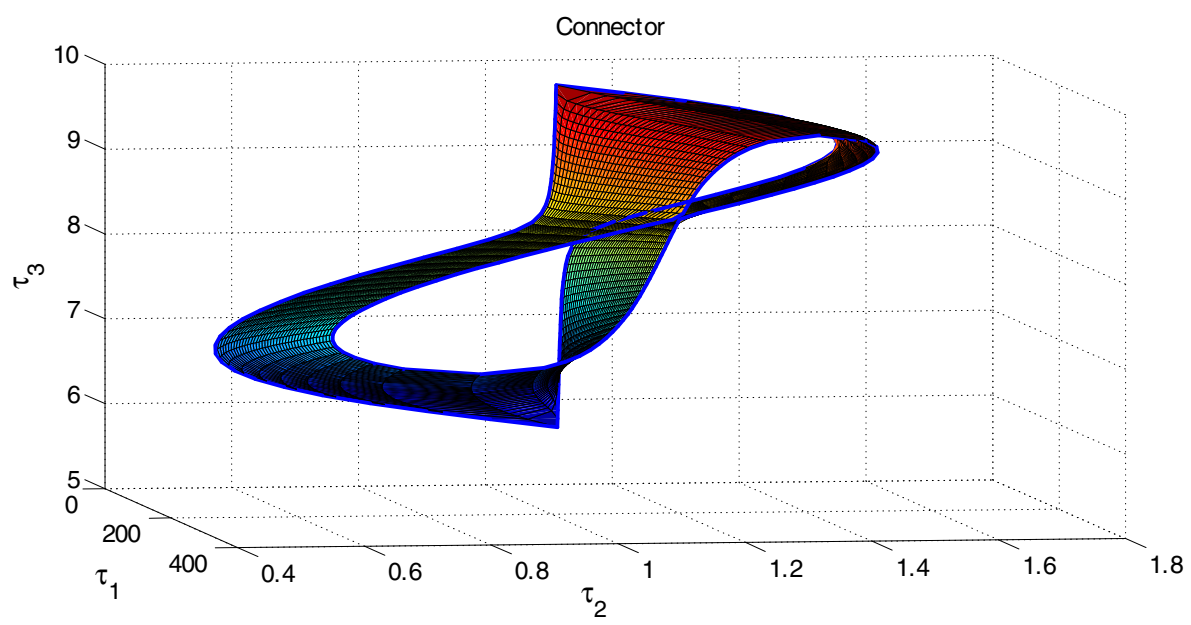

$$
\begin{aligned}
& \mathcal{T}_{i}^{h(w) k_{u} k_{v} k_{w}} \\
& =\mathcal{T}_{i}^{h(w) k_{u} k_{v} k_{w}+} \bigcup \mathcal{T}_{i}^{h(w) k_{u}+1, k_{v}-1, k_{w}-} \text { for } \Omega_{i}^{h} \subset \Omega_{N}^{3}, \\
& \mathcal{T}_{i}^{h(w) k_{u} k_{v} k_{w}} \\
& =\mathcal{T}_{i}^{h(w) k_{u} k_{v} k_{w}+} \bigcup \mathcal{T}_{i}^{h(w) k_{u} k_{v}, k_{w}-} \text { for } \Omega_{i}^{h} \subset \Omega_{N}^{4} .
\end{aligned}
$$

This surface can also be defined from the constant frequency curves

$$
\mathcal{T}_{i}^{h(w) k_{u} k_{v} k_{w}}=\overline{\bigcup_{\omega \in \Omega_{i}^{h o}} \mathcal{T}_{\omega,\left[\tau_{w}^{a}+2 k_{w} \pi / \omega, \tau_{w}^{b}+2 k_{w} \pi / \omega\right]}^{(a)}},
$$

where $\mathcal{T}_{\omega,\left[\tau_{w}^{a}, \tau_{w}^{b}\right]}^{(w) k_{u} k_{v}}$ is a closed curve defined in (6.3), (6.5), (6.6) or (6.7) depending on $k$. Obviously,

$\mathcal{T}_{i}^{h}=\bigcup_{k_{w}=-\infty}^{+\infty} \bigcup_{k_{v}=-\infty}^{+\infty} \bigcup_{k_{u}=-\infty}^{+\infty} \mathcal{T}_{i}^{h(w) k_{u} k_{v} k_{w}}$

In other words, $\mathcal{T}_{i}^{h}$ consists of a lattice of surfaces $\mathcal{T}_{i}^{h(w) k_{u} k_{v} k_{w}}$. We can therefore conclude the following from the situation at the two ends of $\Omega_{i}^{h}$.

Case i.a) $\Omega_{i}^{h-1} \subset \Omega_{G M}, \Omega_{i}^{h+1} \subset \Omega_{G M}$.

In this case, $\mathcal{T}_{\omega,\left[\tau_{w}^{a}, \tau_{w}^{b}\right]}^{(w) k_{u} k_{v} k_{w}}$ remains as a closed curve as $\omega \uparrow$ $\omega_{i}^{h r}$ or $\downarrow \omega_{i}^{h l}$. Therefore, $\mathcal{T}_{i}^{h(w) k_{u} k_{v} k_{w}}$ is a connector with two ends fitting holes in $\mathcal{T}_{i}^{h-1}$ and $\mathcal{T}_{i}^{h+1}$.

Example 9.2 Consider $\Omega_{1}^{3}=\left[\omega_{4}, \omega_{5}\right]$ in the system given in Example 7.4. For $u=1, v=2$ and $w=3$, it can be verified that $\Omega_{1}^{3}$ is of type $N_{M G}^{1}$. Therefore, the corresponding $\mathcal{T}_{1}^{3}$ consists of a lattice of connectors. One of these connectors is shown in Fig. 12. An interesting observation is that the closed curves for any $\omega \in\left(\omega_{4}, \omega_{5}\right]$ are all simple curves, but the curve at $\omega_{4}$ evolve into two closed curves. This is natural if we consider the left neighbor of $\Omega_{1}^{3}$. Indeed $\Omega_{1}^{2} \in \Omega_{M}^{11}$ with $M_{l}\left(\omega_{1}^{2 l}\right)=0, V_{l}\left(\omega_{1}^{2 r}\right)=0$. As will be shown in the next section, the corresponding $\mathcal{T}_{1}^{2}$ consists of an array of connectors and an array of caps. A connector and a cap fit the two closed curves at $\omega_{4}$.

Case i.b) $\omega_{i}^{h l}=\omega_{i}^{l}, \Omega_{i}^{h+1} \subset \Omega_{G M}$

In this case, $\mathcal{T}_{\omega,\left[\tau_{w}^{a}, \tau_{w}^{b}\right]}^{(w) k_{u} k_{w} k_{w}}$ remains as a closed curve as $\omega \uparrow$ $\omega_{i}^{h r}$. On the other hand, from

$M_{g}\left(\omega_{i}^{h l}\right)=\phi\left(\omega_{i}^{h l}, \tau_{w}^{G M}\right)=0$

we conclude that as $\omega \downarrow \omega_{i}^{h l}, \tau_{w}^{a} \rightarrow \tau_{w}^{G M}+2 \pi k^{\prime} / \omega$ and $\tau_{w}^{b} \rightarrow \tau_{w}^{G M}+2 \pi k^{\prime} / \omega$ for some integer $k^{\prime}$, and the curve $\mathcal{T}_{\omega,\left[\tau_{w}^{a}, \tau_{w}^{b}\right]}^{(w) k_{u} k_{v} k_{w}}$ is reduced to one point, i.e. the surface $\mathcal{T}_{i}^{h(w) k_{u} k_{v} k_{w}}$ is closed on this end. Therefore, $\mathcal{T}_{i}^{h(w) k_{u} k_{v} k_{w}}$ is a cap, with one end closed and the other end fits a hole of $\mathcal{T}_{i}^{h+1}$.

Example 9.3 Consider $\Omega_{2}^{1}=\left[\omega_{9}, \omega_{10}\right]$ in the system given in Example 7.4. For $u=1, v=2$ and $w=3$, it can be verified that $\Omega_{2}^{1}$ is of type $N_{* G}^{3}$. Therefore, the corresponding $\mathcal{T}_{2}^{1}$ consists of a lattice of caps. One of such caps is shown in Fig. 13

Case i.c) $\Omega_{i}^{h-1} \subset \Omega_{G M}, \omega_{i}^{h r}=\omega_{i}^{r}$

This is very similar to case i.b), $\mathcal{T}_{i}^{h(w) k_{u} k_{v} k_{w}}$ is again a cap, except the cap fits a hole of $\mathcal{T}_{i}^{h-1}$.

Case i.d) $\omega_{i}^{h l}=\omega_{i}^{l}, \omega_{i}^{h r}=\omega_{i}^{r}$

In this case, $\mathcal{T}_{\omega,\left[\tau_{w}^{a}, \tau_{w}^{b}\right]}^{(w) k_{u} k_{v} k_{w}}$ is reduced to a point as $\omega \uparrow \omega_{i}^{h r}$ or $\downarrow \omega_{i}^{h l}$. This means that $\mathcal{T}_{i}^{h(w) k_{u} k_{v} k_{w}}$ is a closed surface.

Case i.e) $\omega_{i}^{h l}=0, \Omega_{i}^{h+1} \subset \Omega_{G M}$

In this case, $\mathcal{T}_{\omega,\left[\tau_{w}^{a}, \tau_{w}^{b}\right]}^{(w) k_{u} k_{v} k_{w}}$ remains as a closed curve as $\omega \uparrow$ $\omega_{i}^{h r}$. However, as $\omega \downarrow \omega_{i}^{h l}=0, \mathcal{T}_{\omega,\left[\tau_{w}^{a}, \tau_{w}^{b}\right]}^{(w) k_{u} k_{v} k_{w}} \rightarrow \infty$. Therefore, $\mathcal{T}_{i}^{h(w) k_{u} k_{v} k_{w}}$ is a semi-open pipe, with one end fits a hole of $\mathcal{T}_{i}^{h+1}$, the other end is "open", i.e., approaches $\infty$. 
Fig. 13 A cap

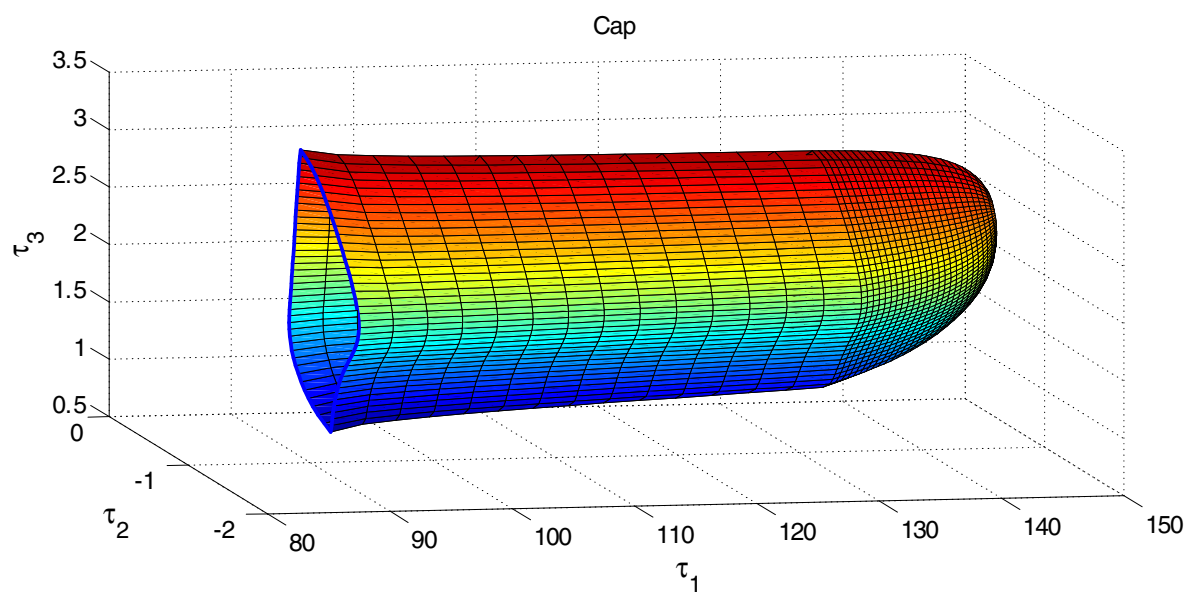

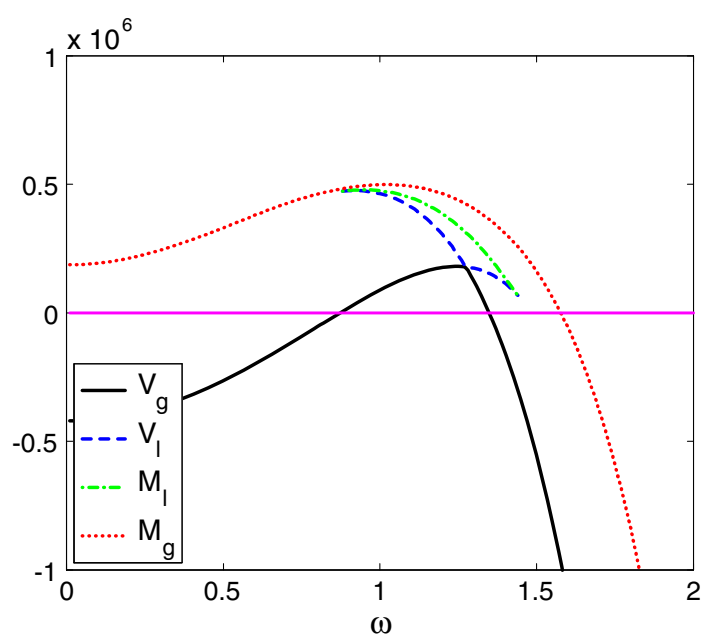

Fig. $14 M_{g}(\omega), M_{l}(\omega), V_{l}(\omega)$, and $V_{g}(\omega)$ for the system given in Example 9.4

Example 9.4 Consider the system (1.1) with

$$
\begin{aligned}
& p_{0}(s)=s^{3}+10 s^{2}+31 s+16, \\
& p_{1}(s)=s^{2}+11 s+28, \\
& p_{2}(s)=s^{2}+5 s-10, p_{3}(s)=1, \\
& p_{12}(s)=2 s+1, p_{23}(s)=3 s+4, \\
& p_{31}(s)=s+5, p_{123}(s)=1 .
\end{aligned}
$$

With For $u=1, v=2$ and $w=3$, Fig. 14 can be plotted, and it can be seen that $\Omega_{1}^{1}=(0,0.8723]$. It can be verified that $\Omega_{1}^{1}$ is of type $N_{<G}^{2}$. Therefore, the corresponding $\mathcal{T}_{1}^{1}$ consists of a lattice of semi-open pipes, one of which is shown in Fig. 15.

$$
\text { Case i.f) } \omega_{i}^{h l}=0, \omega_{i}^{h r}=\omega_{i}^{r}
$$

In this case, $\mathcal{T}_{\omega,\left[\tau_{w}^{a}, \tau_{w}^{b}\right]}^{(w) k_{u} k_{w} k_{w}}$ reduces to one point as $\omega \uparrow \omega_{i}^{h r}$, and it approaches $\infty$ as $\omega \downarrow \omega_{i}^{h l}=0$. Therefore, $\mathcal{T}_{i}^{h(w) k_{u} k_{v} k_{w}}$ is an open cap, which is closed on one end, and approaches $\infty$ at the other end.
Example 9.5 Consider the system (1.1) with

$$
\begin{aligned}
& p_{0}(s)=s^{3}+10 s^{2}+31 s+30, \\
& p_{1}(s)=s^{2}+11 s+28, \\
& p_{2}(s)=s^{2}+5 s+7, p_{3}(s)=1, \\
& p_{12}(s)=2 s+1, p_{23}(s)=3 s+4, \\
& p_{31}(s)=s+5, p_{123}(s)=1 .
\end{aligned}
$$

For $u=1, v=2$ and $w=3$, Fig. 16 can be plotted, and $\Omega=\Omega_{1}^{1}=\left(0, \omega_{1}^{1 r}\right]$, where $\omega_{1}^{1 r}=\omega_{1}^{r} \approx 1.8606$. It can be seen that $\Omega_{1}^{1}$ is of type $N_{<*}^{3}$, and the corresponding $\mathcal{T}_{i}^{h}$ consists of a lattice of open caps, one of which is shown in Fig. 17.

Case ii) $\Omega_{i}^{h} \subset \Omega_{M}^{m n}, m \neq n$

In this case, for each $\omega \in \Omega_{i}^{h}$, there are two $\tau_{w}$-maximal intervals $\left[\tau_{w}^{a}, \tau_{w}^{b}\right]$ and $\left[\tau_{w}^{c}, \tau_{w}^{d}\right], \tau_{w}^{a}<\tau_{w}^{b}<\tau_{w}^{c}<\tau_{w}^{d}<$ $\tau_{w}^{a}+2 \pi / \omega$, and

Type $\left(\omega, \tau_{w}^{a}\right)=\operatorname{Type}\left(\omega, \tau_{w}^{b}\right)=m$,

Type $\left(\omega, \tau_{w}^{c}\right)=\operatorname{Type}\left(\omega, \tau_{w}^{d}\right)=n$.

Similar to (9.1), (9.2) and (9.3), we may define

$$
\begin{aligned}
& \mathcal{T}_{\omega,\left[\tau_{w}^{a}, \tau_{w}^{b}\right]}^{(w) k_{u} k_{v} \pm}=\bigcup_{\tau_{w}^{a} \leq \tau_{w} \leq \tau_{w}^{b}}\left\{\left(\tau_{1}, \tau_{2}, \tau_{3}\right) \mid \tau_{u}\right. \\
& \left.=\tau_{u}^{k_{u} \pm}\left(\omega, \tau_{w}\right), \tau_{v}=\tau_{v}^{k_{v} \pm}\left(\omega, \tau_{w}\right)\right\}, \\
& \mathcal{T}_{\omega,\left[\tau_{w}^{c}, \tau_{w}^{d}\right]}^{(w) k_{u} k_{v} \pm}=\bigcup_{\tau_{w}^{c} \leq \tau_{w} \leq \tau_{w}^{d}}\left\{\left(\tau_{1}, \tau_{2}, \tau_{3}\right) \mid \tau_{u}=\tau_{u}^{k_{u} \pm}\left(\omega, \tau_{w}\right), \tau_{v}\right. \\
& \left.=\tau_{v}^{k_{v} \pm}\left(\omega, \tau_{w}\right)\right\}, \\
& \mathcal{T}_{\omega,\left[\tau_{w}^{a}, \tau_{w}^{b}\right]}^{(w) k_{w} k_{v} k_{w} \pm}=\mathcal{T}_{\omega,\left[\tau_{w}^{a}+2 k_{w} \pi / \omega, \tau_{w}^{b}+2 k_{w} \pi / \omega\right]}^{\left.(w) k_{v} k_{v}\right]}, \\
& \mathcal{T}_{\omega,\left[\tau_{w}^{c}, \tau_{w}^{d}\right]}^{(w) k_{k} k_{v} k_{w} \pm}=\mathcal{T}_{\omega,\left[\tau_{w}^{c}+2 k_{w} \pi / \omega, \tau_{w}^{d}+2 k_{w} \pi / \omega\right]}^{(w) k_{v} \pm},
\end{aligned}
$$


Fig. 15 A semi-open pipe for the system given in Example 9.4

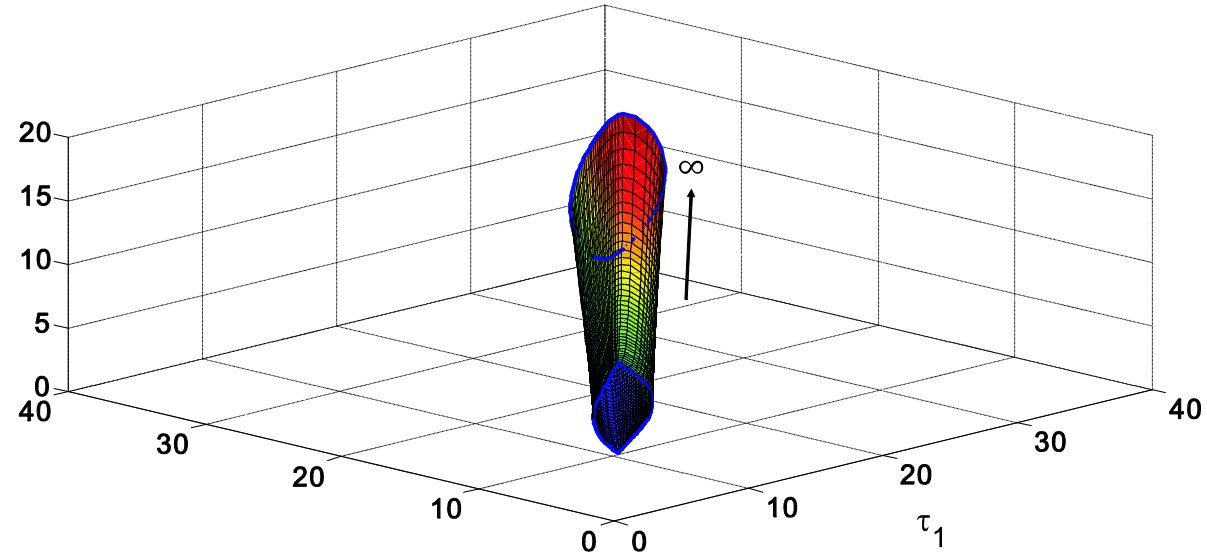

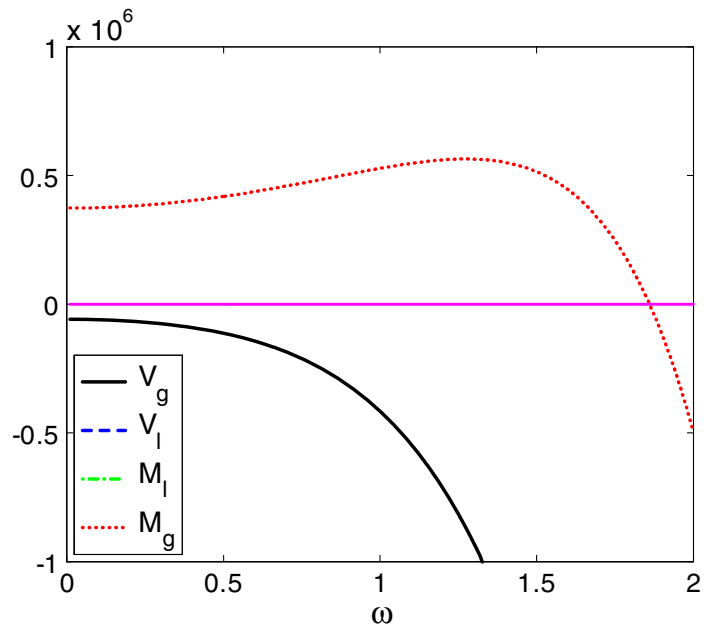

Fig. $16 M_{g}(\omega), M_{l}(\omega), V_{l}(\omega)$, and $V_{g}(\omega)$ for the system given in Example 9.5. In the range shown, $M_{l}(\omega)$ and $V_{l}(\omega)$ do not exist

and

$$
\begin{aligned}
& \mathcal{T}_{i a b}^{h(w) k_{u} k_{v} k_{w} \pm}=\overline{\bigcup_{\omega \in \Omega_{i}^{h}} \mathcal{T}_{\omega,\left[\tau_{w}^{u}, \tau_{w}^{b}\right]}^{(w) k_{k} k_{v} k_{w} \pm}}, \\
& \mathcal{T}_{i c d}^{h(w) k_{u} k_{v} k_{w} \pm}=\overline{\bigcup_{\omega \in \Omega_{i}^{h}} \mathcal{T}_{\omega,\left[\tau_{w}^{c}, \tau_{w}^{d}\right]}^{(w) k_{u} k_{v} k_{w} \pm}} .
\end{aligned}
$$

Larger patches are formed from two patches depending on the types $m$ and $n$,

$$
\begin{aligned}
& \mathcal{T}_{i a b}^{h(w) k_{u} k_{v} k_{w}}=\mathcal{T}_{i a b}^{h(w) k_{u} k_{v} k_{w}+} \bigcup \mathcal{T}_{i a b}^{h(w) k_{u}, k_{v}-1, k_{w}-} \text { for } m=1, \\
& \mathcal{T}_{i a b}^{h(w) k_{u} k_{v} k_{w}}=\mathcal{T}_{i a b}^{h(w) k_{u} k_{v} k_{w}+} \bigcup \mathcal{T}_{i a b}^{h(w) k_{u}+1, k_{v}, k_{w}-} \text { for } m=2, \\
& \mathcal{T}_{i a b}^{h(w) k_{u} k_{v} k_{w}}=\mathcal{T}_{i a b}^{h(w) k_{u} k_{v} k_{w}+} \bigcup \mathcal{T}_{i a b}^{h(w) k_{u}+1, k_{v}-1, k_{w}-} \text { for } m=3, \\
& \mathcal{T}_{i a b}^{h(w) k_{u} k_{v} k_{w}}=\mathcal{T}_{i a b}^{h(w) k_{u} k_{v} k_{w}+} \bigcup \mathcal{T}_{i a b}^{h(w) k_{u} k_{v}, k_{w}-} \text { for } m=4 ;
\end{aligned}
$$

and

$$
\begin{aligned}
& \mathcal{T}_{i c d}^{h(w) k_{u} k_{v} k_{w}}=\mathcal{T}_{i c d}^{h(w) k_{u} k_{v} k_{w}+} \bigcup \mathcal{T}_{i c d}^{h(w) k_{u}, k_{v}-1, k_{w}-} \text { for } n=1, \\
& \mathcal{T}_{i c d}^{h(w) k_{u} k_{v} k_{w}}=\mathcal{T}_{i c d}^{h(w) k_{u} k_{v} k_{w}+} \bigcup \mathcal{T}_{i c d}^{h(w) k_{u}+1, k_{v}, k_{w}-} \text { for } n=2, \\
& \mathcal{T}_{i c d}^{h(w) k_{u} k_{v} k_{w}}=\mathcal{T}_{i c d}^{h(w) k_{u} k_{v} k_{w}+} \bigcup \mathcal{T}_{i c d}^{h(w) k_{u}+1, k_{v}-1, k_{w}-} \text { for } n=3, \\
& \mathcal{T}_{i c d}^{h(w) k_{u} k_{v} k_{w}}=\mathcal{T}_{i c d}^{h(w) k_{u} k_{v} k_{w}+} \bigcup \mathcal{T}_{i c d}^{h(w) k_{u} k_{v}, k_{w}-} \text { for } n=4 .
\end{aligned}
$$

Then

$$
\mathcal{T}_{i}^{h}=\bigcup_{k_{w}=-\infty}^{+\infty} \bigcup_{k_{v}=-\infty}^{+\infty} \bigcup_{k_{u}=-\infty}^{+\infty}\left(\mathcal{T}_{i a b}^{h(w) k_{u} k_{v} k_{w}} \bigcup \mathcal{T}_{i c d}^{h(w) k_{u} k_{v} k_{w}}\right)
$$

and therefore, $\mathcal{T}_{i}^{h}$ consists of a lattice of surfaces $\mathcal{T}_{i a b}^{h(w) k_{u} k_{v} k_{w}}$ and a lattice of surfaces $\mathcal{T}_{i c d}^{h(w) k_{u} k_{v} k_{w}}$, each of which may be considered as formed from the constant frequency curves
Fig. 17 An open cap for the system given in Example 9.5

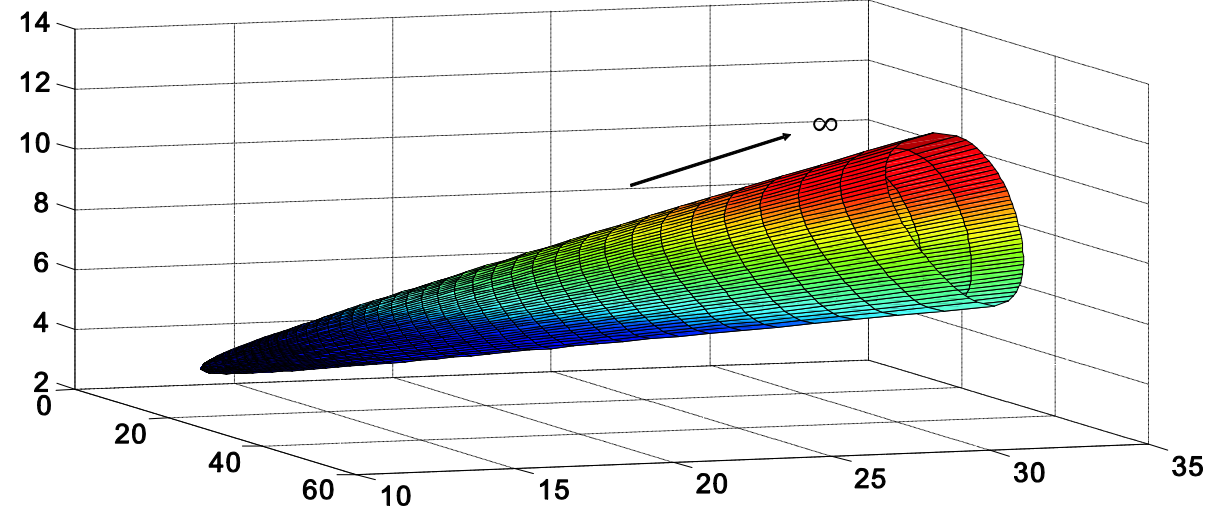




$$
\begin{aligned}
\mathcal{T}_{i a b}^{h(w) k_{u} k_{v} k_{w}}= & \bigcup_{\omega \in \Omega_{i}^{h}} \mathcal{T}_{\omega,\left[\tau_{w}^{a}+2 k_{w} \pi / \omega, \tau_{w}^{b}+2 k_{w} \pi / \omega\right]}^{(w) k_{u} k_{v}}, \\
\mathcal{T}_{i c d}^{h(w) k_{u} k_{v} k_{w}}= & \bigcup_{\omega \in \Omega_{i}^{h}} \mathcal{T}_{\omega,\left[\tau_{w}^{c}+2 k_{w} \pi / \omega, \tau_{w}^{d}+2 k_{w} \pi / \omega\right]}^{(w) k_{u} k_{v}},
\end{aligned}
$$

where $\mathcal{T}_{\omega,\left[\tau_{w}^{a}, \tau_{w}^{b}\right]}^{(w) k_{k} k_{v}}$ and $\mathcal{T}_{\omega,\left[\tau_{w}^{c}, \tau_{w}^{d}\right]}^{(w) k_{u} k_{v}}$ are defined by (6.3), (6.5), (6.6) or (6.7) depending on $m$ and $n$. The specific geometry may be decided with the additional information on the left and right neighbors. The left neighbor is either $\omega_{i}^{h l}=0$, or $\Omega_{i}^{h-1} \subset \Omega_{N}^{k}, k \in\{m, n\}$, and the right neighbor is $\Omega_{i}^{h+1} \subset$ $\Omega_{N}^{l}, l \in\{m, n\}$.

Case ii.a) $\Omega_{i}^{h-1} \subset \Omega_{N}^{k}, \Omega_{i}^{h+1} \subset \Omega_{N}^{k}$.

Type invariance (Corollary 5.2) requires $k \in\{m, n\}$. Without loss of generality, assume $k=n$ (recall $\Omega_{M}^{m n}=$ $\Omega_{M}^{n m}$ ). Then as $\omega \uparrow \omega_{i}^{h r}$ or $\downarrow \omega_{i}^{h l}, M_{l}(\omega) \rightarrow 0, \tau_{w}^{a} \rightarrow$ $\tau_{w}^{L M}+k^{\prime} 2 \pi / \omega, \tau_{w}^{b} \rightarrow \tau_{w}^{L M}+k^{\prime} 2 \pi / \omega$, and each curve $\mathcal{T}_{\omega,\left[\tau_{w}^{a}, \tau_{w}^{b}\right]}^{(w) k_{u} k_{v} k_{w}}$ reduces to one point. Therefore, $\mathcal{T}_{i a b}^{h(w) k_{u} k_{v} k_{w}}$ is a closed surface. On the other hand, $\mathcal{T}_{i c d}^{h(w) k_{u} k_{v} k_{w}}$ is a connector as $\mathcal{T}_{\omega,\left[\tau_{w}^{c}, \tau_{w}^{d}\right]}^{(w) k_{u} k_{v} k_{w}}$ remains a closed curve as $\omega \uparrow \omega_{i}^{h r}$ or $\downarrow \omega_{i}^{h l}$. Indeed, the maximal $\tau_{w}$-interval $\left[\tau_{w}^{c}, \tau_{w}^{d}\right]$ for $\omega \in \Omega_{i}^{h}$ may be considered as continuation of of $\left[\tau_{w}^{a}+k^{\prime \prime} 2 \pi / \omega, \tau_{w}^{b}+\right.$ $\left.k^{\prime \prime} 2 \pi / \omega\right]$ for $\omega \in \Omega_{i}^{h-1}$ as $\omega$ passes through $\omega_{i}^{h l}$, and the situation is similar as $\omega$ passes through $\omega_{i}^{h r}$. Therefore, the connector $\mathcal{T}_{i c d}^{h(w) k_{u} k_{v} k_{w}}$ fits a hole in $\mathcal{T}_{i}^{h-1}$ and another hole in $\mathcal{T}_{i}^{h+1}$.

Case ii.b) $\Omega_{i}^{h-1} \subset \Omega_{N}^{k}, \Omega_{i}^{h+1} \subset \Omega_{N}^{l}, k \neq l$.

Type invariance (Corollary 5.2) requires $k, l \in\{m, n\}$. Without loss of generality, assume $k=m, l=n$. Then, as $\omega \downarrow \omega_{i}^{h l}, M_{l}(\omega) \rightarrow 0, \tau_{w}^{c} \rightarrow \tau_{w}^{L M}+k^{\prime} 2 \pi / \omega, \tau_{w}^{d} \rightarrow$ $\tau_{w}^{L M}+k^{\prime} 2 \pi / \omega$, and $\mathcal{T}_{\omega,\left[\tau_{w}^{c}, \tau_{w}^{d}\right]}^{(w) k_{u} k_{v} k_{w}}$ reduces to one point, while $\mathcal{T}_{\omega,\left[\tau_{w}^{a}, \tau_{w}^{b}\right]}^{(w) k_{u} k_{v} k_{w}}$ remains a closed curve, and $\left[\tau_{w}^{a}, \tau_{w}^{b}\right]$ in $\Omega_{i}^{h}$ may be considered as a continuation of $\left[\tau_{w}^{a}+k^{\prime \prime} 2 \pi / \omega, \tau_{w}^{b}+k^{\prime \prime} 2 \pi / \omega\right]$ in $\Omega_{i}^{h-1}$. Similarly, as $\omega \uparrow \omega_{i}^{h r}, M_{l}(\omega) \rightarrow 0, \tau_{w}^{a} \rightarrow$ $\tau_{w}^{L M}+k^{\prime} 2 \pi / \omega, \tau_{w}^{b} \rightarrow \tau_{w}^{L M}+k^{\prime} 2 \pi / \omega$, and $\mathcal{T}_{\omega,\left[\tau_{w}^{a}, \tau_{w}^{b}\right]}^{(w) k_{u} k_{v} k_{w}}$ reduces to one point, while $\mathcal{T}_{\omega,\left[\tau_{w}^{c}, \tau_{w}^{d}\right]}^{(w) k_{w} k_{v} k_{w}}$ remains a closed curve, and $\left[\tau_{w}^{c}, \tau_{w}^{d}\right]$ in $\Omega_{i}^{h}$ may be considered as a continuation of $\left[\tau_{w}^{a}+k^{\prime \prime} 2 \pi / \omega, \tau_{w}^{b}+k^{\prime \prime} 2 \pi / \omega\right]$ in $\Omega_{i}^{h+1}$. Therefore, $\mathcal{T}_{i a b}^{h(w) k_{u} k_{v} k_{w}}$ is a cap with one end closed, and the other end fits a hole in $\mathcal{T}_{i}^{h-1}$, and $\mathcal{T}_{i c d}^{h(w) k_{u} k_{v} k_{w}}$ is a cap with one end closed, and the other end fits a hole in $\mathcal{T}_{i}{ }^{h+1}$.

Case ii.c) $\omega_{i}^{h l}=0, \Omega_{i}^{h+1} \subset \Omega_{N}^{l}$.

Type invariance (Corollary 5.2) requires $l \in\{m, n\}$. Without loss of generality, assume $l=m$. Then as $\omega \uparrow \omega_{i}^{h r}$, $M_{l}(\omega) \rightarrow 0, \tau_{w}^{c} \rightarrow \tau_{w}^{L M}+k^{\prime} 2 \pi / \omega, \tau_{w}^{d} \rightarrow \tau_{w}^{L M}+k^{\prime} 2 \pi / \omega$, and $\mathcal{T}_{\omega,\left[\tau_{w}^{c}, \tau_{w}^{d}\right]}^{(w) k_{u} k_{v} k_{w}}$ reduces to one point, and $\mathcal{T}_{\omega,\left[\tau_{w}^{a}, \tau_{w}^{b}\right]}^{(w) k_{u} k_{v} k_{w}}$ remains a closed curve. As $\omega \downarrow \omega_{i}^{h l}=0$, both $\mathcal{T}_{\omega,\left[\tau_{w}^{a}, \tau_{w}^{b}\right]}^{(w) k_{u} k_{v} k_{w}}$ and $\mathcal{T}_{\omega,\left[\tau_{w}^{c}, \tau_{w}^{d}\right]}^{(w) k_{u} k_{v} k_{w}}$ approach $\infty$. Therefore, $\mathcal{T}_{i a b}^{h(w) k_{u} k_{v} k_{w}}$ is a semi- open pipe with one end fits a hole of $\mathcal{T}_{i}^{h+1}$ and the other end approaches $\infty$, and $\mathcal{T}_{i c d}^{h(w) k_{u} k_{v} k_{w}}$ is an open cap with one end closed and the other end approaches $\infty$.

Case iii) $\Omega_{i}^{h} \subset \Omega_{G}^{\tau}$.

In this case, each piece of $\mathcal{T}_{i}^{h}$ consists of spirals with continuously varying pitch and a common axis. Such a piece of surface is known as a spiral-like strip as defined below.

Definition 9.6 Let $\mathbf{C}: \mathbb{R} \times \mathbb{R}_{+} \rightarrow \mathbb{R}^{3}$ be continuous, and $\mathbf{C}(\cdot, \omega)$ is a spiral with a common axis $\boldsymbol{\tau}$ for any $\omega \in\left[\omega_{a}, \omega_{b}\right]$. Then

$\mathcal{T}_{\left[\omega_{a}, \omega_{b}\right]}=\left\{\mathbf{C}(\tau, \omega) \mid \tau \in \mathbb{R}, \omega \in\left[\omega_{a}, \omega_{b}\right]\right\}$

is known as a spiral-like strip with axis $\boldsymbol{\tau}$.

Intrinsically, whether $\Omega_{i}^{h} \subset \Omega_{G 1}$ or $\Omega_{i}^{h} \subset \Omega_{G 2}$, there is no difference in terms of the geometric characteristics of the corresponding $\mathcal{T}_{i}^{h}$. Similar to the case of constant frequency curves in the form of spirals, such $\mathcal{T}_{i}^{h}$ is most conveniently parameterized by $\left(\omega, \tau_{w}\right)$ with $\boldsymbol{\tau}_{w}^{0} \cdot \boldsymbol{\tau} \neq 0$, in which case, $\Omega_{i}^{h} \subset \Omega_{G 1}$. When $\boldsymbol{\tau}_{w}^{0} \cdot \boldsymbol{\tau}=0, \Omega_{i}^{h} \subset \Omega_{G 2}$. In the following, both cases will be discussed.

Case iii.1) $\Omega_{i}^{h} \subset \Omega_{G 1}$

Recall in Sect. 6, that a constant frequency curve corresponding to $\omega$ with $\tau_{w}$-maximal interval $(-\infty,+\infty)$ can be described as

$\mathcal{T}_{\omega,(-\infty,+\infty)}^{(w) k_{u} k_{v} \pm}=\left\{\begin{array}{l|l}\left(\tau_{1}, \tau_{2}, \tau_{3}\right) & \begin{array}{l}\tau_{w} \in(-\infty,+\infty) \\ \tau_{u}=\tau_{u}^{k_{u} \pm}\left(\omega, \tau_{w}\right) \\ \tau_{v}=\tau_{v}^{k_{v} \pm}\left(\omega, \tau_{w}\right)\end{array}\end{array}\right\}$.

This is a spiral with axis

$\boldsymbol{\tau}=\delta_{t_{w}}\left(P_{u}^{(w)}(\omega, \cdot)\right) \boldsymbol{\tau}_{u}^{0}+\delta_{t_{w}}\left(P_{v}^{(w)}(\omega, \cdot)\right) \boldsymbol{\tau}_{v}^{0}+\boldsymbol{\tau}_{w}^{0}$.

According to Proposition 8.1, $\boldsymbol{\tau}$ remains constant for for $\omega \in$ $\Omega_{i}^{h}$ although the pitch varies continuously with $\omega$. Therefore, the surface

$$
\mathcal{T}_{i}^{h(w) k_{u} k_{v} \pm}=\overline{\bigcup_{\omega \in \Omega_{i}^{h}} \mathcal{T}_{\omega,(-\infty,+\infty)}^{(w) k_{u} k_{v} \pm}}
$$

for a given $k_{u}, k_{v}$ and + or - sign is a spiral-like strip with axis $\boldsymbol{\tau}$. These spiral-like strips are connected in various ways to form larger surfaces according to the neighbors of $\Omega_{i}^{h}$ to be described below.

Case iii.1.a). $\Omega_{i}^{h} \subset \Omega_{G 1}^{\tau}, \Omega_{i}^{h-1} \subset \Omega_{N}^{k}, \Omega_{i}^{h+1} \subset \Omega_{N}^{k}$.

In this case, for given $k_{u}, k_{v}$ and $k_{w}$ the two patches $\mathcal{T}_{i}^{h(w) k_{u} k_{v}+}$ (corresponding to $\Omega_{i}^{h}$ ) and $\mathcal{T}_{i}^{h+1,(w) k_{u} k_{v} k_{w}+}$ (corresponding to $\Omega_{i}^{h+1}$ ) is a continuous piece of surface parameterized by

$$
\left\{\begin{array}{l|l}
\left(\omega, \tau_{w}\right) & \begin{array}{l}
\tau_{w} \in(-\infty,+\infty) \quad \text { for } \omega \in \Omega_{i}^{h} \\
\tau_{w} \in\left[\tau_{w}^{a}(\omega), \tau_{w}^{b}(\omega)\right] \text { for } \omega \in \Omega_{i}^{h+1}
\end{array}
\end{array}\right\} .
$$


Fig. 18 A pipe

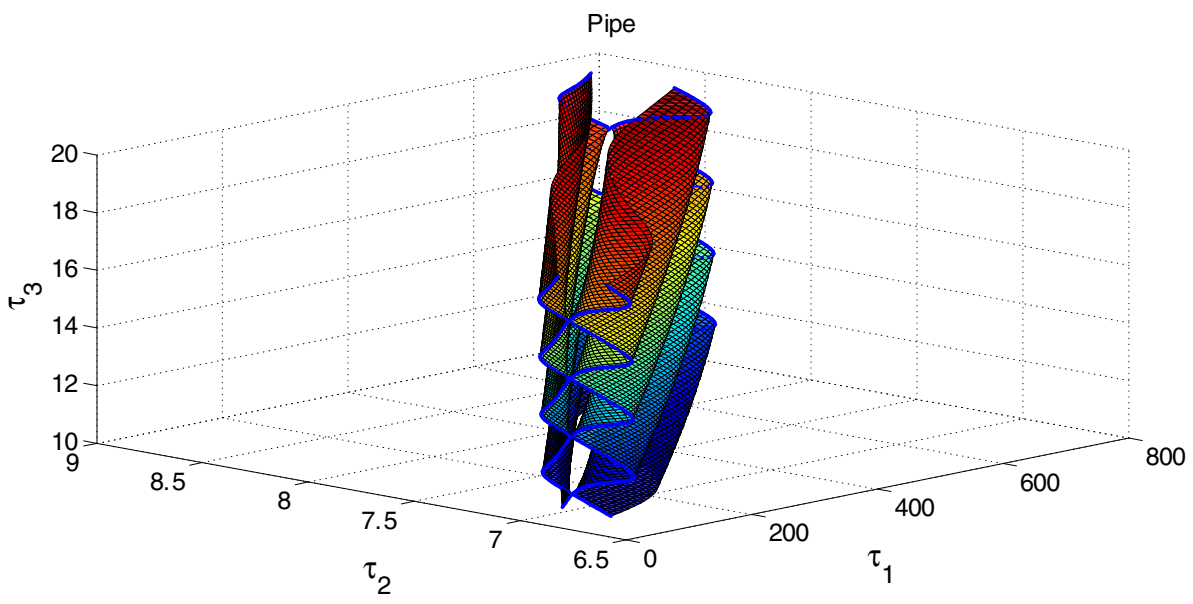

Same can be said about $\mathcal{T}_{i}^{h(w) k_{u} k_{v}-}$ and $\mathcal{T}_{i}^{h+1,(w) k_{u} k_{v} k_{w}-}$.

Consider the case of $k=1$. Then from the discussion about case ia) after Proposition 6.4, it can be easily seen that $\mathcal{T}_{i}^{h+1,(w) k_{u} k_{v} k_{w}+}$ and $\mathcal{T}_{i}^{h+1,(w) k_{u}, k_{v}-1, k_{w}-}$ form a single surface. As $\omega \downarrow \omega_{i}^{h+1, l}=\omega_{i}^{h r}$, the maximal $\tau_{w}$-intervals $\left[\tau_{w}^{a}(\omega), \tau_{w}^{b}(\omega)\right]$ and $\left[\tau_{w}^{a}(\omega)+2 \pi / \omega, \tau_{w}^{b}(\omega)+2 \pi / \omega\right]$ merge, i.e., $\tau_{w}^{b}(\omega)-\tau_{w}^{a}(\omega) \uparrow 2 \pi / \omega_{i}^{h r}$. Define

$\tau_{w}^{a}\left(\omega_{i}^{h r}\right)=\lim _{\omega \downarrow \omega_{i}^{h r}} \tau_{w}^{a}(\omega)$

Then, from continuity and periodicity, we may conclude easily that $\mathcal{T}_{i}^{h(w) k_{u} k_{v}+}$ and $\mathcal{T}_{i}^{h(w) k_{u}, k_{v}-1,-}$ are connected at $\left(\omega_{i}^{h r}, \tau_{w}^{a}\left(\omega_{i}^{h r}\right)+2 \pi k_{w} / \omega_{i}^{h r}\right)$ for $k_{w}=0, \pm 1, \pm 2, \ldots$ Between the connecting points are a series of holes formed by

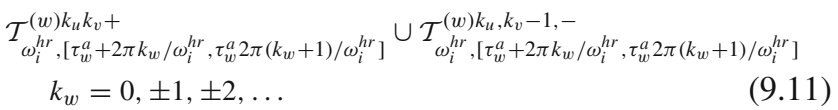

Similarly, we can conclude that $\mathcal{T}_{i}^{h(w) k_{u} k_{v}+}$ and $\mathcal{T}_{i}^{h(w) k_{u}, k_{v}-1,-}$ are also connected at $\left(\omega_{i}^{h l}, \tau_{w}^{a}\left(\omega_{i}^{h l}\right)+2 \pi k_{w} /\right.$ $\left.\omega_{i}^{h l}\right)$, and a series of holes are formed

$$
\begin{aligned}
& \mathcal{T}_{\omega_{i}^{h l},\left[\tau_{w}^{a}+2 \pi k_{w} / \omega_{i}^{h l}, \tau_{w}^{a} 2 \pi\left(k_{w}+1\right) / \omega_{i}^{h l}\right]}^{(w) k_{v}} \cup \mathcal{T}_{\omega_{i}^{h l},\left[\tau_{w}^{a}+2 \pi k_{w} / \omega_{i}^{h l}, \tau_{w}^{a} 2 \pi\left(k_{w}+1\right) / \omega_{i}^{h l}\right]}^{(1), k_{v}-1,-} \\
& \quad k_{w}=0, \pm 1, \pm 2, \ldots
\end{aligned}
$$

where

$\tau_{w}^{a}=\tau_{w}^{a}\left(\omega_{i}^{h l}\right)=\lim _{\omega \uparrow \omega_{i}^{h l}} \tau_{w}^{a}(\omega)$

Therefore, in this case, the two patches $\mathcal{T}_{i}^{h(w) k_{u} k_{v}+}$ and $\mathcal{T}_{i}^{h(w) k_{u}, k_{v}-1,-}$ form a pipe

$\mathcal{T}_{i}^{h(w) k_{u} k_{v}}=\mathcal{T}_{i}^{h(w) k_{u} k_{v}+} \cup \mathcal{T}_{i}^{h(w) k_{u}, k_{v}-1,-}$, if $k=1$

with axis along the $\tau$ direction and two series of holes represented by (9.11) and (9.12).
Analogous analysis allows us to conclude the following: $\mathcal{T}_{i}^{h(w) k_{u} k_{v}}=\mathcal{T}_{i}^{h(w) k_{u} k_{v}+} \cup \mathcal{T}_{i}^{h(w) k_{u}+1, k_{v},-}$, If $k=2$, $\mathcal{T}_{i}^{h(w) k_{u} k_{v}}=\mathcal{T}_{i}^{h(w) k_{u} k_{v}+} \cup \mathcal{T}_{i}^{h(w) k_{u}+1, k_{v}-1,-}$, if $k=3$, $\mathcal{T}_{i}^{h(w) k_{u} k_{v}}=\mathcal{T}_{i}^{h(w) k_{u} k_{v}+} \cup \mathcal{T}_{i}^{h(w) k_{u} k_{v}-}$, if $k=4$.

In all case, $\mathcal{T}_{i}^{h(w) k_{u} k_{v}}$ is a pipe that contains two series of holes with axis $\boldsymbol{\tau}$, and $\mathcal{T}_{i}^{h}$ consists an array of such pipes

$\mathcal{T}_{i}^{h}=\bigcup_{k_{u}=-\infty}^{+\infty} \bigcup_{k_{v}=-\infty}^{+\infty} \mathcal{T}_{i}^{h(w) k_{u} k_{v}}$

Example 9.7 Consider $\Omega_{2}^{2}=\left(\omega_{10}, \omega_{11}\right)$ in the system given in Example 7.4 with For $u=1, v=2$ and $w=3$. It can be verified that $\Omega_{2}^{2}$ is of type $G_{33}^{\boldsymbol{\tau}}$, where $\boldsymbol{\tau}=\boldsymbol{\tau}_{3}$ as $\delta_{\tau_{3}}\left(P_{1}^{(3)}(\omega, \cdot)=0, \delta_{\tau_{3}}\left(P_{2}^{(3)}(\omega, \cdot)=0\right.\right.$. Therefore, $\mathcal{T}_{2}^{2}$ consists of an array of pipes with axes all along $\tau_{3}$, one of these types is shown in Fig. 18.

Case iii.1.b). $\Omega_{i}^{h} \subset \Omega_{G 1}^{\tau}, \Omega_{i}^{h-1} \subset \Omega_{N}^{k}, \Omega_{i}^{h+1} \subset \Omega_{N}^{l}$, $k \neq l$.

Using the same approach as Case iii.1.a), we can obtain the connection patterns, and conclude that they form a wavy sheets with holes. The list of possibilities are listed below.

$k=1, l=2$. In this case, $\mathcal{T}_{i}^{h(w) k_{u} k_{v}+}$ is connected with $\mathcal{T}_{i}^{h(w) k_{u}, k_{v}-1,-}$ at $\omega \downarrow \omega_{i}^{h l}$, which in turns is connected with $\mathcal{T}_{i}^{h(w), k_{u}-1, k_{v}-1,+}$ at $\omega \uparrow \omega_{i}^{h r}$, and so on. Therefore,

$\mathcal{T}_{i}^{h(w) k_{u v}}=\bigcup_{k=-\infty}^{+\infty}\left(\mathcal{T}_{i}^{h(w), k_{u v}+k, k,+} \cup \mathcal{T}_{i}^{h(w), k_{u v}+k, k-1,-}\right)$

are connected with a series of holes between two neighboring patches. This forms a wavy sheet, which roughly parallels a plane with the normal $\boldsymbol{\tau} \times\left(\boldsymbol{\tau}_{u}^{0}+\boldsymbol{\tau}_{v}^{0}\right)$. Each wavy sheet contains a series of holes. 
$k=1, l=3$. In this case, $\mathcal{T}_{i}^{h(w) k_{u} k_{v}+}$ connects with $\mathcal{T}_{i}^{h(w) k_{u}, k_{v}-1,-}$ at $\omega_{i}^{h l}$, which in turn connects with $\mathcal{T}_{i}^{h(w), k_{u}-1, k_{v},+}$ at $\omega_{i}^{h r}$, and so on. This forms the wavy sheet

$\mathcal{T}_{i}^{h(w) k_{u v}}=\bigcup_{k=-\infty}^{+\infty}\left(\mathcal{T}_{i}^{h(w) k k_{u v}+} \cup \mathcal{T}_{i}^{h(w), k, k_{u v}-1,-}\right)$,

which roughly parallels the plane with the normal $\boldsymbol{\tau} \times \boldsymbol{\tau}_{u}^{0}$ and contains an array of holes.

$k=1, l=4$. In this case, the connection is $\mathcal{T}_{i}^{h(w) k_{u} k_{v}+}$ to $\mathcal{T}_{i}^{h(w) k_{u}, k_{v}-1,-}$, and then to $\mathcal{T}_{i}^{h(w) k_{u}, k_{v}-1,+}$, and so on. Therefore, the wavy sheet expression is

$\mathcal{T}_{i}^{h(w) k_{u v}}=\bigcup_{k=-\infty}^{+\infty}\left(\mathcal{T}_{i}^{h(w) k_{u v}, k+} \cup \mathcal{T}_{i}^{h(w), k_{u v}, k-1,-}\right)$

which roughly parallels with the plane with normal $\boldsymbol{\tau} \times \boldsymbol{\tau}_{v}^{0}$ and contains an array of holes.

$k=2, l=3$. In this case, the connection is $\mathcal{T}_{i}^{h(w) k_{u} k_{v}+}$ to $\mathcal{T}_{i}^{h(w) k_{u}+1, k_{v},-}$, and then to $\mathcal{T}_{i}^{h(w) k_{u}, k_{v}+1,+}$, and so on. Therefore, the wavy sheet expression is

$\mathcal{T}_{i}^{h(w) k_{u v}}=\bigcup_{k=-\infty}^{+\infty}\left(\mathcal{T}_{i}^{h(w) k_{u v} k+} \cup \mathcal{T}_{i}^{h(w), k_{u v}+1, k,-}\right)$

which roughly parallels to the plane with normal $\boldsymbol{\tau} \times \boldsymbol{\tau}_{v}^{0}$ and contains an array of holes.

$k=2, l=4$. In this case, the connection is $\mathcal{T}_{i}^{h(w) k_{u} k_{v}+}$ to $\mathcal{T}_{i}^{h(w) k_{u}+1, k_{v},-}$, and then to $\mathcal{T}_{i}^{h(w) k_{u}+1, k_{v},+}$, and so on. Therefore, the wavy sheet expression is

$\mathcal{T}_{i}^{h(w) k_{u v}}=\bigcup_{k=-\infty}^{+\infty}\left(\mathcal{T}_{i}^{h(w) k, k_{u v}+} \cup \mathcal{T}_{i}^{h(w), k+1, k_{u v},-}\right)$,

which roughly parallels to the plane with normal $\boldsymbol{\tau} \times \boldsymbol{\tau}_{u}^{0}$ and contains an array of holes.

$k=3, l=4$. In this case, the connection is $\mathcal{T}_{i}^{h(w) k_{u} k_{v}+}$ to $\mathcal{T}_{i}^{h(w) k_{u}+1, k_{v}-1,-}$, then to $\mathcal{T}_{i}^{h(w) k_{u}+1, k_{v}-1,+}$, and so on. This gives the wavy sheet expression

$$
\begin{aligned}
& \mathcal{T}_{i}^{h(w) k_{u v}}=\bigcup_{k=-\infty}^{+\infty} \\
& \quad \times\left(\mathcal{T}_{i}^{h(w), k_{u v}+k, k_{u v}-k,+} \cup \mathcal{T}_{i}^{h(w), k_{u v}+k+1, k_{u v}-k-1,-}\right),
\end{aligned}
$$

which roughly parallels to plane with normal $\boldsymbol{\tau} \times\left(\boldsymbol{\tau}_{u}^{0}-\boldsymbol{\tau}_{v}^{0}\right)$ and contains an array of holes.
In all these cases, $\mathcal{T}_{i}^{h}$ consists of a series of wavy sheets

$\mathcal{T}_{i}^{h}=\bigcup_{k_{u v}=-\infty}^{+\infty} \mathcal{T}_{i}^{h(w) k_{u v}}$

Case iii.1.c) $\Omega_{i}^{h} \subset \Omega_{G 1}^{\tau}, \omega_{i}^{h l}=0, \Omega_{i}^{h+1} \subset \Omega_{N}^{l}$

For $l=1$,

$\mathcal{T}_{i}^{h(w) k_{u} k_{v}}=\mathcal{T}_{i}^{h(w) k_{u} k_{v}+} \cup \mathcal{T}_{i}^{h(w) k_{u}, k_{v}-1,-}$,

form an open surface. These two surfaces are connected at a series of points corresponding to $\omega \uparrow \omega_{i}^{h r}$, and contains a series of holes. As $\omega \downarrow \omega_{i}^{h l}=0$, both $\mathcal{T}_{i}^{h(w) k_{u} k_{v}+}$ and $\mathcal{T}_{i}^{h(w) k_{u}, k_{v}-1,-}$ approache $\infty$. If $l=2$,

$\mathcal{T}_{i}^{h(w) k_{u} k_{v}}=\mathcal{T}_{i}^{h(w) k_{u} k_{v}+} \cup \mathcal{T}_{i}^{h(w) k_{u}+1, k_{v},-}$

form an open surface with holes. If $l=3$,

$\mathcal{T}_{i}^{h(w) k_{u} k_{v}}=\mathcal{T}_{i}^{h(w) k_{u} k_{v}+} \cup \mathcal{T}_{i}^{h(w) k_{u}+1, k_{v}-1,-}$

form an open surface with holes. If $l=4$,

$\mathcal{T}_{i}^{h(w) k_{u} k_{v}}=\mathcal{T}_{i}^{h(w) k_{u} k_{v}+} \cup \mathcal{T}_{i}^{h(w) k_{u} k_{v}-}$

form an open surface with holes. In all cases, $\mathcal{T}_{i}^{h}$ consists an array of such open surfaces

$\mathcal{T}_{i}^{h}=\bigcup_{k_{u}=-\infty}^{+\infty} \bigcup_{k_{v}=-\infty}^{+\infty} \mathcal{T}_{i}^{h(w) k_{u} k_{v}}$

Case iii.2) $\Omega_{i}^{h} \subset \Omega_{G 2}^{\tau}$

In this case, for each $\omega \in \Omega_{i}^{h}$, there are two $\tau_{w}$-maximal intervals $\left[\tau_{w}^{a}, \tau_{w}^{b}\right]$ and $\left[\tau_{w}^{c}, \tau_{w}^{d}\right], \tau_{w}^{a}<\tau_{w}^{b}<\tau_{w}^{c}<\tau_{w}^{d}<$ $\tau_{w}^{a}+2 \pi / \omega$, and

Type $\left(\omega, \tau_{w}^{a}\right)=\operatorname{Type}\left(\omega, \tau_{w}^{d}\right)=m$,

$\operatorname{Type}\left(\omega, \tau_{w}^{b}\right)=\operatorname{Type}\left(\omega, \tau_{w}^{c}\right)=n$,

for some $m \neq n$.

Similar to Case ii) with $\Omega_{i}^{h} \subset \Omega_{M}$, we may define the curves $\mathcal{T}_{\omega,\left[\tau_{w}^{a}, \tau_{w}^{b}\right]}^{(w) k_{v} k_{w}^{ \pm}}$and $\mathcal{T}_{\omega,\left[\tau_{w}^{c}, \tau_{w}^{d}\right]}^{(w) k_{v} k_{v} k_{w} \pm}$ by (9.4), (9.5), (9.6) and (9.7), from which we can define the patches of surface $\mathcal{T}_{i a b}^{h(w) k_{u} k_{v} k_{w} \pm}$ and $\mathcal{T}_{i c d}^{h(w) k_{u} k_{v} k_{w} \pm}$ by (9.8) and (9.9). Similar to the constant frequency curves discussed in case ii) after Proposition 6.4, the patches $\mathcal{T}_{i a b}^{h(w) k_{u} k_{v} k_{w} \pm}$ and $\mathcal{T}_{i c d}^{h(w) k_{u} k_{v} k_{w} \pm}$ are connected to form two arrays of larger patches $\mathcal{T}_{i a b}^{h(w) k_{u v} k_{w}}$ and $\mathcal{T}_{i c d}^{h(w) k_{u v} k_{w}}$ that extend infinitely in the $\boldsymbol{\tau}$ direction and are confined to certain finite range in other directions. The connection pattern and direction $\tau$ are determined by the types $m$ and $n$. In the following, all possible combinations 
of $m$ and $n$ are listed. Without loss of generality, we list only the case of $m<n$. The case of $m>n$ may be deduced by replacing $\left[\tau_{w}^{a}, \tau_{w}^{b}\right]$ and $\left[\tau_{w}^{c}, \tau_{w}^{d}\right]$ with $\left[\tau_{w}^{c}, \tau_{w}^{d}\right]$ and $\left[\tau_{w}^{a}+\right.$ $\left.2 \pi / \omega, \tau_{w}^{b}+2 \pi / \omega\right]$.

a) If $m=1, n=2$, then $\mathcal{T}_{i a b}^{h(w) k_{u} k_{v} k_{w}+}$ is connected with $\mathcal{T}_{i a b}^{h(w) k_{u}, k_{v}-1, k_{w}-}$, which in turn connects with $\mathcal{T}_{i a b}^{h(w) k_{u}-1, k_{v}-1, k_{w}+}$, and so on, which gives the expression

$$
\mathcal{T}_{i a b}^{h(w) k_{u v} k_{w}}=\bigcup_{k=-\infty}^{+\infty}\left(\mathcal{T}_{i a b}^{h(w) k_{u v}+k, k_{v} k_{w}+} \cup \mathcal{T}_{i a b}^{h(w) k_{u v}+k, k-1_{v} k_{w}-}\right) .
$$

Similarly, we can write

$$
\mathcal{T}_{i c d}^{h(w) k_{u v} k_{w}}=\bigcup_{k=-\infty}^{+\infty}\left(\mathcal{T}_{i c d}^{h(w) k_{u v}+k, k_{v} k_{w}+} \cup \mathcal{T}_{i c d}^{h(w) k_{u v}+k, k-1_{v} k_{w}-}\right) .
$$

As will be seen later on, $\mathcal{T}_{i a b}^{h(w) k_{u v} k_{w}}$ and $\mathcal{T}_{i c d}^{h(w) k_{u v} k_{w}}$ are spiral-like strips with axis

$$
\tau=\tau_{u}^{0}+\boldsymbol{\tau}_{v}^{0}
$$

b) If $m=1, n=2$, then the two spiral-like strips are

$$
\begin{aligned}
& \mathcal{T}_{i a b}^{h(w) k_{u v} k_{w}}=\bigcup_{k=-\infty}^{+\infty}\left(\mathcal{T}_{i a b}^{h(w) k, k_{u v}, k_{w}+} \cup \mathcal{T}_{i a b}^{h(w) k, k_{u v}-1, k_{w}-}\right), \\
& \mathcal{T}_{i c d}^{h(w) k_{u v} k_{w}}=\bigcup_{k=-\infty}^{+\infty}\left(\mathcal{T}_{i c d}^{h(w) k, k_{u v}, k_{w}+} \cup \mathcal{T}_{i c d}^{h(w) k, k_{u v}-1, k_{w}-}\right),
\end{aligned}
$$

and the axis is

$$
\boldsymbol{\tau}=\boldsymbol{\tau}_{u}^{0}
$$

c) If $m=1, n=3$, then the two spiral-like strips are

$$
\begin{aligned}
& \mathcal{T}_{i a b}^{h(w) k_{u v} k_{w}}=\bigcup_{k=-\infty}^{+\infty}\left(\mathcal{T}_{i a b}^{h(w) k_{u v} k k_{w}+} \cup \mathcal{T}_{i a b}^{h(w) k_{u v}, k-1, k_{w}-}\right), \\
& \mathcal{T}_{i c d}^{h(w) k_{u v} k_{w}}=\bigcup_{k=-\infty}^{+\infty}\left(\mathcal{T}_{i c d}^{h(w) k_{u v} k k_{w}+} \cup \mathcal{T}_{i c d}^{h(w) k_{u v}, k-1, k_{w}-}\right),
\end{aligned}
$$

and the axis is

$$
\tau=\tau_{v}^{0}
$$

d) If $m=1, n=4$, then the two spiral-like strips are

$$
\mathcal{T}_{i a b}^{h(w) k_{u v} k_{w}}=\bigcup_{k=-\infty}^{+\infty}\left(\mathcal{T}_{i a b}^{h(w) k_{u v} k k_{w}+} \cup \mathcal{T}_{i a b}^{h(w) k_{u v}+1, k, k_{w}-}\right),
$$

$\mathcal{T}_{i c d}^{h(w) k_{u v} k_{w}}=\bigcup_{k=-\infty}^{+\infty}\left(\mathcal{T}_{i c d}^{h(w) k_{u v} k k_{w}+} \cup \mathcal{T}_{i c d}^{h(w) k_{u v}+1, k, k_{w}-}\right)$,

and the axis is

$\tau=\tau_{v}^{0}$

e) If $m=2, n=3$, then

$$
\begin{aligned}
& \mathcal{T}_{i a b}^{h(w) k_{u v} k_{w}}=\bigcup_{k=-\infty}^{+\infty}\left(\mathcal{T}_{i a b}^{h(w) k k_{u v} k_{w}+} \cup \mathcal{T}_{i a b}^{h(w) k+1, k_{u v}, k_{w}-}\right), \\
& \mathcal{T}_{i c d}^{h(w) k_{u v} k_{w}}=\bigcup_{k=-\infty}^{+\infty}\left(\mathcal{T}_{i c d}^{h(w) k k_{u v} k_{w}+} \cup \mathcal{T}_{i c d}^{h(w) k+1, k_{u v}, k_{w}-}\right), \\
& \boldsymbol{\tau}=\boldsymbol{\tau}_{u}^{0} .
\end{aligned}
$$

f) If $m=3, n=4$, then the two spiral-like strips are

$$
\begin{aligned}
& \mathcal{T}_{i a b}^{h(w) k_{u v} k_{w}}=\bigcup_{k=-\infty}^{+\infty} \\
& \quad \times\left(\mathcal{T}_{i a b}^{h(w) k, k_{u v}-k, k_{w}+} \cup \mathcal{T}_{i a b}^{h(w) k+1, k_{u v}-k-1, k_{w}-}\right), \\
& \mathcal{T}_{i c d}^{h(w) k_{u v} k_{w}}=\bigcup_{k=-\infty}^{+\infty} \\
& \quad \times\left(\mathcal{T}_{i c d}^{h(w) k, k_{u v}-k, k_{w}+} \cup \mathcal{T}_{i c d}^{h(w) k+1, k_{u v}-k-1, k_{w}-}\right),
\end{aligned}
$$

and the axis is

$$
\boldsymbol{\tau}=\boldsymbol{\tau}_{u}^{0}-\boldsymbol{\tau}_{v}^{0}
$$

To see that $\mathcal{T}_{i a b}^{h(w) k_{u v} k_{w}}$ and $\mathcal{T}_{i c d}^{h(w) k_{u v} k_{w}}$ are indeed spirallike strips, it can be seen that they can equivalently considered as formed from the spirals (constant frequency curves) discussed in Case ii) after Proposition 6.4,

$$
\begin{aligned}
& \mathcal{T}_{i a b}^{h(w) k_{u v} k_{w}}=\overline{\bigcup_{\omega \in \Omega_{i}^{h}} \mathcal{T}_{\omega,\left[\tau_{w}^{d}+2 \pi k_{w} / \omega, \tau_{w}^{b}+2 \pi k_{w} / \omega\right]}^{(w)},} \\
& \mathcal{T}_{i c d}^{h(w) k_{u v} k_{w}}=\overline{\bigcup_{\omega \in \Omega_{i}^{h}} \mathcal{T}_{\omega,\left[\tau_{w}^{c}+2 \pi k_{w} / \omega, \tau_{w}^{d}+2 \pi k_{w} / \omega\right]}^{(w)} .}
\end{aligned}
$$

The spiral-like strips $\mathcal{T}_{i a b}^{h(w) k_{u v} k_{w}}$ and $\mathcal{T}_{i c d}^{h(w) k_{u v}^{\prime} k_{w}^{\prime}}$ for some $k_{u v}^{\prime}$ and $k_{w}^{\prime}$ are further connected. The connection pattern depends on the neighbors of $\Omega_{i}^{h}$. There are three possible cases.

Case iii.2.a) $\Omega_{i}^{h} \subset \Omega_{G 2}^{\tau}, \Omega_{i}^{h-1} \subset \Omega_{N}^{k}, \Omega_{i}^{h+1} \subset \Omega_{N}^{k}$.

Type invariance (Corollary 5.2) requires $k \in\{m, n\}$. If $k=m$, then as $\omega \uparrow \omega_{i}^{h r}$ or $\downarrow \omega_{i}^{h l}, V_{l} \rightarrow 0$, and $\tau_{w}^{b} \rightarrow \tau_{w}^{L V}$, $\tau_{w}^{c} \rightarrow \tau_{w}^{L V}$, and the two maximal $\tau_{w}$-intervals $\left[\tau_{w}^{a}, \tau_{w}^{b}\right]$ and $\left[\tau_{w}^{c}, \tau_{w}^{d}\right]$ merge to become one interval in $\Omega_{i}^{h-1}$ or $\Omega_{i}^{h+1}$. As 
a result, the two spiral strips $\mathcal{T}_{i a b}^{h(w) k_{u v} k_{w}}$ and $\mathcal{T}_{i c d}^{h(w) k_{u v} k_{w}}$ form a surface

$\mathcal{T}_{i}^{h(w) k_{u v} k_{w}}=\mathcal{T}_{i a b}^{h(w) k_{u v} k_{w}} \cup \mathcal{T}_{i c d}^{h(w) k_{u v} k_{w}}$.

This surface has the shape of a pipe with two series of holes corresponding to $\omega=\omega_{i}^{h l}$ and $\omega_{i}^{h r}$. At $\omega=\omega_{i}^{h l}$, the holes are defined by the two spirals

$\lim _{\omega \downarrow \omega_{i}^{h l}} \mathcal{T}_{\omega,\left[\tau_{w}^{a}+2 \pi k_{w} / \omega, \tau_{w}^{b}+2 \pi k_{w} / \omega\right]}^{(w)}$

and

$\lim _{\omega \downarrow \omega_{i}^{h l}} \mathcal{T}_{\omega,\left[\tau_{w}^{c}+2 \pi k_{w} / \omega, \tau_{w}^{d}+2 \pi k_{w} / \omega\right]}^{(w) k_{u v}}$

in view of the fact that these two spirals touch each other at the parameter $\tau_{w}^{b}=\tau_{w}^{c}$. The holes corresponding to $\omega=\omega_{i}^{h r}$ are similarly formed from two spirals.

Similarly, if $k=m$, then as $\omega \uparrow \omega_{i}^{h r}$ or $\downarrow \omega_{i}^{h l}, V_{l} \rightarrow 0$, and $\tau_{w}^{d} \rightarrow \tau_{w}^{L V}, \tau_{w}^{a}+2 \pi / \omega \rightarrow \tau_{w}^{L V}$, and the two maximal $\tau_{w}$-intervals $\left[\tau_{w}^{c}, \tau_{w}^{d}\right]$ and $\left[\tau_{w}^{a}+2 \pi / \omega, \tau_{w}^{b}+2 \pi / \omega\right]$ merge to become one interval in $\Omega_{i}^{h-1}$ or $\Omega_{i}^{h+1}$. Therefore, $\mathcal{T}_{i c d}^{h(w) k_{u v} k_{w}}$ and $\mathcal{T}_{i a b}^{h(w), k_{u v}, k_{w}+1}$ forms a pipe

$\mathcal{T}_{i}^{h(w) k_{u v} k_{w}}=\mathcal{T}_{i c d}^{h(w) k_{u v} k_{w}} \cup \mathcal{T}_{i a b}^{h(w), k_{u v}, k_{w}+1}$,

with two series of holes formed by the spirals expressed as $\mathcal{T}_{\omega,\left[\tau_{w}^{c}+2 \pi k_{w} / \omega, \tau_{w}^{d}+2 \pi k_{w} / \omega\right]}^{(w) k_{u v}}$ and $\mathcal{T}_{\omega,\left[\tau_{w}^{a}+2 \pi\left(k_{w}+1\right) / \omega, \tau_{w}^{b}+2 \pi\left(k_{w}+1\right) / \omega\right]}^{(w)}$ as $\omega \downarrow \omega_{i}^{h l}$ and $\uparrow \omega_{i}^{h r}$.

Obviously, $\mathcal{T}_{i}^{h}$ consists an array of such pipes,

$\mathcal{T}_{i}^{h}=\bigcup_{k_{u v}=-\infty}^{+\infty} \bigcup_{k_{w}=-\infty}^{+\infty} \mathcal{T}_{i}^{h(w) k_{u v} k_{w}}$

Case iii.2.b) $\Omega_{i}^{h} \subset \Omega_{G 2}^{\tau}, \Omega_{i}^{h-1} \subset \Omega_{N}^{k}, \Omega_{i}^{h+1} \subset \Omega_{N}^{l}$, $k \neq l$.

Type invariance (Corollary 5.2) requires either $k=m$, $l=n$ or $k=n, l=m$.

First consider $k=m, l=n$. In this case, as $\omega \downarrow \omega_{i}^{h l}$, $V_{l} \rightarrow 0$, and $\tau_{w}^{b} \rightarrow \tau_{w}^{L V}, \tau_{w}^{c} \rightarrow \tau_{w}^{L V}$, and the two maximal $\tau_{w}$-intervals $\left[\tau_{w}^{a}, \tau_{w}^{b}\right]$ and $\left[\tau_{w}^{c}, \tau_{w}^{d}\right]$ merge to become one interval in $\Omega_{i}^{h-1}$. Therefore, $\mathcal{T}_{i a b}^{h(w) k_{u v} k_{w}}$ is connected with $\mathcal{T}_{i c d}^{h(w) k_{u v} k_{w}}$ with a series of holes formed by the two spirals corresponding to $\omega_{i}^{h l}$. As $\omega \uparrow \omega_{i}^{h r}, V_{l} \rightarrow 0$, and $\tau_{w}^{d} \rightarrow \tau_{w}^{L V}, \tau_{w}^{a}+2 \pi / \omega \rightarrow \tau_{w}^{L V}$, and the two maximal $\tau_{w}$-intervals $\left[\tau_{w}^{c}, \tau_{w}^{d}\right]$ and $\left[\tau_{w}^{a}+2 \pi / \omega, \tau_{w}^{b}+2 \pi / \omega\right]$ merge to become one interval in $\Omega_{i}^{h+1}$, and $\mathcal{T}_{i c d}^{h(w) k_{u v} k_{w}}$ is connected with $\mathcal{T}_{i a b}^{h(w), k_{u v}, k_{w}+1}$ with a series of holes formed by two spirals corresponding to $\omega_{i}^{h r}$. Considering $\omega \downarrow \omega_{i}^{h l}$ again, we may conclude that $\mathcal{T}_{i a b}^{h(w), k_{u v}, k_{w}+1}$ is connected with $\mathcal{T}_{i c d}^{h(w), k_{u v}, k_{w}+1}$. Continue this process, we realize that

$\mathcal{T}_{i}^{h(w) k_{u v}}=\bigcup_{k_{w}=-\infty}^{+\infty}\left(\mathcal{T}_{i a b}^{h(w) k_{u v} k_{w}} \cup \mathcal{T}_{i c d}^{h(w) k_{u v} k_{w}}\right)$

is a wavy sheet. This sheet is roughly parallel to the plane with normal of $\boldsymbol{\tau}_{w}^{0} \times \boldsymbol{\tau}$.

For $k=n$ and $l=m$, we may similarly conclude that (9.13) is a wavy sheet. In both cases, $\mathcal{T}_{i}^{h}$ consists of a series of such wavy sheets

$\mathcal{T}_{i}^{h}=\bigcup_{k_{u v}=-\infty}^{+\infty} \mathcal{T}_{i}^{h(w) k_{u v}}$

Case iii.2.c) $\omega_{i}^{h l}=0, \Omega_{i}^{h+1} \subset \Omega_{N}^{l}$.

Consider $\omega \uparrow \omega_{i}^{h r}$. Then for $l=n$, we see that $\mathcal{T}_{i c d}^{h(w) k_{u v} k_{w}}$ and $\mathcal{T}_{i a b}^{h(w), k_{u v}, k_{w}+1}$ are connected to form a surface

$\mathcal{T}_{i}^{h(w) k_{u v} k_{w}}=\mathcal{T}_{i c d}^{h(w) k_{u v} k_{w}} \cup \mathcal{T}_{i a b}^{h(w), k_{u v}, k_{w}+1}$

that contains a series of holes. Otherwise, i.e., $l=m$, then $\mathcal{T}_{i a b}^{h(w) k_{u v} k_{w}}$ and $\mathcal{T}_{i c d}^{h(w) k_{u v} k_{w}}$ are connected to form a surface

$\mathcal{T}_{i}^{h(w) k_{u v} k_{w}}=\mathcal{T}_{i a b}^{h(w) k_{u v} k_{w}} \cup \mathcal{T}_{i c d}^{h(w) k_{u v} k_{w}}$

that contains a series of holes. In either case, as $\omega \downarrow \omega_{i}^{h l}=0$, $\mathcal{T}_{i}^{h(w) k_{u v} k_{w}}$ approaches $\infty$. Therefore, $\mathcal{T}_{i}^{h(w) k_{u v} k_{w}}$ is an open surface that contains a series of holes. Obviously, $\mathcal{T}_{i}^{h}$ consists of an array of such open surfaces

$\mathcal{T}_{i}^{h}=\bigcup_{k_{u v}=-\infty}^{+\infty} \bigcup_{k_{w}=-\infty}^{+\infty} \mathcal{T}_{i}^{h(w) k_{u v} k_{w}}$

\section{The remaining case}

In this section, we will consider the case $\Omega_{i}^{h} \subset \Omega_{M}^{k k}$, which has not been covered in Theorem 9.1.

In this case, Type invariance (Corollary 5.2) requires the right neighbor must be $\Omega_{i}^{h+1} \subset \Omega_{N}^{k}$, and the left neighbor be either $\Omega_{i}^{h-1} \subset \Omega_{N}^{k}$ or $\omega_{i}^{h l}=0$. Similar to Case ii) of Sect. 9, we may form the patches $\mathcal{T}_{i a b}^{h(w) k_{u} k_{v} k_{w}}$ according to $k$,

$$
\begin{aligned}
& \mathcal{T}_{i a b}^{h(w) k_{u} k_{v} k_{w}}=\mathcal{T}_{i a b}^{h(w) k_{u} k_{v} k_{w}+} \bigcup \mathcal{T}_{i a b}^{h(w) k_{u}, k_{v}-1, k_{w}-} \text { for } k=1, \\
& \mathcal{T}_{i a b}^{h(w) k_{u} k_{v} k_{w}}=\mathcal{T}_{i a b}^{h(w) k_{u} k_{v} k_{w}+} \bigcup \mathcal{T}_{i a b}^{h(w) k_{u}+1, k_{v}, k_{w}-} \text { for } k=2 \text {, } \\
& \mathcal{T}_{i a b}^{h(w) k_{u} k_{v} k_{w}}=\mathcal{T}_{i a b}^{h(w) k_{u} k_{v} k_{w}+} \bigcup \mathcal{T}_{i a b}^{h(w) k_{u}+1, k_{v}-1, k_{w}-} \text { for } k=3, \\
& \mathcal{T}_{i a b}^{h(w) k_{u} k_{v} k_{w}}=\mathcal{T}_{i a b}^{h(w) k_{u} k_{v} k_{w}+} \bigcup \mathcal{T}_{i a b}^{h(w) k_{u} k_{v}, k_{w}-} \text { for } k=4,
\end{aligned}
$$


where $\mathcal{T}_{i a b}^{h(w) k_{u} k_{v} k_{w} \pm}$ are defined in (9.8). Equivalently, $\mathcal{T}_{i a b}^{h(w) k_{u} k_{v} k_{w}}$ may be considered as formed by constant frequency curves

$$
\mathcal{T}_{i a b}^{h(w) k_{u} k_{v} k_{w}}=\overline{\bigcup_{\omega \in \Omega_{i}^{h}} \mathcal{T}_{\omega,\left[\tau_{w}^{a}+2 k_{w} \pi / \omega, \tau_{w}^{b}+2 k_{w} \pi / \omega\right]}^{(w) k_{v}}}
$$

These constant frequency curves are closed curves. Similarly, $\mathcal{T}_{i c d}^{h(w) k_{u} k_{v} k_{w}}$ is also a patch formed by closed curves

$$
\mathcal{T}_{i c d}^{h(w) k_{u} k_{v} k_{w}}={\overline{\bigcup_{\omega \in \Omega_{i}^{h}} \mathcal{T}_{\omega,\left[\tau_{w}^{c}+2 k_{w} \pi / \omega, \tau_{w}^{d}+2 k_{w} \pi / \omega\right]}^{(w) k_{v}}}}_{.}^{.}
$$

However, the determination of the geometric form of $\mathcal{T}_{i a b}^{h(w) k_{u} k_{v} k_{w}}$ and $\mathcal{T}_{i c d}^{h(w) k_{u} k_{v} k_{w}}$ may not be completely determined by its neighbors. The additional information needed is whether $V_{l}(\omega)=0$ or $M_{l}(\omega)$ at $\omega=\omega_{i}^{h r}$, and $\omega_{i}^{h l}$ in the case of $\Omega_{i}^{h-1} \subset \Omega_{N}^{k}$. We will consider each case in the following.

Case i) $\omega_{i}^{h l}=0, V_{l}\left(\omega_{i}^{h r}\right)=0$.

In this case, as $\omega \uparrow \omega_{i}^{h r}$, either $\tau_{w}^{c}-\tau_{w}^{b} \longrightarrow 0$, and the two intervals $\left[\tau_{w}^{a}, \tau_{w}^{b}\right]$ and $\left[\tau_{w}^{c}, \tau_{w}^{d}\right]$ merge into a single interval, or $\tau_{w}^{a}+2 \pi / \omega-\tau_{w}^{d} \rightarrow 0$, and the two intervals $\left[\tau_{w}^{c}, \tau_{w}^{d}\right]$ and $\left[\tau_{w}^{a}+2 \pi / \omega, \tau_{w}^{b}+2 \pi / \omega\right]$ merge into a single interval. In either case, $\mathcal{T}_{\omega,\left[\tau_{w}^{a}+2 k_{w} \pi / \omega, \tau_{w}^{b}+2 k_{w} \pi / \omega\right]}^{(w) k_{v}}$ and $\mathcal{T}_{\omega,\left[\tau_{w}^{c}+2 k_{w} \pi / \omega, \tau_{w}^{d}+2 k_{w} \pi / \omega\right]}^{(w) k_{v}}$ remain closed curves as $\omega \uparrow \omega_{i}^{h r}$, although either $\mathcal{T}_{\omega,\left[\tau^{a}+2 k_{w} \pi / \omega, \tau^{b}+2 k_{w} \pi / \omega\right]}^{(w)}$ and $\mathcal{T}_{\omega,\left[\tau_{w}^{c}+2 k_{w} \pi / \omega, \tau_{w}^{d}+2 k_{w} \pi / \omega\right]}^{(w) k_{u} k_{v}}$, or $\mathcal{T}_{\omega,\left[\tau_{w}^{c}+2 k_{w} \pi / \omega, \tau_{w}^{d}+2 k_{w} \pi / \omega\right]}^{(w) k_{u} k_{v}}$ and $\mathcal{T}_{\omega,\left[\tau_{w}^{a}+2\left(k_{w}+1\right) \pi / \omega, \tau_{w}^{b}+2\left(k_{w}+1\right) \pi / \omega\right]}^{(w) k_{v}}$ are on the verge of merging into one single closed curve. As $\omega \downarrow \omega_{i}^{h l}=0$,

$\mathcal{T}_{\omega,\left[\tau_{w}^{a}+2 k_{w} \pi / \omega, \tau_{w}^{b}+2 k_{w} \pi / \omega\right]}^{(w) k_{v}} \quad$ and $\mathcal{T}_{\omega,\left[\tau_{w}^{c}+2 k_{w} \pi / \omega, \tau_{w}^{d}+2 k_{w} \pi / \omega\right]}^{(w) k_{k} k_{v}}$ both approach $\infty$. Therefore, $\mathcal{T}_{i a b}^{h(w) k_{u} k_{v} k_{w}}$ and $\mathcal{T}_{i c d}^{h(w) k_{u} k_{v} k_{w}}$ are semi-open pipes. As a result,

$$
\mathcal{T}_{i}^{h}=\bigcup_{k_{u}=-\infty}^{+\infty} \bigcup_{k_{v}=-\infty}^{+\infty} \bigcup_{k_{w}=-\infty}^{+\infty}\left(\mathcal{T}_{i a b}^{h(w) k_{u} k_{v} k_{w}} \bigcup \mathcal{T}_{i c d}^{h(w) k_{u} k_{v} k_{w}}\right)
$$

is two lattices of semi-open pipes, and pairs of such semiopen pipes are on the verge of merging together on one end.

Case ii) $\omega_{i}^{h l}=0, M_{l}\left(\omega_{i}^{h r}\right)=0$.

In this case, as $\omega \uparrow \omega_{i}^{h r}$, either $\tau_{w}^{b}-\tau_{w}^{a} \rightarrow 0$ and $\mathcal{T}_{\omega,\left[\tau_{w}^{a}+2 k_{w} \pi / \omega, \tau_{w}^{b}+2 k_{w} \pi / \omega\right]}^{(w) k_{v}}$ reduces to one point, or $\tau_{w}^{d}-$ $\tau_{w}^{c} \stackrel{\rightarrow}{ } \rightarrow 0$ and $\mathcal{T}_{\omega,\left[\tau_{w}^{c}+2 k_{w} \pi / \omega, \tau_{w}^{d}+2 k_{w} \pi / \omega\right]}^{(w) k_{v}}$ reduces to one point. As $\omega \downarrow \omega_{i}^{h l}=0, \mathcal{T}_{\omega,\left[\tau_{w}^{a}+2 k_{w} \pi / \omega, \tau_{w}^{b}+2 k_{w} \pi / \omega\right]}^{(w)}$ and $\mathcal{T}_{\omega,\left[\tau_{w}^{c}+2 k_{w} \pi / \omega, \tau_{w}^{d}+2 k_{w} \pi / \omega\right]}^{(w) k_{v}}$ both approach $\infty$. Therefore, one among $\mathcal{T}_{i a b}^{h(w) k_{u} k_{v} k_{w}}$ and $\mathcal{T}_{i c d}^{h(w) k_{u} k_{v} k_{w}}$ is an open cap, the other is semi-open pipe.

Case iii) $V_{l}\left(\omega_{i}^{h l}\right)=0, V_{l}\left(\omega_{i}^{h r}\right)=0$.
In this case, $\mathcal{T}_{\omega,\left[\tau_{w}^{a}+2 k_{w} \pi / \omega, \tau_{w}^{b}+2 k_{w} \pi / \omega\right]}^{(w) k_{v}} \quad$ and $\mathcal{T}_{\omega,\left[\tau_{w}^{c}+2 k_{w} \pi / \omega, \tau_{w}^{d}+2 k_{w} \pi / \omega\right]}$ remain as closed curves as $\omega \uparrow$ $\omega_{i}^{h r}$ and $\downarrow \omega_{i}^{h l}$. Therefore, both $\mathcal{T}_{i a b}^{h(w) k_{u} k_{v} k_{w}}$ and $\mathcal{T}_{i c d}^{h(w) k_{u} k_{v} k_{w}}$ are connectors, although they are on the verge of merging on both ends. The merging could be the same two connectors or two different connectors on the two ends.

Case iv) $V_{l}\left(\omega_{i}^{h l}\right)=0, M_{l}\left(\omega_{i}^{h r}\right)=0$.

In this case, as $\omega \uparrow \omega_{i}^{h r}$, one of the two closed curves $\mathcal{T}^{(w) k_{u} k_{v}}$ $\mathcal{T}_{\omega,\left[\tau_{w}^{a}+2 k_{w} \pi / \omega, \tau_{w}^{b}+2 k_{w} \pi / \omega\right]}$ and $\mathcal{T}_{\omega,\left[\tau_{w}^{c}+2 k_{w} \pi / \omega, \tau_{w}^{d}+2 k_{w} \pi / \omega\right]}^{(w) k_{k} k_{v}}$ reduces to one point, and the other remains closed curve. As $\omega \downarrow \omega_{i}^{h l}$, two closed curves are on the verge of merging together. Therefore, $\mathcal{T}_{i a b}^{h(w) k_{u} k_{v} k_{w}}$ and $\mathcal{T}_{i c d}^{h(w) k_{u} k_{v} k_{w}}$ consist of one cap and one connector. Pairs of cap and connectors are on the verge of merging.

Case v) $M_{l}\left(\omega_{i}^{h l}\right)=0, V_{l}\left(\omega_{i}^{h r}\right)=0$.

This is very similar to Case iv) except on the opposite sides.

Case vi) $M_{l}\left(\omega_{i}^{h l}\right)=0, M_{l}\left(\omega_{i}^{h r}\right)=0$.

Using the similar analysis, we may easily conclude that $\mathcal{T}_{\omega,\left[\tau_{w}^{a}+2 k_{w} \pi / \omega, \tau_{w}^{b}+2 k_{w} \pi / \omega\right]}^{(w) k_{k} k_{v}}$ and $\mathcal{T}_{\omega,\left[\tau_{w}^{c}+2 k_{w} \pi / \omega, \tau_{w}^{d}+2 k_{w} \pi / \omega\right]}^{(w) k_{v} k_{v}}$ either consist of two caps or one connector one closed surface.

Example 10.1 Consider $\Omega_{1}^{2}=\left(\omega_{3}, \omega_{4}\right)$ in the system given in Example 7.4. For $u=1, v=2$ and $w=3$, it can be verified that $\Omega_{1}^{2}$ is of type $M_{11}^{11}$, and $M_{l}\left(\omega_{3}\right)=0, V_{l}\left(\omega_{4}\right)=0$, which is case v) above. Therefore, $\mathcal{T}_{1}^{2}$ consists of one cap and one connector, and they touch each other at $\omega_{4}$ as shown in Fig. 19. As $\omega$ increases beyond $\omega_{4}$, the two curves merge into one. The surfaces in this figure matches the one in Figure 12 without seam.

\section{Stability analysis example}

To illustrate how the above results can be used to conduct stability analysis, we will analyze the system with characteristic equation

$\Delta(s)=0$,

where $\Delta(s)$ is given in (1.1) with

$$
\begin{aligned}
& p_{0}(s)=s^{3}+3 s+7, \\
& p_{1}(s)=s^{2}+3 s+1, \\
& p_{2}(s)=4 s+3, \\
& p_{3}(s)=s^{2}+s+0.1, \\
& p_{12}(s)=1 \\
& p_{23}(s)=0.5 s+0.5, \\
& p_{31}(s)=s+1, \\
& p_{123}(s)=0.5 .
\end{aligned}
$$


Fig. 19 Cap-connector

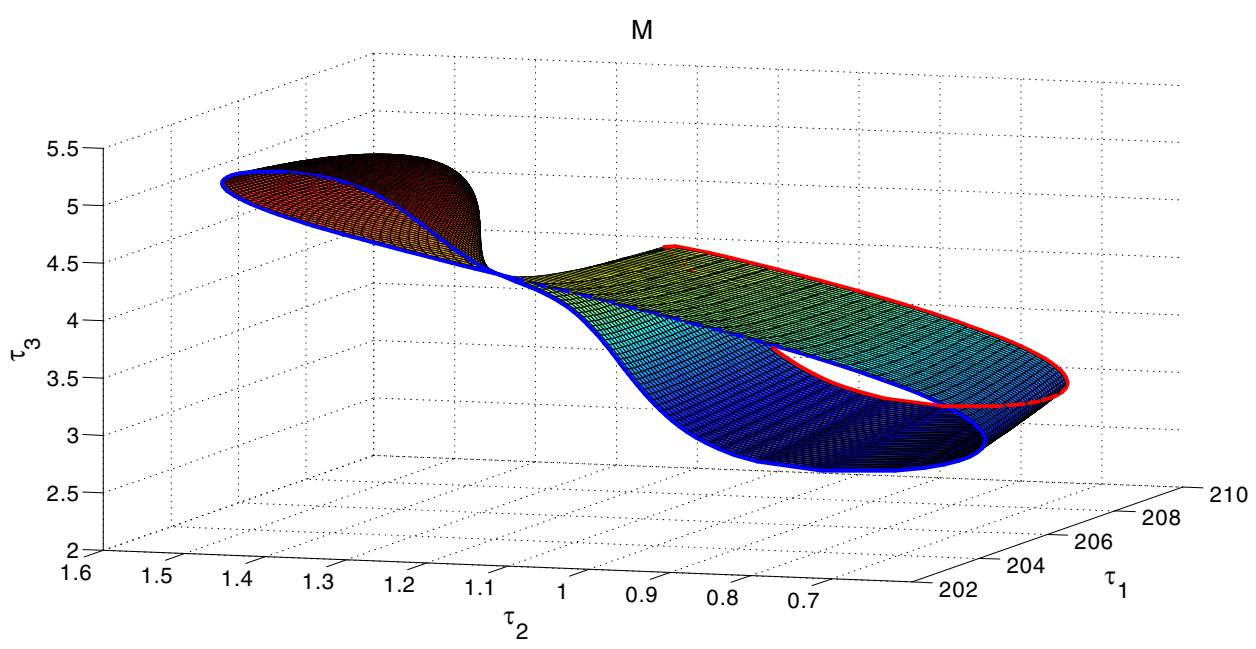

Fig. $20 V_{g}, V_{l}, M_{g}$ and $M_{l}$ vs $\omega$ for the system with coefficients (11.2-11.9)

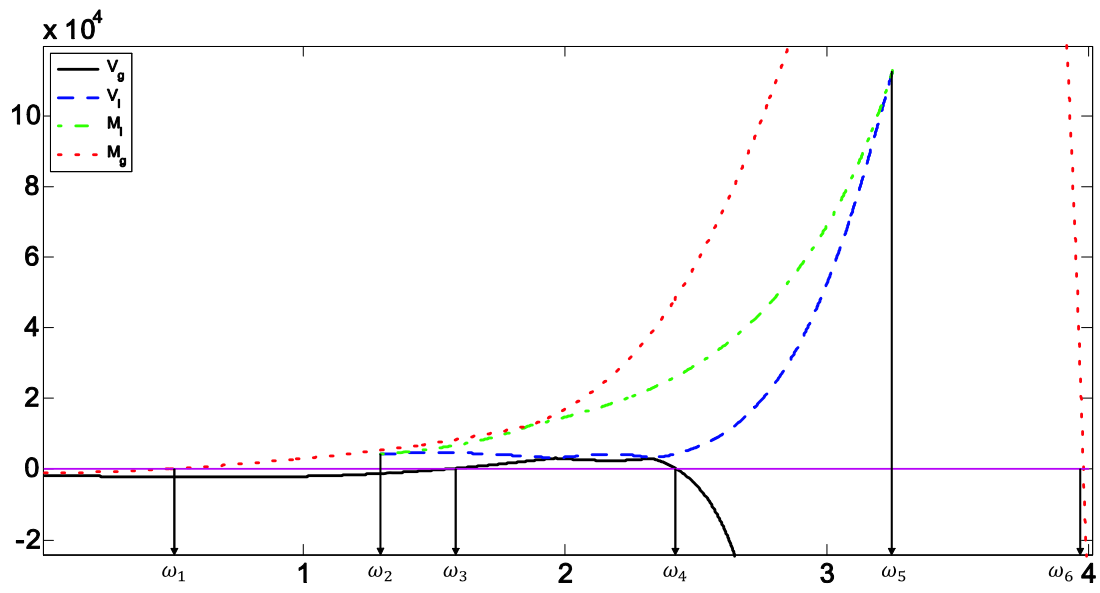

The idea is very similar to Section VII of [22]. Specifically, as $\Delta(s)$ depends on $s$ and $\left(\tau_{1}, \tau_{2}, \tau_{3}\right),(11.1)$ defines $s$ as an implicit function of $\left(\tau_{1}, \tau_{2}, \tau_{3}\right)$. Let $\left(\tau_{1}^{*}, \tau_{2}^{*}, \tau_{3}^{*}\right)$ be a point on a surface of the stability crossing set, and let $s=j \omega^{*}$ satisfy

$$
\Delta\left(j \omega^{*}\right)=0
$$

when the delays are equal to $\left(\tau_{1}^{*}, \tau_{2}^{*}, \tau_{3}^{*}\right)$. Let $\xi=\left(\xi_{1}, \xi_{2}, \xi_{3}\right)$ be a unit vector in the delay parameter space that is not tangent to the surface. Then, if the real part of the directional derivative

$\operatorname{Re}\left[\frac{\partial s}{\partial \xi}\right]_{\substack{\tau_{k}=\tau_{k}^{*}, k=1,2,3 \\ s=j \omega^{*}}}=\operatorname{Re}\left[\sum_{k=1}^{3} \frac{\partial s}{\partial \tau_{k}} \xi_{k}\right]_{\substack{\tau_{k}=\tau_{k}^{*}, k=1,2,3 \\ s=j \omega^{*}}}>0$

then a pair of roots of $\Delta(s)$ cross the imaginary axis from left to the right, thus increasing the right-half-plane roots by two, when the delays $\left(\tau_{1}, \tau_{2}, \tau_{3}\right)$ crosses the surface along the direction of $\xi$. The crossing is in the opposite direction if the inequality in (11.10) is opposite.

Figure 20 shows $V_{g}, V_{l}, M_{g}$ and $M_{l}$ vs $\omega$, from which it can be easily identified that $\left[\omega_{1}, \omega_{2}\right) \subset \Omega_{N 3},\left[\omega_{2}, \omega_{3}\right] \subset \Omega_{N 2}$, $\left(\omega_{3}, \omega_{4}\right) \subset \Omega_{G 1},\left[\omega_{4}, \omega_{5}\right] \subset \Omega_{N 2},\left(\omega_{5}, \omega_{6}\right] \subset \Omega_{N 3}$. It can be checked that the boundaries are all type 3 . Therefore, $\left[\omega_{1}, \omega_{3}\right]$ corresponds to caps, $\left(\omega_{3}, \omega_{4}\right)$ corresponds to pipes, and $\left[\omega_{4}, \omega_{6}\right]$ corresponds to caps, where

$\omega_{1}=0.4642566$,

$\omega_{3}=1.5413434$,

$\omega_{4}=2.4298395$,

$\omega_{6}=3.9809737$.

Therefore, the stability crossing set consists of the cap-pipecap combinations.

Using the method developed in this article, the stability crossing set is parameterized and plotted in Fig. 21. There are some small structures that are not easily seen from this diagram. Using the Routh-Hurwitz criterion, it can be easily calculated that the system is stable when all the delays are set to zero. By considering the crossing direction, it can be shown 
Fig. 21 Stability crossing set for the system with coefficients (11.2-11.9). Only complete cap-pipe-cap combinations within the parameter range are shown

Fig. 22 Cross section of the stability crossing set with $\tau_{3}=0$ for the system with coefficients (11.2-11.9)

Fig. 23 Cross section of the stability crossing set with $\tau_{3}=1$ for the system with coefficients (11.2-11.9)
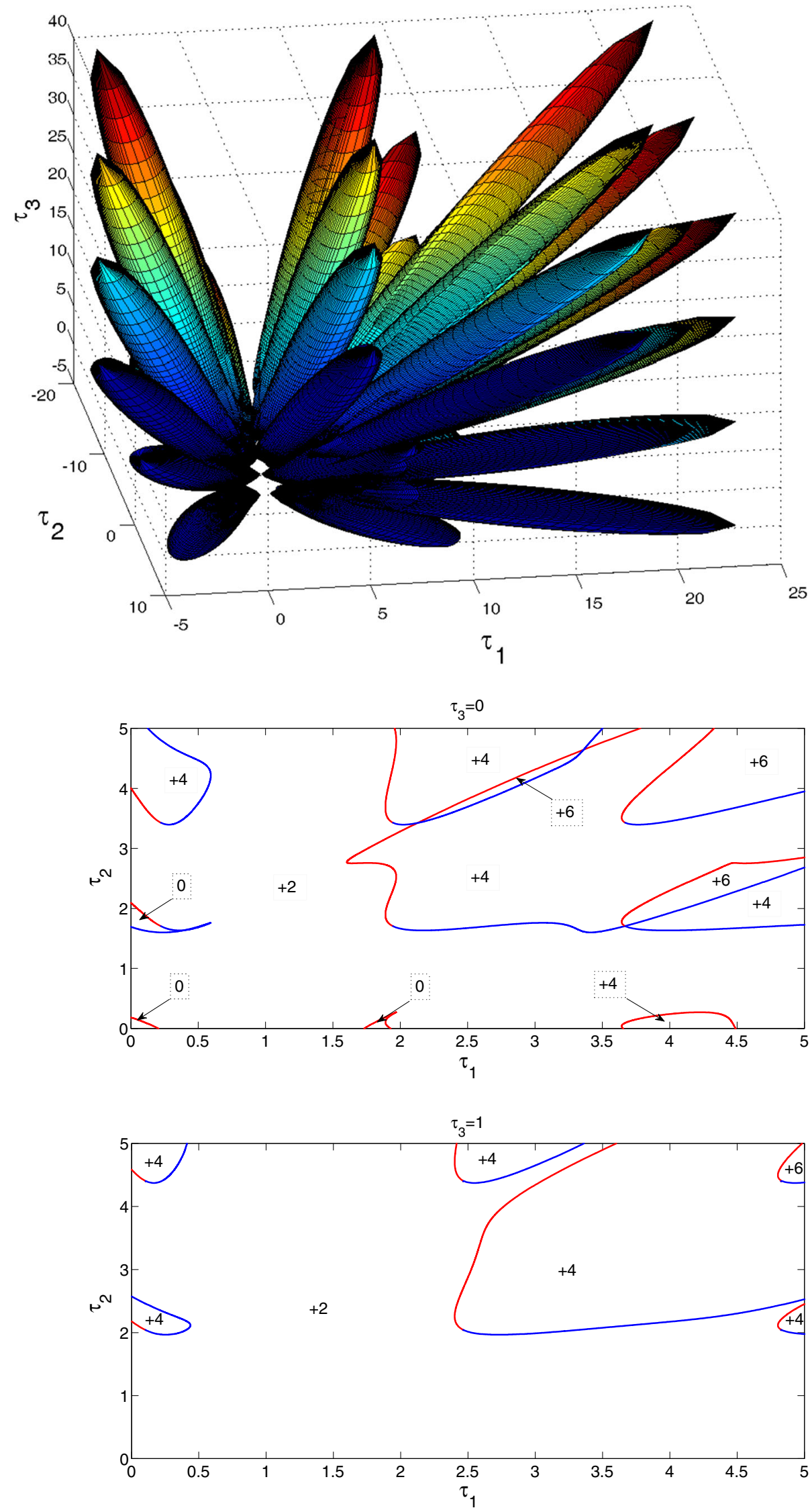
that $\Delta(s)$ has two right-half-plane roots when the delays are outside of the cap-pipe-cap combination.

To see the picture more clearly, we may plot out some cross sections. Figures 22 and 23 show the cross section of the stability crossing set with $\tau_{3}=0$ and 1 , respectively. By calculating the crossing direction at certain strategic locations, the number of right-half-plane roots in each region are calculated, and they are shown in the diagrams. It is seen that there are three stable regions for $\tau_{3}=0$. For $\tau_{3}=1$, the system is unstable for all $\tau_{1}-\tau_{2}$ combination.

\section{Conclusions and an open problem}

A general system with three scalar-delay channels has a characteristic function that contains cross terms that involve the sum of different delays in the expoenents. The stability cross set of such a system contains elements, such as pipes and wavy sheets, that are similar to those for systems without cross terms. However, the elements here are much more rich in the following sense: 1 . There are more possible orientations of, for example, the pipes and wavy sheets that are not possible in the systems without cross terms; 2. There is a new type of maximal interval $\Omega_{M}$ that may correspond to two different elements, such as connectorcap.

Up to now, we have not found any system that contains $\Omega_{M}^{l k}$ with $l \neq k$. It is certainly interesting to either find such a system or prove such a system does not exist.

\section{References}

1. Gryazina EN, Polyak BT, Tremba AA (2008) D-decomposition technique state-of-the-art. Automa Remote Control 69(12): 1991-2026

2. Pontryagin LS (1942) On the zeros of some elementary transcendental functions. Izv Akad Nauk SSSR, Ser Mat 6:115-134 (In Russian). English translation (1955) in Am Math Soc Transl (2) 1:95-110

3. Neimark JuI (1949) D-subdivision and spaces of quasipolynomials. Prikl Mat Mech 13:349-380 (In Russian)

4. El'Sgol'ts LE, Norkin SB (1973) Introduction to the theory and application of differential equations with deviating arguments. Academic Press, New York

5. Stépán G (1989) Retarded dynamical systems: stability and characteristic function. Wiley, New York

6. Bellman RE, Cooke KL (1963) Differential difference equations. Academic Press, New York
7. Henry D (1974) Linear autonomous neutral functional differential equations. J Differ Equ 15:106-128

8. Melvin W (1974) Stability properties of functional difference equations. J Math Anal Appl 48:749-763

9. Meinsma G, Fu M, Iwasaki T (1999) Robustness of the stability of feedback systems with respect to small time delays. Syst Control Lett 36:131-134

10. Willems JC (1971) Analysis of feedback systems. MIT Press, Cambridge

11. Palmor ZJ (1980) Stability properties of Smith dead-time compensator controller. Int J Control 32(6):937-949

12. Engelborghs K, Dambrine M, Roose D (2001) Limitations of a class of stabilization methods for delay systems. IEEE Trans Autom Control 46(2):336-339

13. Van Assche V, Dambrine M, Lafay J-F, Richard J-P (1999) Some problems arising in the implementation of distributed-delay control laws. In: Proceedings of 38th IEEE Conference on Decision and Control, Pheonix, AZ 1999, pp. 4668-4672

14. Gu K (2012) A review of some subtleties of practical relevance for time-delay systems of neutral type. ISRN Appl Math 2012:1-46. doi:10.5402/2012/725783 (Article ID 725783, 46 pages)

15. Ruan S, Wei J (2003) On the zeros of transcendental functions with applications to stability of delay differential equations with two delays. Dyn Contin Discr Impuls Syst 10(6):863-874

16. Hale J, Huang W (1993) Global geometry of the stable regions for two delay differential equations. J Math Anal Appl 178(2):344-362

17. Sipahi R, Olgac N (2003) Stability analysis of multiple time delay systems using the direct method. Paper no. 41495, ASME IMECE, Washington DC, Nov 2003

18. Fazelinia H, Sipahi R, Olgac N (2007) Stability analysis of multiple time delayed systems using 'building block' concept. IEEE Trans Autom Control 52(5):799-810

19. Jarlebring E (2009) Critical delays and polynomial eigenvalue problems. J Comput Appl Math 224(1):296-306

20. Sipahi R, Delice II (2009) Extraction of 3D stability switching hypersurfaces of a time-delay system with multiple fixed delays. Automatica 45(6): 1449-1454

21. Gu K, Niculescu S-I, Chen J (2005) On stability of crossing curves for general systems with two delays. J Math Anal Appl 311(1): $231-253$

22. Gu K, Naghnaeian M (2011) Stability crossing set for systems with three delays. IEEE Trans Autom Control 56(1):11-26

23. Naghnaeian M, Gu K (2013) Stability crossing set for systems with two scalar-delay channels. Automatica 49(7):2098-2106

24. Gu K (2010) Stability problem of systems with multiple delay channels. Automatica 46(4):743-51

25. Carvalho LAV (1996) On quadratic Liapunov functional for linear difference equations. Linear Algebr Appl 240:41-64

26. Hale JK (1975) Parametric stability in difference equations. Bollettino U. M. I. (4) 11(Suppl. fasc. 3):209-214

27. Hale JK, Verduyn Lunel SM (2002) Strong stabilization of neutral functional differential equations. IMA J Math Control Inf 19:5-23

28. Guckenheimer J, Holmes P (1983) Nonlinear oscillations, dynamical systems and bifurcations of vector fields. Springer-Verlag, New York

29. Grashof F (1883) Theoretische Maschinenlehre. Verlag L. Voss, Leipzig 